
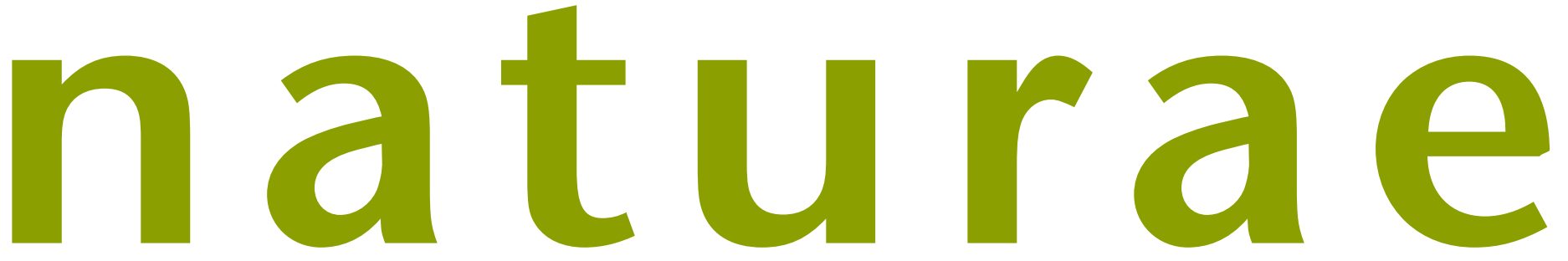

$2019 \cdot 7$

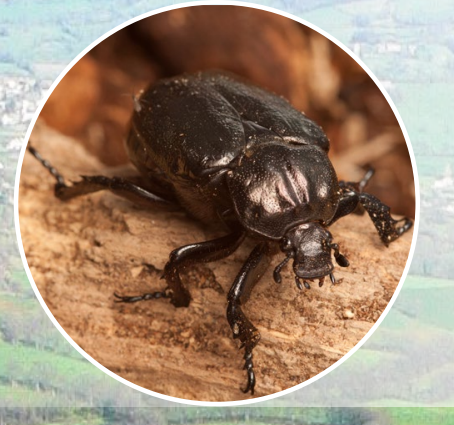

Proposition de protocoles pour la surveillance de l'état de conservation de sept coléoptères saproxyliques de la Directive Habitats-Faune-Flore

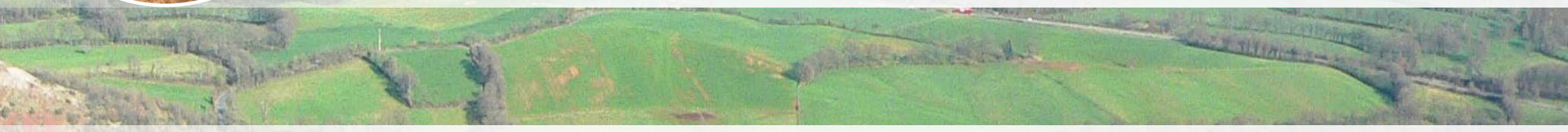

Hervé BRUSTEL, Yoan BRAUD, Nicolas GOUIX, Camille GAZAY, Thierry NOBLECOURT, Lionel VALLADARES, Vincent VIGNON \& Julien TOUROULT

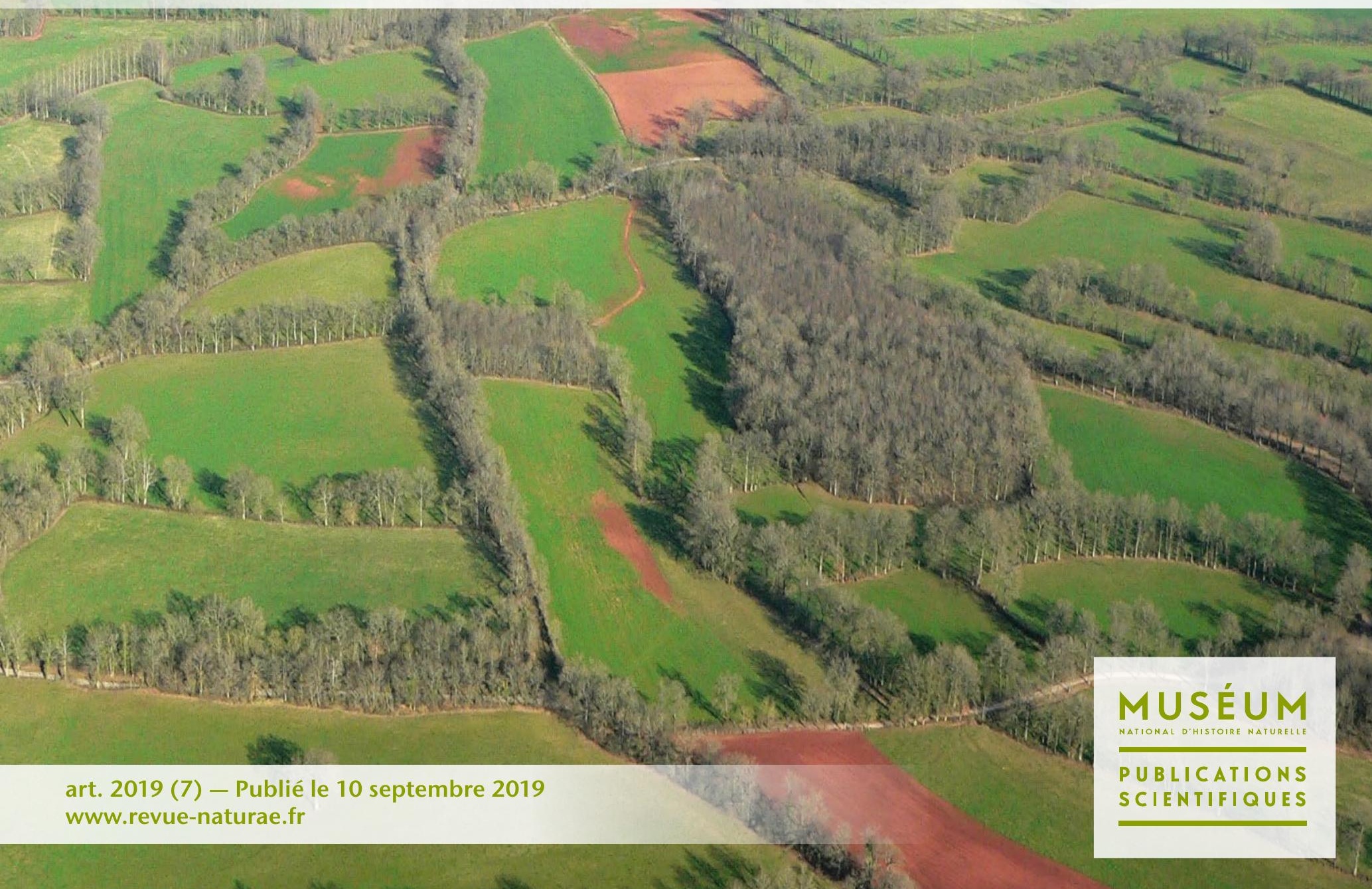


DiRECTEUR DE LA PUbliCATION: Bruno David,

Président du Muséum national d'Histoire naturelle

RÉDACTEUR EN CHEF/EDITOR-IN-CHIEF: Jean-Philippe Siblet

ASSISTANTE DE RÉDACTION/ASSISTANT EDITOR: Sarah Figuet (naturae@mnhn.fr)

Mise en PAge/Page LAyout: Sarah Figuet

COMITÉ SCIENTIFIQUE/SCIENTIFIC BOARD:

Luc Abbadie (UPMC, Paris)

Luc Barbier (Parc naturel régional des caps et marais d'Opale, Colembert)

Aurélien Besnard (CEFE, Montpellier)

Vincent Boullet (Expert indépendant flore/végétation, Frugières-le-Pin)

Hervé Brustel (École d'ingénieurs de Purpan, Toulouse)

Patrick De Wever (MNHN, Paris)

Thierry Dutoit (UMR CNRS IMBE, Avignon)

Éric Feunteun (MNHN, Dinard)

Romain Garrouste (MNHN, Paris)

Grégoire Gautier (DRAAF Occitanie, Toulouse)

Olivier Gilg (Réserves naturelles de France, Dijon)

Frédéric Gosselin (Irstea, Nogent-sur-Vernisson)

Patrick Haffner (UMS PatriNat, Paris)

Frédéric Hendoux (MNHN, Paris)

Xavier Houard (OPIE, Guyancourt)

Isabelle Leviol (MNHN, Concarneau)

Francis Meunier (Conservatoire d'espaces naturels - Picardie, Amiens)

Serge Muller (MNHN, Paris)

Francis Olivereau (DREAL Centre, Orléans)

Laurent Poncet (UMS PatriNat, Paris)

Nicolas Poulet (AFB, Vincennes)

Jean-Philippe Siblet (UMS PatriNat, Paris)

Laurent Tillon (ONF, Paris)

Julien Touroult (UMS PatriNat, Paris)

COUVERTURE/COVER:

Bocage aveyronnais, habitat secondaire de plusieurs coléoptères saproxyliques de la Directive Habitats-Faune-Flore. Crédit photo: L. Valladares.

En médaillon: Osmoderma eremita (Scopoli, 1763). Crédit photo: N. Gouix.

Naturae est une revue en flux continu publiée par les Publications scientifiques du Muséum, Paris

Naturae is a fast track journal published by the Museum Science Press, Paris

Les Publications scientifiques du Muséum publient aussi/The Museum Science Press also publish:

Adansonia, Zoosystema, Anthropozoologica, European Journal of Taxonomy, Geodiversitas, Cryptogamie sous-sections Algologie, Bryologie, Mycologie.

Diffusion - Publications scientifiques Muséum national d'Histoire naturelle

CP $41-57$ rue Cuvier F-75231 Paris cedex 05 (France)

Tél. : 33 (0)1 407948 05/Fax: 33 (0)1 40793840

diff.pub@mnhn.fr/http://sciencepress.mnhn.fr

(C) Publications scientifiques du Muséum national d'Histoire naturelle, Paris, 2019

ISSN (imprimé/print): 1280-9551/ ISSN (électronique/electronic): 1638-9387 


\section{Proposition de protocoles pour la surveillance de l'état de conservation de sept coléoptères saproxyliques de la Directive Habitats-Faune-Flore}

Hervé BRUSTEL

Université de Toulouse, École d'Ingénieurs de Purpan, UMR INRA/INPT 1201 "Dynamique et écologie des paysages agriforestiers ", 75 voie du Toec, boîte postale 57611, F-31076 Toulouse cedex 3 (France) herve.brustel@purpan.fr

Yoan BRAUD

Bureau d'études ENTOMIA, Rue du Moiré de Provence, F-04200 Thèze (France) yoan_braud@yahoo.fr

Nicolas GOUIX

Conservatoire d'Espaces Naturels Midi-Pyrénées, 75 voie du Toec, boîte postale 57611, F-31076 Toulouse cedex 3 (France) nicolas.gouix@espaces-naturels.fr

Camille GAZAY

UMS Patrimoine naturel («PatriNat»), AFB, CNRS, MNHN, 57 rue Cuvier, boîte postale 41, F-75231 Paris cedex 05 (France) gazay@mnhn.fr

Thierry NOBLECOURT Laboratoire national d'entomologie forestière de l'ONF, Pôle National d'Entomologie Forestière, Laboratoire partagé OPIE-ONF, 2 rue Charles Péguy, F-11500 Quillan (France) thierry.noblecourt@onf.fr

Lionel VALLADARES

Université de Toulouse, École d'Ingénieurs de Purpan, UMR INRA/INPT 1201 "Dynamique et écologie des paysages agriforestiers", 75 voie du Toec, boîte postale 57611, F-31076 Toulouse cedex 3 (France) lionel.valladares@purpan.fr

Vincent VIGNON

Office de Génie Écologique, 5 boulevard de Créteil, F-94100 Saint-Maur-des-Fossés (France) v.vignon@oge.fr

Julien TOUROULT

UMS Patrimoine naturel («PatriNat»), AFB, CNRS, MNHN, 57 rue Cuvier, boîte postale 41, F-75231 Paris cedex 05 (France) touroult@mnhn.fr

Soumis le 12 février 2019 | Accepté le 7 juin 2019 | Publié le 10 septembre 2019

Brustel H., Braud Y., Gouix N., Gazay C., Noblecourt T., Valladares L., Vignon V. \& Touroult J. 2019. - Proposition de protocoles pour la surveillance de l'état de conservation de sept coléoptères saproxyliques de la Directive HabitatsFaune-Flore. Naturae 2019 (7): 175-210. https://doi.org/10.5852/naturae2019a7 


\section{MOTS CLÉS}

Forêt,

Natura 2000 sciences participatives, espèces d'intérêt communautaire, insectes protégés.

KEY WORDS

Forest,

Natura 2000,

citizen science,

European community

species of interest,

protected insects.

\section{RÉSUMÉ}

La Directive Habitats-Faune-Flore (DHFF) prévoit un suivi dans le temps de l'état de conservation des espèces d'intérêt communautaire. En France, cette surveillance reste peu développée pour les invertébrés. Des protocoles nationaux sont proposés pour sept espèces de coléoptères saproxyliques rares ou à caractère "parapluie": Stephanopachys linearis (Kugelann, 1792) et Stephanopachys substriatus (Paykull, 1800) (Bostrichidae), Cerambyx cerdo Linnaeus, 1758 et Rosalia alpina (Linnaeus, 1758) (Cerambycidae), Osmoderma eremita (Scopoli, 1763) (Trichiinae), Limoniscus violaceus (P.W.J. Müller,1821) (Elateridae) et Rhysodes sulcatus (Fabricius, 1787) (Rhysodinae). La DHFF définit quatre paramètres d'état de conservation: l'aire de répartition, la taille des populations, l'habitat d'espèce et les perspectives futures. À partir de la littérature sur l'écologie et la conservation de ces espèces et de l'expertise des auteurs sur les populations françaises, la réflexion a porté sur des protocoles économiquement et techniquement réalistes pour effectuer une veille à large échelle. Pour ces espèces rares et discrètes, les mesures directes des tailles de populations ne paraissent pas opérationnelles. Il est proposé de confirmer l'occupation des sites (voire de détecter de nouvelles localités) et de suivre les micro-habitats clés de chacune de ces espèces. Ce focus sur l'habitat d'espèce doit permettre de faire le lien avec les pressions et les mesures de gestion. Un recours à la modélisation et aux observatoires participatifs est envisagé pour préciser la distribution de certaines espèces. Il est préconisé de mobiliser des statistiques de l'inventaire forestier national en les affinant à l'échelle de la distribution de chaque espèce. Un tableau résume les propositions pour chaque espèce: paramètres visés, plan et unités d'échantillonnage, techniques et matériel, périodes favorables, possibilité d'impliquer les gestionnaires, périodicité des relevés, types d'indicateurs, éléments de coûts et perspectives de recherche. Ces propositions sont discutées en termes de moyens nécessaires et au regard des rares protocoles disponibles dans les pays concernés par ces espèces, notamment en Italie.

\begin{abstract}
Monitoring protocols for the conservation status of seven French saproxylic beetles of the European Habitats-Fauna-Flora Directive.

The Habitats Directive requires the conservation status of species of Community interest to be monitored over time. In France, this monitoring remains poorly developed for invertebrates. National protocols are proposed for seven species of saproxylic beetles that are rare or umbrella species: Stephanopachys linearis (Kugelann, 1792) and Stephanopachys substriatus (Paykull, 1800) (Bostrichidae), Cerambyx cerdo Linnaeus, 1758 and Rosalia alpina (Linnaeus, 1758) (Cerambycidae), Osmoderma eremita (Scopoli, 1763) (Trichiinae), Limoniscus violaceus (P. W. J. Müller, 1821) (Elateridae) and Rhysodes sulcatus (Fabricius, 1787) (Rhysodinae). The Habitats Directive defines four parameters of conservation status: range, population size, species habitat and future prospects. Based on the literature on the ecology and conservation of these species and the authors' expertise on French populations, the study focuses on economically and technically realistic protocols for monitoring on a large scale. For these rare and inconspicuous species, direct measurements of population sizes do not appear to be operational. It is proposed to confirm the occupancy of the sites (or even to detect new localities) and to monitor the key micro-habitats of each of these species. This focus on species habitat should make it possible to make the link with pressures and management measures. The use of modelling and participatory observatories is being considered to clarify the distribution of certain species. It is recommended to mobilize statistics from the national forest inventory by refining them at the scale of the distribution of each species. A table summarizes the proposals for each species: target parameters, sampling plan and units, techniques and equipment, favourable periods, possibility of involving managers, frequency of surveys, types of indicators, cost elements and research perspectives. These proposals are discussed in terms of the necessary resources and in the light of the few protocols available in the countries concerned by these species, particularly in Italy.
\end{abstract}

\section{INTRODUCTION}

Depuis 1992 et en complément de la directive Oiseaux de 1979 (2009/147/EC), la directive Habitats-Faune-Flore (92/43/EEC, citée comme DHFF dans la suite de l'article) fonde le socle réglementaire pour la conservation de la nature au sein de l'Union européenne (citée «UE» par la suite). Cette directive définit une liste d'habitats naturels et semi-naturels (Annexe I) et d'espèces (Annexe II) d'intérêt communautaire «dont la conservation nécessite la désignation de zones spéciales de conservation" afin de constituer un réseau de sites: le réseau "Natura 2000 ». Le dispositif est complété par la protection stricte des espèces listées à l'annexe IV et de leur habitat d'espèce et l'annexe $V$ liste d'espèces dont les prélèvements dans la nature et l'exploitation peuvent faire l'objet de mesure de gestion. 
TABLEAU 1. - Coléoptères saproxyliques présents en France. Les espèces surlignées sont celles traitées dans cet article.

\begin{tabular}{|c|c|c|c|c|c|}
\hline $\begin{array}{l}\text { Famille } \\
\text { ou sous-famille }\end{array}$ & Espèce & Nom vernaculaire & Annexe II & Annexe IV & $\begin{array}{r}\text { Protection } \\
\text { nationale }\end{array}$ \\
\hline \multirow[t]{2}{*}{ Bostrichidae } & Stephanopachys linearis (Kugelann, 1792) & & $\mathrm{x}$ & & \\
\hline & Stephanopachys substriatus (Paykull, 1800) & & $\mathrm{x}$ & & \\
\hline \multirow[t]{2}{*}{ Cerambycidae } & Cerambyx cerdo Linnaeus, 1758 & Grand Capricorne du chêne & $\mathrm{x}$ & $\mathrm{x}$ & $\mathrm{x}$ \\
\hline & Rosalia alpina (Linnaeus, 1758) & Rosalie des Alpes & $\mathrm{x}$ & $\mathrm{x}$ & $\mathrm{x}$ \\
\hline Trichiinae & Osmoderma eremita (Scopoli, 1763) & $\begin{array}{l}\text { Pique-prune } \\
\text { ou Osmoderme }\end{array}$ & $x$ & $x$ & $x$ \\
\hline Cucujidae & Cucujus cinnaberinus (Scopoli, 1774) & & $\mathrm{x}$ & $\mathrm{x}$ & $\mathrm{x}$ \\
\hline Elateridae & Limoniscus violaceus (P.W J. Müller, 1821) & Taupin violacé & $\mathrm{x}$ & & \\
\hline Lucanidae & Lucanus cervus (Linnaeus, 1758 ) & Lucane cerf-volant & $\hat{x}$ & & \\
\hline Melandryidae & Phryganophilus ruficollis (Fabricius, 1798) & & $\mathrm{x}$ & $\mathrm{x}$ & $\mathrm{x}$ \\
\hline Rhysodinae & Rhysodes sulcatus (Fabricius, 1787) & & $\mathrm{x}$ & & \\
\hline
\end{tabular}

\section{LES ENJEUX FRANÇAIS DES COLÉOPTÈRES SAPROXYLIQUES} DE LA DHFF

Concernant les insectes, ces listes ont fait l'objet de nombreuses critiques (Cardoso 2012) car la proportion d'insectes y est faible et que beaucoup d'espèces à forts enjeux de conservation n'y figurent pas tandis que d'autres considérées comme à faibles enjeux y figurent. Pour les coléoptères saproxyliques français, la liste s'avère restreinte mais comprend quelques espèces réellement rares et exigeantes, ainsi que des espèces plus communes mais pouvant néanmoins constituer de bons "outils» pour la conservation (Brustel et al. 2013), notamment par leur prise en compte et leur gestion dans le réseau Natura 2000.

Les coléoptères saproxyliques, représentés par seulement 10 espèces en France au sein de ces annexes (Tableau 1), ont une importance fondamentale dans la constitution et la qualité du réseau de sites Natura 2000 boisés mis en place. L'enjeu de conservation qu'ils représentent fut mis en avant dès la fin des années 80 auprès de la Commission européenne (Speight 1989). En France, leur prise en considération s'est amplifiée au début des années 2000 avec des travaux fondateurs au niveau national, notamment sur leur rôle en tant qu'indicateur biologique de la qualité des forêts (Bouget 2004 ; Brustel 2002). L'intérêt porté envers ces quelques espèces s'explique par ce qu'elles représentent: elles témoignent d'attributs de maturité théoriquement inféodés aux forêts les plus anciennes et à dynamique sub-naturelle devenues très rares (Cateau et al. 2015). Quelques espèces ont colonisé au cours du temps des habitats de substitution: arbres taillés des bocages et arbres greffés de verger (Vignon 2005), comme par exemple le Pique-prune, Osmoderma eremita (Scopoli, 1763) qui se trouve aujourd'hui quasi exclusivement dans les milieux agro-pastoraux. Elles restent cependant de formidables espèces parapluie dépendantes de l'évolution ultime d'un dendro-microhabitat (Larrieu 2014; Larrieu et al. 2018) issu de processus séculaires (Ranius et al. 2009).

Parmi les espèces présentes dans les annexes de la DHFF (Tableau 1), toutes n'ont pas le même niveau d'intérêt pour l'identification et la gestion d'un réseau de forêts ou bocages remarquables à l'échelle nationale.
Certaines d'entre elles sont si rares voire incertaines en France qu'elles ne sont pas représentatives d'une situation nationale. C'est notamment le cas de Cucujus cinnaberinus (Scopoli, 1774), espèce en forte recolonisation en Europe, découverte récemment en France dans trois localités alsaciennes au bord du Rhin (Fuchs et al. 2014) ou de Phryganophilus ruficollis (Fabricius, 1798) connu par une seule donnée historique dans la Drôme (Brustel \& Gouix 2012).

À l'inverse, le Lucane cerf-volant Lucanus cervus (Linnaeus, 1758) apparait trop largement distribué et peu exigeant sur le territoire national pour être discriminant dans une stratégie de création d'un réseau de sites à préserver. Cette espèce cependant reste intéressante comme outil de sensibilisation (espèce "porte drapeau") et pour encourager au maintien de bois mort dans toutes sortes de contextes. Elle est déjà étudiée dans le cadre d'une enquête participative organisée par l'Opie (Rabinovitch et al. 2017) et nous ne développerons pas de recommandations sur cette espèce, car elle relève d'une autre logique de surveillance que celles des espèces « rares».

Pour ces raisons, notre travail se concentre sur les espèces «outils» qui représentent un enjeu national en termes de préservation et qui, jusqu'à présent, n'ont pas réellement fait l'objet de programmes nationaux de suivi. Dans le cadre d'une stratégie nationale où l'effort de suivi doit être optimisé (Tableau 2), on distinguera trois types d'enjeux pour justifier le suivi:

- les espèces "parapluies" présentant un fort enjeu de conservation pour les forêts feuillues et le patrimoine arboré à forte maturité des plaines et collines: Osmoderma eremita et Limoniscus violaceus (P. W. J. Müller, 1821); et pour les forêts de montagne: Rhysodes sulcatus (Fabricius, 1787). Ces espèces patrimoniales concernent généralement de faibles surfaces (mise à part de rares agrosystèmes qui peuvent couvrir une centaine d'hectares ou davantage) et quelques sites exceptionnels; - les espèces témoins d'une dynamique de fonctionnement de l'écosystème qui ne sont pas forcément inféodées aux milieux les plus matures: les Stephanopachys spp.;

- les espèces relativement communes sur une partie du territoire national représentatives de presque tous les types de forêts feuillues matures (de plaine, dont ripisylves, de collines et de montagnes) : le Grand Capricorne, Cerambyx cerdo Linnaeus, 1758 et la Rosalie des Alpes, Rosalia alpina (Linnaeus, 1758). 
TABLEAU 2. - Sélection des espèces dont la détection et le suivi sont les plus importantes pour la conservation des cortèges saproxyliques (espèces parapluies). Les espèces surlignées sont celles étudiées dans cet article.

\begin{tabular}{|c|c|c|c|c|}
\hline Taxons & $\begin{array}{l}\text { Connaissances (Biologie/ } \\
\text { Distribution) }\end{array}$ & $\begin{array}{l}\text { Enjeux de conservation: } \\
\text { espèce }\end{array}$ & $\begin{array}{l}\text { Enjeux de conservation: } \\
\text { sites \& milieux }\end{array}$ & Références clés \\
\hline $\begin{array}{l}\text { Limoniscus violaceus } \\
\text { (P.W.J. Müller, 1821) } \\
\text { (Elateridae) }\end{array}$ & Assez bonne/Moyenne & $\begin{array}{l}\text { Toute la France; espèce } \\
\text { rare et parapluie (cavités } \\
\text { basses). }\end{array}$ & $\begin{array}{l}\text { Forêts feuillues de plaine } \\
\text { (chênaies, hêtraies); } \\
\text { bocages. }\end{array}$ & $\begin{array}{l}\text { Gouix } 2011 \\
\text { Gouix et al. } 2015\end{array}$ \\
\hline $\begin{array}{l}\text { Osmoderma eremita } \\
\text { (Scopoli, 1763) } \\
\text { (Trichiinae) }\end{array}$ & Bonne/très bonne & $\begin{array}{l}\text { Espèce rare et parapluie } \\
\text { (vieux arbres à grosses } \\
\text { cavités). }\end{array}$ & $\begin{array}{l}\text { Forêts feuillues de plaine } \\
\text { et moyennes montagnes; } \\
\text { bocages; arbres hors } \\
\text { forêts. }\end{array}$ & Dubois 2009 \\
\hline $\begin{array}{l}\text { Rhysodes sulcatus } \\
\text { (Fabricius, 1787) } \\
\text { (Rhysodinae) }\end{array}$ & $\begin{array}{l}\text { Faible/Assez bonne (des } \\
\text { re-découvertes possibles) }\end{array}$ & $\begin{array}{l}\text { Espèce rare, connue des } \\
\text { Pyrénées du Cantal et } \\
\text { de Lozère et parapluie } \\
\text { (continuité et volume de } \\
\text { gros bois au sol). }\end{array}$ & $\begin{array}{l}\text { Forêts résineuses de } \\
\text { montagne (hêtraies } \\
\text { sapinières). }\end{array}$ & $\begin{array}{l}\text { Brustel \& Gouix } 2011 \\
\text { Kostanjsek et al. } 2018\end{array}$ \\
\hline $\begin{array}{l}\text { Stephanopachys spp. } \\
\text { (Bostrichidae) }\end{array}$ & Faible/Faible & $\begin{array}{l}\text { Espèces méconnues et } \\
\text { localisées (montagnes } \\
\text { méridionales), pionnières, } \\
\text { parapluies restreints } \\
\text { aux dynamiques de } \\
\text { perturbations. }\end{array}$ & $\begin{array}{l}\text { Mélézin; forêts résineuses } \\
\text { de montagne. }\end{array}$ & $\begin{array}{l}\text { Brustel et al. } 2013 \\
\text { Braud et al. } 2017\end{array}$ \\
\hline $\begin{array}{l}\text { Cerambyx cerdo } \\
\qquad \text { Linnaeus, } 1758 \\
\text { (Cerambycidae) }\end{array}$ & $\begin{array}{l}\text { Bonne/moyenne dans le Sud } \\
\text { où elle est répandue, bonne } \\
\text { dans le Nord }\end{array}$ & $\begin{array}{l}\text { Espèce assez commune } \\
\text { voire commune mais } \\
\text { parapluie (vieux arbres, } \\
\text { espèce ingénieur qui } \\
\text { favorise l'installation } \\
\text { d'autres saproxyliques). }\end{array}$ & $\begin{array}{l}\text { Chênaies de plaine et } \\
\text { moyennes montagnes; } \\
\text { Chênes isolés et en } \\
\text { alignements. }\end{array}$ & Buse et al. 2008 \\
\hline $\begin{array}{l}\text { Rosalia alpina } \\
\text { (Linnaeus, 1758) } \\
\text { (Cerambycidae) }\end{array}$ & Bonne/Bonne & $\begin{array}{l}\text { Espèce discrète à } \\
\text { distribution discontinue, } \\
\text { peu parapluie (assez peu } \\
\text { exigeante, milieux variés). }\end{array}$ & $\begin{array}{l}\text { Boisements variés feuillus: } \\
\text { hêtraies, ripisylves, } \\
\text { bocages. }\end{array}$ & $\begin{array}{l}\text { Villiers } 1978 \\
\text { Berger } 2012 \\
\text { Lachat et al. } 2013\end{array}$ \\
\hline $\begin{array}{l}\text { Lucanus cervus } \\
\text { (Linnaeus, 1758) } \\
\text { (Lucanidae) }\end{array}$ & Bonne/Très bonne & $\begin{array}{l}\text { Espèce commune, toute } \\
\text { la France, non parapluie } \\
\text { (peu exigeante, partout) } \\
\text { pour la désignation de } \\
\text { sites, mais utile pour la } \\
\text { prise en compte de la } \\
\text { biodiversité saproxylique } \\
\text { «ordinaire» dans la } \\
\text { gestion. }\end{array}$ & $\begin{array}{l}\text { Tous types de boisements } \\
\text { feuillus. }\end{array}$ & $\begin{array}{l}\text { Rabinovitch et al. } 2017 \\
\text { Bardiani et al. } 2017 \\
\text { Harvey et al. } 2011\end{array}$ \\
\hline $\begin{array}{l}\text { Cucujus cinnaberinus } \\
\text { (Scopoli, 1763) } \\
\text { (Cucujidae) }\end{array}$ & Bonne/Assez bonne? & $\begin{array}{l}\text { En expansion, récemment } \\
\text { découverte en Alsace, } \\
\text { non parapluie (assez } \\
\text { peu exigeante et trop } \\
\text { localisée). }\end{array}$ & $\begin{array}{l}\text { Essences variées, } \\
\text { ripisylves et forêts } \\
\text { humides; y compris } \\
\text { plantations. }\end{array}$ & Fuchs et al. 2014 \\
\hline $\begin{array}{l}\text { Phryganophilus } \\
\text { ruficollis } \\
\text { (Fabricius, 1798) } \\
\text { (Melandryidae) } \\
\end{array}$ & Très faible/Très faible & $\begin{array}{l}\text { Cryptique, une seule donnée } \\
\text { ancienne en France, non } \\
\text { parapluie (absence de } \\
\text { données, trop localisée). }\end{array}$ & & Brustel \& Gouix 2012 \\
\hline
\end{tabular}

\section{LES PARAMÈTRES D'ÉVALUATION DE L'ÉTAT \\ DE CONSERVATION}

Dans le cadre de la DHFF, en plus des actions de conservation qu'ils doivent mettre en œuvre, les États membres s'engagent à évaluer régulièrement les statuts et les tendances des espèces et types d'habitats pour les rapporter à la Commission européenne.

Au sens de la DHFF, l'état de conservation favorable constitue l'objectif global à atteindre et à maintenir pour tous les types d'habitats et pour les espèces d'intérêt communautaire. Il peut être décrit comme une situation où une espèce prospère (aspects qualitatifs et quantitatifs), où les perspectives quant à la vitalité des populations d'espèce sont favorables et où les éléments écologiques intrinsèques des écosystèmes d'accueil sont propices. De la DHFF (article 17) découle ainsi une obligation de rendre compte tous les six ans de l'état de conservation de chaque espèce d'intérêt communautaire dans chaque région biogéographique (sans se restreindre aux sites Natura 2000). Cet état doit être évalué à partir de quatre grands paramètres (Fig. 1):

- surface et évolution de l'aire de répartition;

- effectifs et tendances de populations;

- surface et qualité de l'habitat d'espèce;

- perspectives futures en tenant compte des pressions et menaces (Evans \& Arvela 2011). 
Paramètres « espèce »

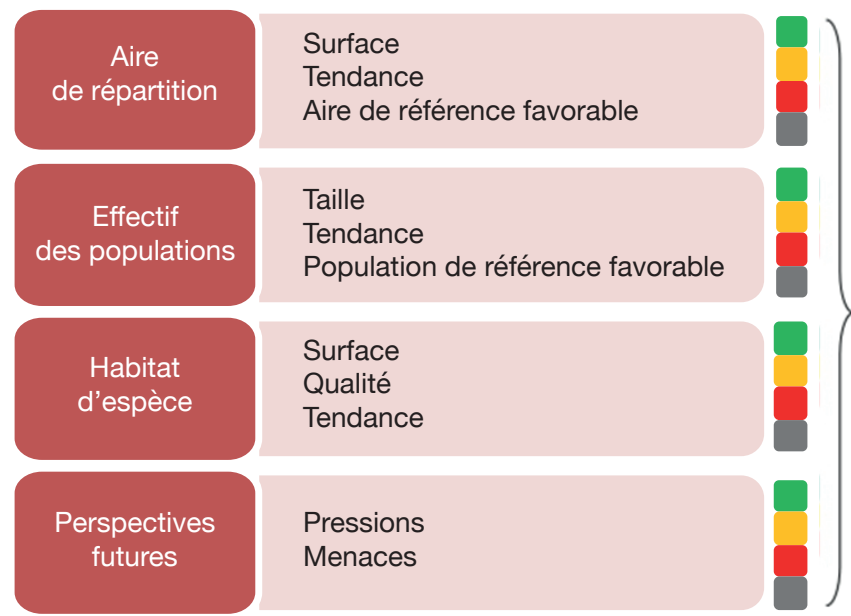

Évaluation

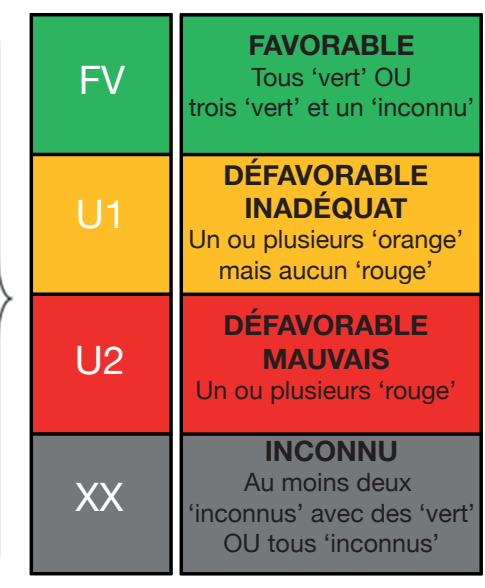

FIG. 1. - Les quatre paramètres d'évaluation d'état de conservation des espèces et de la méthode pour arriver au résultat final dans le cadre de l'évaluation communautaire. Ces paramètres s'appuient sur des indicateurs, eux-mêmes renseignés à partir des données issues théoriquement d'une surveillance par région biogéographique (Bensettiti \& Puissauve 2015).

Moins connu que cette obligation de rapportage, l'article 11 de la DHFF impose aussi à chaque État membre de l'UE de "surveiller» l'état de conservation des espèces; c'est-à-dire de suivre dans le temps des indicateurs traduisant l'évolution des différents paramètres. Cette surveillance doit servir notamment à alimenter l'évaluation de l'état de conservation et à piloter une gestion conservatoire adaptative des espèces où des mesures sont prises pour le maintien de l'état de conservation ou s'il se dégrade. Jusqu'à présent, cette obligation de surveillance a été entreprise de façon variable selon les États membres et sa mise en ouvre varie aussi selon les taxons.

Compte tenu de la taille du territoire métropolitain français, du grand nombre d'espèces et d'habitats présents, il paraît difficilement envisageable d'effectuer une surveillance " exhaustive» des espèces et habitats d'intérêt communautaire sur tout le territoire ou même «simplement" à l'intérieur du réseau Natura 2000. Il convient alors de prioriser un déploiement ou le renforcement de dispositifs de suivi des paramètres d'état de conservation. Il faut en particulier adapter un niveau d'ambition réaliste au regard de l'écologie des espèces, des moyens et techniques disponibles (Puissauve et al. 2017). Notamment, dans le cas d'invertébrés difficiles à détecter et à compter, il faut identifier les paramètres les plus pertinents (rapport information/coût d'acquisition) pour assurer cette surveillance.

La dernière révision du guide méthodologique européen (DG environnement 2017) reconnaît la possibilité d'utiliser d'autres unités que le nombre d'individus pour estimer les «effectifs de population» et leurs évolutions. Après avoir proposé des unités alternatives comme le nombre d'arbres ou de cavités occupées (Evans \& Arvela 2011), l'utilisation du nombre de stations comme unité reflétant les tailles de populations pour tous les Coléoptères est désormais conseillé (DG environnement 2017). Cependant, les autres paramètres restent à surveiller. En particulier pour ces espèces saproxyliques, leur habitat d'espèce (quantité et qualité) présente un caractère intégrateur et permet de faire plus facilement le lien avec les actions de gestion que le nombre d'individus (Chiari et al. 2013b; Puissauve et al. 2017). C'est sur cet aspect que la réflexion a été développée.

\section{ProblémATIQUE}

Pour les coléoptères saproxyliques, il n'y a pratiquement aucune surveillance formelle mise en place à large échelle en France, mis à part des enquêtes participatives et atlas (Houard et al. 2013; De Flores \& Sueur 2015; Puissauve et al. 2017). Cependant, de nombreux travaux ont été réalisés en France, dans le cadre de thèses sur ces espèces (Dubois 2009; Gouix 2011), d'études et de synthèses spécifiques (par exemple Braud et al. 2016; 2017). En Europe, des recherches ont été menées sur ces espèces réglementées (par exemple Chiari et al. 2013b) et des guides pour le suivi viennent d'être publiés (Campanaro et al. 2017a; Carpaneto et al. 2017). Certains de ces travaux mettent en avant les questions d'effectifs de populations, sujet de recherche intéressant mais qui ne nous paraît pas être le plus pertinent pour une surveillance économiquement faisable. Globalement, ces différents travaux de recherche ne vont pas jusqu'à une approche opérationnelle de ce que pourrait être un réseau de suivis et ils nécessitent une contextualisation pour le territoire français. Ce besoin est souligné dans les propositions pour une stratégie de connaissance naturaliste établi par le Muséum national d'Histoire naturelle (MNHN) et l'Agence française pour la Biodiversité (Touroult et al. 2017).

Ainsi, cet article traduit le résultat d'une expertise collective pour établir des protocoles permettant de mesurer des aspects clés de l'état de conservation d'espèces de la DHFF pour l'instant totalement absentes dans les programmes nationaux de suivis de la biodiversité.

Le cadrage méthodologique proposé a pour objectif d'accompagner les entomologistes, les bureaux d'études naturalistes et les gestionnaires pour détecter et suivre ces espèces de façon coordonnée dans le cadre d'un futur programme 
national de surveillance. Ce cadre vise à dynamiser et rendre plus homogène l'acquisition de données notamment en vue de répondre le plus efficacement possible aux besoins d'évaluation à large échelle.

Le but de ce document n'est donc pas de fournir un recueil de toutes les méthodes à mettre en place pour faire un suivi scientifique de l'ensemble des paramètres qui jouent sur les populations de ces espèces. La mise en ouvre de telles études généralisées sur l'ensemble du territoire n'est pas réaliste et risquerait de disperser les efforts rendant ainsi inexploitables les résultats. Le projet Life Italien MIPP (http://lifemipp.eu/ mipp/new/index.jsp, dernière consultation le 25 juin 2019) va plus loin en ce sens et permet à tout opérateur voulant approfondir ce genre d'études de trouver des orientations.

L'objectif est ici de faire des propositions, de manière hiérarchisée et assumée, pour les priorités d'action de connaissance à mettre en œuvre pour assurer un suivi satisfaisant de ces espèces, à l'échelle française, en réponse aux exigences de la DHFF.

\section{MATÉRIEL ET MÉTHODES}

\section{CONSTRUCTION DES PROPOSITIONS}

Les fiches de protocole par espèce qui sont proposées dans la suite de l'article sont issues de l'expérience de terrain des auteurs (inventaires naturalistes et recherche en écologie), de la bibliographie sur l'écologie des espèces et d'une relecture collective critique.

Un tableau type récapitulant les différents aspects d'un protocole a été testé sur une espèce puis généralisé. Il comprend la majorité des sujets importants à définir pour un protocole (Ichter et al. 2014). Le protocole est un plan d'étude détaillé expliquant comment les données doivent être collectées pour répondre à une question scientifique (ici l'évolution dans le temps de paramètres d'état de conservation). Il comporte un plan d'échantillonnage qui définit les règles de sélection des unités étudiées, une ou plusieurs techniques et/ou méthodes à appliquer et des règles complémentaires d'application (par exemple une durée, une fréquence, des conditions météorologiques, etc.).

Les propositions qui sont faites intègrent une logique de rapport "information/coût» raisonnable pour un dispositif de surveillance nationale. Ainsi, les travaux sur les tailles de populations ont été jugés moins efficace (indicateurs directs) que des approches fondées sur des proxy (indicateurs indirects). La qualité (structure, continuité, etc.) et la quantité d'habitat sont considérées comme les principaux facteurs explicatifs de la présence et de l'abondance de ces espèces. C'est par ailleurs sur ces deux facteurs que le gestionnaire agit généralement.

Ne sont donc pas inclus des travaux de suivi qui relèvent de démarches expérimentales et de recherche sur les paramètres démographiques des espèces.

\section{LA DÉMARCHE GÉNÉRALE}

Pour les sept espèces visées, deux paramètres ont été étudiés: la distribution et l'habitat d'espèce.

La distribution, dans une optique d'amélioration des connaissances des stations, est un socle préalable à un suivi dans le temps. Pour certaines des espèces, il reste un fort enjeu à trouver de nouvelles stations. Pour d'autres, l'enjeu est moindre car la majorité des stations est déjà connue (ex: Osmoderma eremita dans une bonne partie de sa distribution), ou parce que les espèces sont plutôt répandues et peuvent faire l'objet d'une démarche par échantillonnage et modélisation (ex: Rosalia alpina et Cerambyx cerdo). Enfin dans certains cas, pour des espèces visibles et reconnaissables in situ comme le Lucane cerf-volant (non traité dans cet article) et la Rosalie des Alpes, la distribution peut être utilement complétée voire établie par des observateurs de type sciences citoyennes (Zapponi et al. 2017). Ceci correspond au paramètre «aire de répartition - surface» de l'évaluation DHFF (Fig. 1).

Au sein de cette distribution, il est important de vérifier périodiquement que les populations restent présentes, via une recherche par échantillonnage (espèces répandues) ou en plein (espèces très localisées). Cette recherche doit intégrer le plus possible une quantification relative de la fréquence de détection, par exemple un taux d'habitats occupés, un taux de contacts par piège ou un temps pour trouver une colonie dans une station. Au sens de l'évaluation DHFF, il s'agit du paramètre "aire de répartition - tendance» et aussi du paramètre "population" pris sous l'angle de la fréquence dans les stations de présence et d'une estimation du nombre d'arbres colonisés.

Dans les stations où l'espèce est connue (un échantillon pour les espèces les plus communes), il est important de suivre la quantité et qualité des arbres hôtes et également, pour les habitats les plus longs à obtenir (grandes cavités), le recrutement et le flux de ce type de micro-habitats dans les stations.

Dans les agrosystèmes, souvent insuffisamment pris en compte, une attention doit être portée à la perte de réseau d'arbres, leur organisation spatiale et le risque de fragmentation des habitats alors que ces arbres ne sont plus renouvelés depuis au moins une trentaine d'années. On pourra se référer aux photographies aériennes de l'IGN depuis les années 1950 qui permettent des évaluations des réseaux de bocages et de vergers couplée à des quantifications sur le terrain (caractérisation des arbres avec description et dénombrement des micro-habitats).

Pour les espèces assez répandues et associées à des types d'habitats forestiers bien définis, il est envisagé de s'appuyer sur des résultats de protocoles dendrométriques déjà mis en place de façon représentative de la forêt française, en particulier l'inventaire forestier national mis en œuvre par l'IGN (voir Paillet 2017 pour un récapitulatif des réseaux de suivi de la biodiversité forestière). Il s'agirait d'une analyse particulière, limitée à l'aire de distribution et aux types d'habitats concernés par chaque espèce, en retenant uniquement certaines variables pertinentes au regard de l'écologie de chaque espèce (par exemple, le bois mort sur pied). Pour les autres espèces et également pour la gestion des sites, des inventaires des arbres favorables et de leurs micro-habitats par les gestionnaires sont préconisés. Au sens de l'évaluation DHFF, ceci correspond au paramètre "habitat d'espèce» dans toute ses composantes et également, pour ce qui est du recrutement des micro-habitats, au paramètre "perspectives futures». 


\section{RÉSULTATS : FICHES PROTOCOLE PAR ESPÈCE}

Les résultats du travail d'expertise sont présentés sous forme d'un texte par espèce, accompagné d'un tableau de synthèse récapitulant de façon standardisée les éléments clés du protocole proposé.

\section{PRÉALABLES}

\section{Aspects réglementaires et déontologiques}

Une partie des espèces visées fait l'objet d'une protection stricte (Tableau 1) et une bonne partie des stations de ces espèces patrimoniales sont localisées dans des aires protégées qui pour certaines réglementent les activités de prospection.

La protection des espèces de la DHFF s'accompagne d'une protection de leur habitat d'espèce. Même s'il n'y a pas manipulation ni prélèvement d'individus, la manipulation de l'habitat (par exemple la fouille d'une cavité suspectée abriter le pique-prune) nécessite de faire une demande d'autorisation à la DREAL pour la perturbation de l'habitat d'espèce.

Quel que soit le statut de protection de l'espèce, le droit de propriété implique de demander l'autorisation d'accès aux propriétaires ou gestionnaire pour accéder aux habitats, prospecter ou poser des pièges (a fortiori pour mettre en place du génie écologique). Cet aspect ne s'applique pas dans le cas particulier de propriétés ouvertes au public et des prospections ne nécessitant pas de toucher «au fond» du terrain et de ses arbres.

Pour la manipulation d'espèces protégées, il sera nécessaire de contacter les services de la DREAL en charge de l'application des articles L.411-1 et L.411-2 du code de l'environnement.

Pour les espèces non protégées, des règles déontologiques simples sont à respecter:

- lors de la prospection des cavités, il convient de remettre soigneusement le terreau en place en ne manipulant qu'une partie limitée du volume de terreau (moins d'un tiers) et en veillant à ne pas mettre le terreau de surface dans les couches inférieures plus humides pour limiter l'effet de la manipulation de la cavité;

- pour la prospection dans les grumes cariées, se limiter à la première observation ou à la recherche jusqu'au tiers du volume de carie a priori favorable afin d'éviter de détruire le milieu (ex: Rhysodes sulcatus).

\section{Accéder, gérer et partager les données}

Pour un bon suivi de ces espèces, pour leur évaluation et pour pouvoir définir des plans d'échantillonnage pertinents, il est nécessaire d'avoir accès aux données précises de présence. Dans le cadre du SINP (Système d'Information sur la Nature et les Paysages) et de sa plate-forme nationale, l'INPN (Inventaire national du Patrimoine naturel), un effort important a été conduit pour le partage de données sur les coléoptères saproxyliques (programme d'inventaire SAPROX [http://saprox. mnhn.fr/, dernière consultation le 25 juin 2019] notamment).

Pour les questions de connaissance et de conservation appliquée, il est important que les gestionnaires et les entomologistes signalent leurs observations dans des bases de données qui échangent les données dans le cadre du SINP (ex: Bases de données naturalistes des Réserves naturelles de France, des Conservatoires d'Espaces naturels, des Parcs nationaux, de l'Office national des Forêts, etc.).

Une bonne pratique consiste à joindre systématiquement une preuve à l'observation. Les systèmes de saisie en ligne sur Smartphone sont aujourd'hui performants et permettent de saisir simplement une observation géolocalisée et d'y associer une photo (ex: Carnat, INPN-Espèces, i-Naturalist).

Les données issues des protocoles proposés dans cet article devraient être structurées dans une base nationale de gestion adaptée aux exigences du protocole pour ensuite être partagées comme donnée publique dans le SINP.

Les distributions des espèces sont disponibles sur l'INPN (https://inpn.mnhn.fr, dernière consultation le 25 juin 2019) et mises à jour en fonction des données transmises par les différents contributeurs.

Les données de structures des habitats et d'inventaires des micro-habitats devraient elles aussi être rendues disponibles de façon publique dans le SINP.

\section{LIMONISCUS VIOLACEUS (TAUPIN VIOLACÉ)}

Parmi les coléoptères de la DHFF, le Taupin violacé Limoniscus violaceus est l'une des espèces les plus discrètes et dont la distribution est probablement la moins bien connue, avec un peu moins de 20 localités signalées sur le territoire national et moins de 200 localités sur toute son aire de répartition (Gouix et al. 2012). Le peu de localités actuelles s'explique aussi par une exigence importante envers son micro-habitat. Limoniscus violaceus se développe dans les cavités situées au pied des arbres feuillus. Les larves vivent deux ans dans le terreau en cours de mélange avec le sol avant de se transformer en imago et de sortir (ou non) de la cavité, s'enfonçant parfois jusqu'à plus d'un mètre de profondeur ou entre les fissures des parois de la cavité. Ces mœurs cryptiques expliquent les difficultés rencontrées par les entomologistes pour détecter l'espèce. La plupart des localités repose sur l'observation d'une larve ou d'un adulte découvert dans le terreau. Les découvertes hors cavités, par observation directe ou par battage, restent exceptionnelles et anecdotiques. Cette espèce représente donc un enjeu majeur en terme de protocole que ce soit pour sa détection ou le suivi de ses populations.

\section{Paramètres visés par les protocoles}

Détection de nouvelles stations et confirmation de présence. Il s'agit de l'espèce DHFF dont le potentiel de nouvelles stations à découvrir est aujourd'hui le plus important. La détection de Limoniscus violaceus représente un défi important du fait de l'absence d'une méthode simple et accessible pour y parvenir. Jusqu'à présent, la découverte de nouvelles localités s'est principalement basée sur la découverte de larve ou d'imago lors de l'examen du terreau de cavités basses. L'usage de pièges spécifiques pour l'étude des cavités (Gouix \& Brustel 2012) a permis depuis quelques années d'optimiser la détection et parfois de découvrir de nouvelles localités.

Les deux techniques aujourd'hui préconisées pour détecter Limoniscus violaceus sont l'examen direct du terreau des cavités et la mise en place de pièges à émergence. 

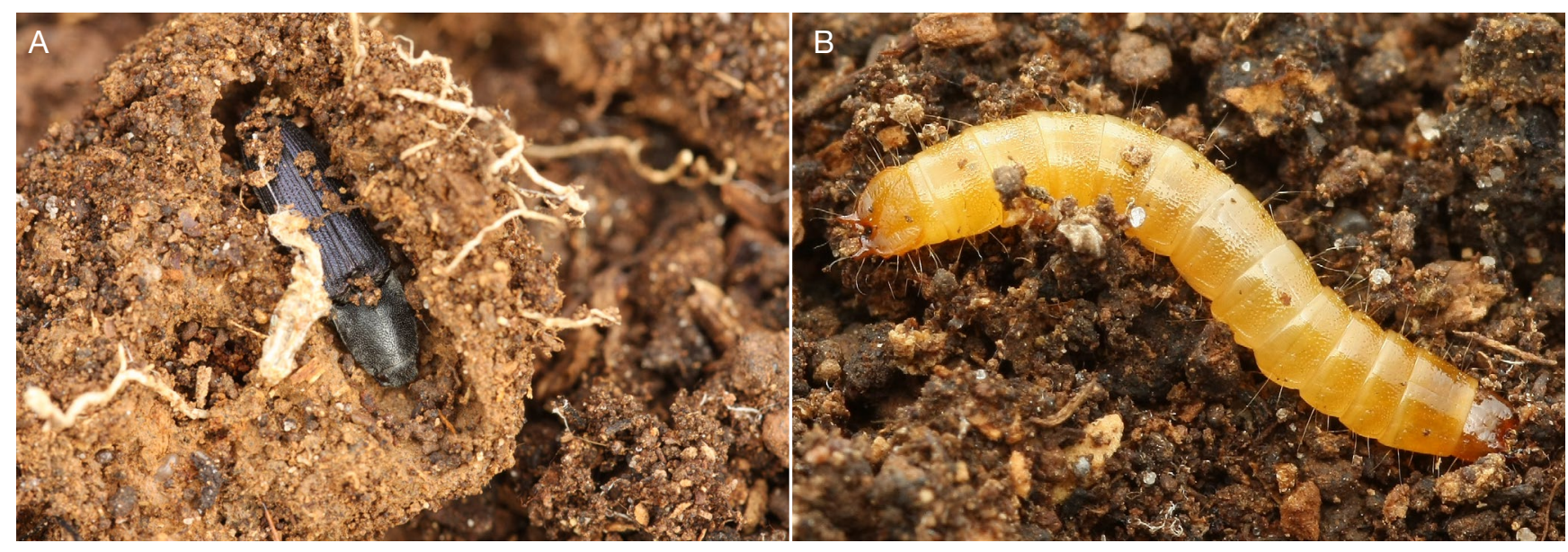

FIG. 2. - Limoniscus violaceus (P.W.J. Müller, 1821): A, imago en loge; B, larve. Crédit photo: N. Gouix.

L'examen du terreau est une méthode efficace pour un entomologiste confirmé et formé à la recherche de l'espèce et si les conditions d'accès au terreau sont favorables. Elle consiste à rechercher minutieusement une larve ou un adulte (Fig. 2) dans le terreau extrait.

Le terreau le plus favorable est caractérisé par sa forte teneur en matière organique et une humidité assez importante qui se traduit par la présence de masse compacte et visqueuse. C'est dans ces petites structures que l'on nomme communément «rognon de terre» ou nodule (Fig. 2) que l'on trouve le plus souvent l'espèce. Dans la plupart des cas, seule une ou quelques larves sont découvertes et parfois un adulte. La détermination de la larve nécessite une bonne expertise et l'utilisation d'une loupe de terrain, cette dernière pouvant être confondue avec des espèces d'Athous spp., notamment haemorrhoidalis (Fabricius, 1801), qui sont des espèces terricoles largement répandues dans les cavités et en dehors (Rudolph 1974). Les adultes, qui se nymphosent à la fin de l'été et passent l'hiver protégés dans leur loge nymphale, présentent peu de risque de confusion, hormis pour des novices avec les espèces Cidnopus pilosus var. cyanescens Buysson, 1902 ou Limonius minutus var. cyanichrous Buysson, 1926 (Luce 1996).

Cette technique de prospection est donc une méthode simple et peu onéreuse par le peu de matériel qu'elle nécessite et offre de bons résultats pour un entomologiste formé. Elle peut être utilisée toute l'année mais les imagos découverts en hiver en loge ne peuvent probablement pas survivre une fois extraits de leur «rognon». Il est donc conseillé de ne pas prospecter les cavités durant la période hivernale. Le début du printemps apparait particulièrement favorable. Les feuilles ne sont pas encore présentes ce qui permet une bonne vision des arbres à cavités. Les imagos commencent à sortir des cavités dès le début du mois d'avril.

Le caractère invasif de la méthode est son principal inconvénient. Un terreau favorable pour le Taupin violacé résulte d'un long processus qui s'étale certainement sur plusieurs décennies. Il en résulte des couches de terreau de différentes textures et compositions selon leur position dans la cavité. L'exploration de ce terreau peut entrainer une déstructura- tion de l'habitat et il est primordial, à la fin de l'examen du terreau, de le redéposer sans mélanger les différentes couches et en recréant de petits rognons (par tassement dans la main d'une poignée de terreau humide) là où ils étaient présents. L'expérience sur une cavité suivie sur plusieurs années nous a montré que le petit rognon recréé était recolonisé les années suivantes.

L'utilisation de pièges à émergence (Gouix \& Brustel 2012) consiste à disposer une toile sur la cavité (Fig. 3A) obligeant les individus qui veulent sortir de la cavité à venir dans un flacon collecteur (Fig. 3B). Ainsi, on peut théoriquement conclure sur la présence ou l'absence de l'espèce dans la cavité à la fin de la période de piégeage. L'autre avantage de cette technique est qu' elle s'adapte à toutes les cavités, même celles avec des entrées trop petites pour une exploration manuelle. La session de piégeage peut être conduite par un gestionnaire mais la détermination des spécimens nécessitera l'intervention d'un entomologiste.

Les conditions d'utilisation optimum de cette technique pour la détection de Limoniscus violaceus ont été étudiées par Gouix (2011). Pour un nouveau site, l'étude d'une vingtaine de cavités entre avril et mai est nécessaire pour pouvoir valider la présence ou l'absence de l'espèce. L'effort d'échantillonnage peut être réduit à cinq cavités si le piégeage s'oriente vers les cavités les plus favorables, c'est-à-dire les cavités fortement évoluées et de très grand diamètre.

Si cette technique apporte des résultats plus robustes que l'examen du terreau, elle présente l'inconvénient d'être létale pour les individus capturés et pour les cortèges des espèces compagnes. Toutefois, si le piège est relevé régulièrement et enlevé dès le premier L. violaceus capturé, on estime que l'impact est négligeable (Gouix 2011). D’un point de vue de la conservation de l'espèce, l'avantage de cette technique est qu'elle n'impacte pas la structuration du terreau dans lequel se développent les larves, en d'autres termes, l'habitat de l'espèce. En revanche, sa mise en ouvre nécessite plus de matériel et de temps disponible avec un suivi régulier tout le long de la période de piégeage (idéalement entre début avril et mi-juin). 

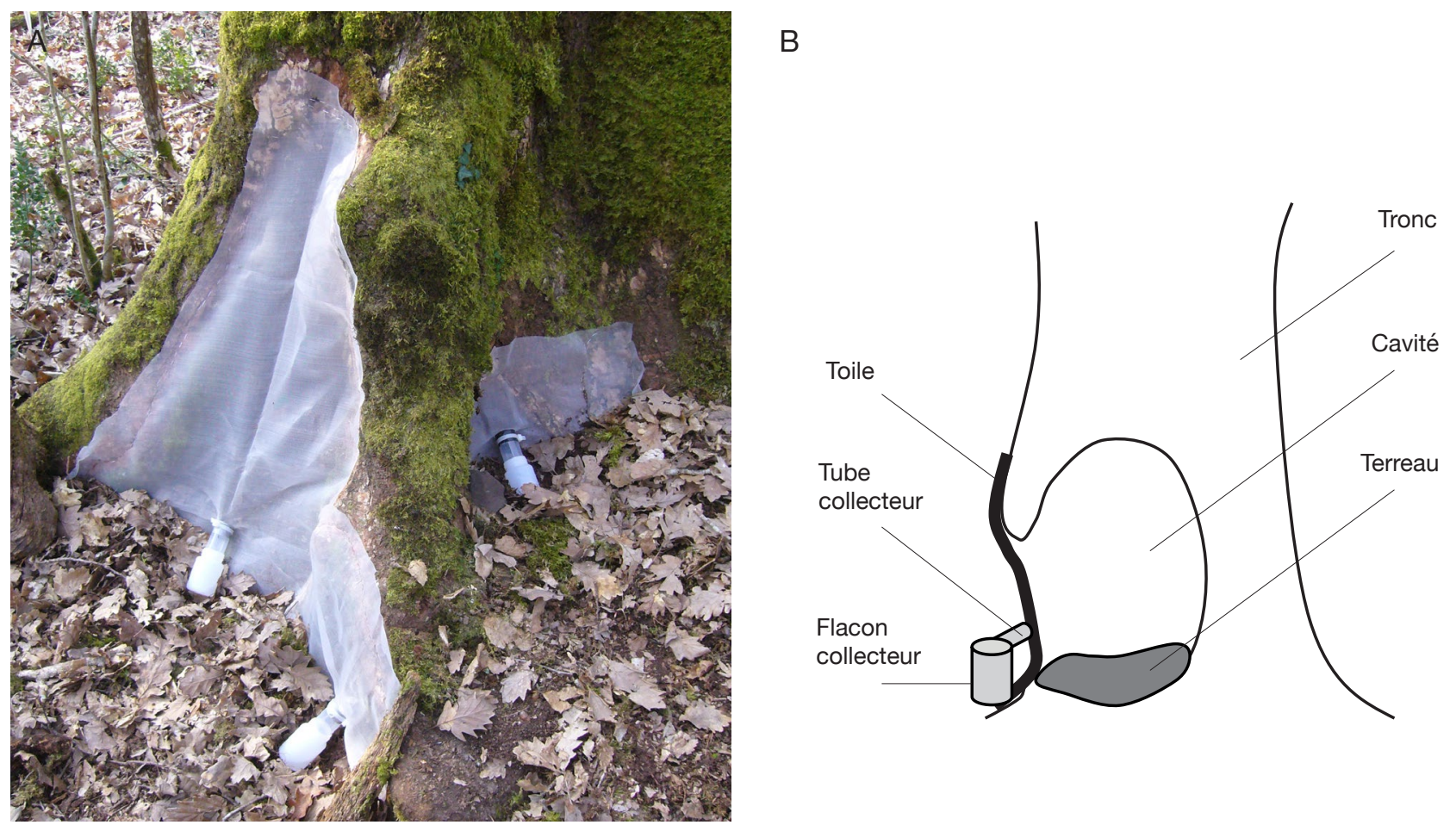

FIG. 3. - A, pièges à émergence adaptés et agrafés autour de cavités basses; $B$, schéma du piège. Crédit photo: N. Gouix

Suivi des habitats. Pour les sites où la présence de L. violaceus est avérée, estimer régulièrement le nombre de cavités occupées par l'espèce de manière certaine est très difficile. Cela nécessiterait d'équiper de pièges à émergence l'ensemble des cavités potentielles, ce qui représente un effort d'échantillonnage conséquent. Dans le cadre d'un suivi, il apparait donc pertinent de mesurer l'évolution de la ressource en micro-habitats favorables au sein d'un site. L'action du gestionnaire concerne la gestion de cette ressource avec la conservation de cavités favorables pour l'espèce et l'anticipation par le maintien de cavités qui deviendront favorables à l'espèce.

Pour ce suivi des habitats, le stade d'avancement de la cavité (Fig. 4) et la circonférence de l'arbre à $30 \mathrm{~cm}$ du sol sont de bons indicateurs des potentialités d'accueil d'une cavité envers Limoniscus violaceus (Gouix et al. 2015).

À partir de ces deux variables a pu être définie une règle de décision qui hiérarchise cette potentialité (Tableau 3). Par exemple, les cavités dont l'état d'avancement est inférieur à 3 et la circonférence de l'arbre à $30 \mathrm{~cm}$ du sol est inférieure à $235 \mathrm{~cm}$ n'hébergent probablement pas Limoniscus violaceus: les enjeux actuels sont faibles. À l'inverse, les cavités dont l'état d'avancement est égal à 5 et la circonférence à $30 \mathrm{~cm}$ du sol est supérieure à $360 \mathrm{~cm}$ doivent être considérées comme hébergeant $L$. violaceus si l'espèce est présente sur le site: les enjeux sont très forts.

Cette règle de décision doit être considérée comme un outil qui oriente le gestionnaire ou l'entomologiste pour évaluer la probabilité qu'une cavité soit favorable à Limoniscus violaceus.

Elle simplifie l'approche de l'habitat d'espèce à la prise en compte de deux variables mais ne permet pas d'expliquer les conditions écologiques de présence du Taupin violacé.
Il est donc important de ne pas considérer ces valeurs comme absolues (Gouix 2011). La méthode doit être évolutive dans le temps et la mise en place d'une stratégie de surveillance nationale doit permettre d'acquérir de nouvelles données amenant à affiner notre modèle.

Pour les sites où l'espèce n'est pas connue, ces informations permettent l'évaluation de la présence d'un habitat qui serait favorable pour l'espèce. Elles peuvent conduire le gestionnaire à envisager la mise en place d'une étude par piégeage à émergence. L'utilisation de cette typologie permet de rationaliser l'effort de piégeage en orientant la mise en place du dispositif sur les cavités les plus favorables. Ainsi, l'étude d'au moins cinq cavités de stade 5 et de circonférence (à $30 \mathrm{~cm}$ du sol) supérieure à $360 \mathrm{~cm}$ permet à plus de $95 \%$ de chance de détecter Limoniscus violaceus si l'espèce est présente.

\section{Orientations pour la mise en place d'une surveillance nationale}

La mise en place d'une surveillance nationale de Limoniscus violaceus poursuit deux objectifs principaux:

- affiner notre connaissance de sa distribution nationale qui passe par la détection de nouveaux sites avérés et des secteurs de présence potentielle, et la confirmation périodique (10 ans) de la présence sur les sites connus;

- suivre les habitats présents et en devenir de l'espèce pour leur prise en considération dans la gestion et pour dégager des tendances d'évolution de l'habitat d'espèces et estimer les perspectives futures.

Pour affiner notre connaissance sur la distribution de l'espèce en France, il est nécessaire d'orienter notre action vers les secteurs favorables à son accueil. Le secteur concerné pour la 


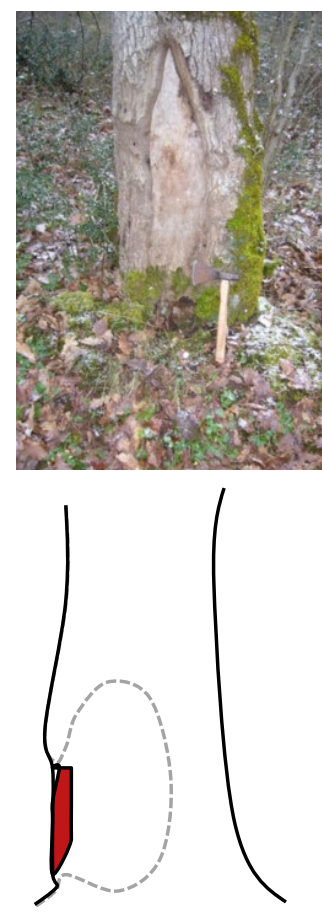

Stade 1

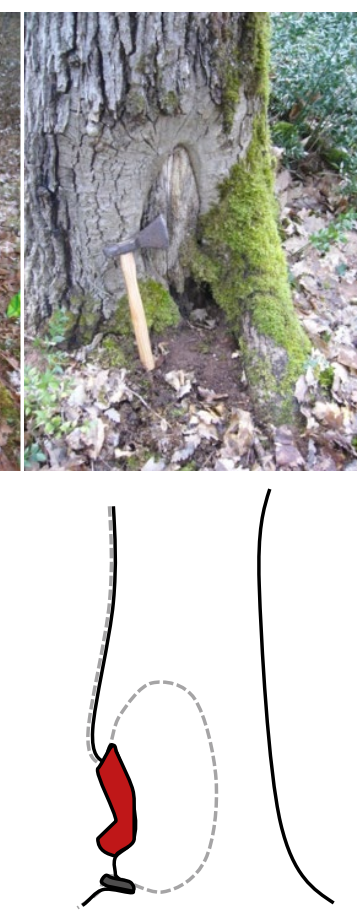

Stade 2

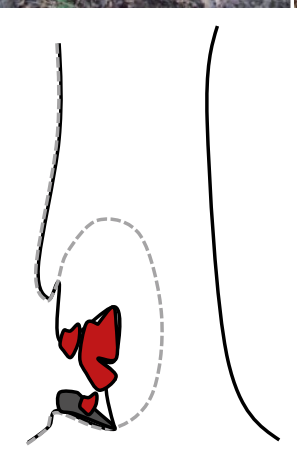

Stade 3
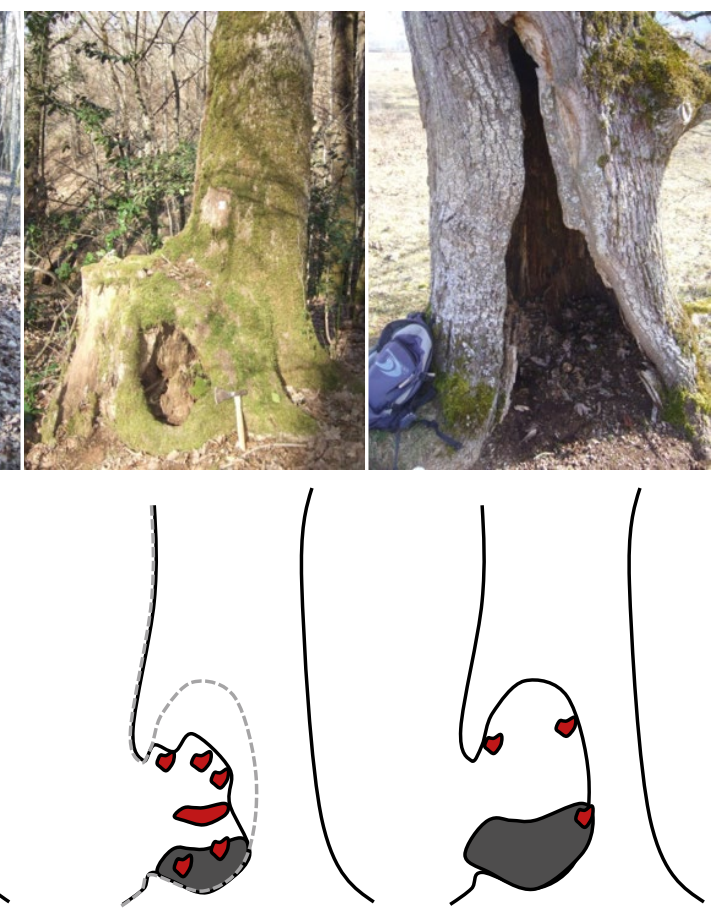

Stade 4

Stade 5

Bois mort en cours de décomposition (carie)

Terreau

FIG. 4. - Stade d'avancement au cours du processus de formation d'une cavité basse, illustré par des photos (en haut) et schématisé (en bas). Crédits photos: N. Gouix.

TABLEAU 3. - Règle de décision pour évaluer la potentialité d'accueil d'une cavité pour Limoniscus violaceus (P. W. J. Müller, 1821) d'un site où il est déjà connu (Gouix 2011). --, présence improbable; -, présence peu probable; + présence probable; ++, présence.

\begin{tabular}{lccc}
\hline \multirow{2}{*}{$\begin{array}{c}\text { Circonférence de l'arbre à } \\
\mathbf{3 0} \mathbf{~ c m} \text { du sol } \mathbf{( c m )}\end{array}$} & \multicolumn{3}{c}{ État d'avancement de la cavité } \\
\cline { 2 - 4 } & $<3$ & 3 ou 4 & 5 \\
\hline 235 & -- & - & - \\
$235-360$ & -- & + & + \\
$>360$ & -- & + & ++ \\
\hline
\end{tabular}

mise en ouvre d'une surveillance nationale concerne toute la France à l'exception des zones d'altitudes au-delà de l'étage montagnard (environ $1500 \mathrm{~m}$ ). L'espèce a été récemment découverte à $850 \mathrm{~m}$ d'altitude dans le Nord de l'Espagne. Elle se développe dans les vieux peuplements feuillus ou les vieux arbres du bocage (Gouix et al. 2012) où sont présentes des cavités basses favorables à l'espèce (Gouix et al. 2015).

La recherche de nouveaux sites par la mise en œuvre d'un protocole de détection doit être priorisée:

- dans les sites où des cavités favorables sont identifiées d'après les remontées de terrains d'entomologistes ou de gestionnaire; - dans les sites où les cortèges d'espèces associées à la présence de Limoniscus violaceus sont connus (Gouix 2011).

Les travaux menés sur les cortèges d'espèces indicateurs de la présence de Limoniscus violaceus (Gouix 2011) permettent de définir différents niveaux de priorité sur les sites à prospecter :
- une priorité de premier ordre sera donnée aux sites hébergeant à la fois Cardiophorus gramineus (Scopoli, 1763) et Ischnodes sanguinicollis (Panzer, 1793), deux autres taupins strictement inféodés aux cavités basses d'arbres. Cette priorité sera renforcée par la présence d'autres espèces plus ou moins cavicoles mais souvent présentes dans les sites occupés par le Taupin violacé: Osmoderma eremita, Megapenthes lugens (W. Redtenbacher, 1842), Lacon querceus (Herbst, 1784), Allecula spp., Podeonius acuticornis (Germar, 1824), Procraerus tibialis (Lacordaire in Boisduval \& Lacordaire, 1835);

- une priorité de second ordre sera donnée aux sites hébergeant au moins Ischnodes sanguinicollis ou Cardiophorus gramineus et une ou plusieurs des espèces citées précédemment. Dans cette seconde priorité, les sites avec Osmoderma eremita souvent caractérisés par la présence de très vieux arbres seront prioritaires; - une priorité de troisième ordre pour les sites hébergeant uniquement Ischnodes sanguinicollis ou Cardiophorus gramineus et pour les sites hébergeant Osmoderma eremita dans lesquelles des cavités basses sont identifiées.

À partir des données d'inventaires sur les saproxyliques disponibles dans l'INPN, les secteurs à prospecter pourront être priorisés et un programme d'étude pluriannuel lancé au niveau national ou en région.

Le suivi des habitats d'espèces concerne tous les sites où l'espèce est avérée (suivi "en plein») compte tenu du faible nombre de tels sites connus. Il constitue en premier lieu un outil pour le gestionnaire devant prendre en considération 


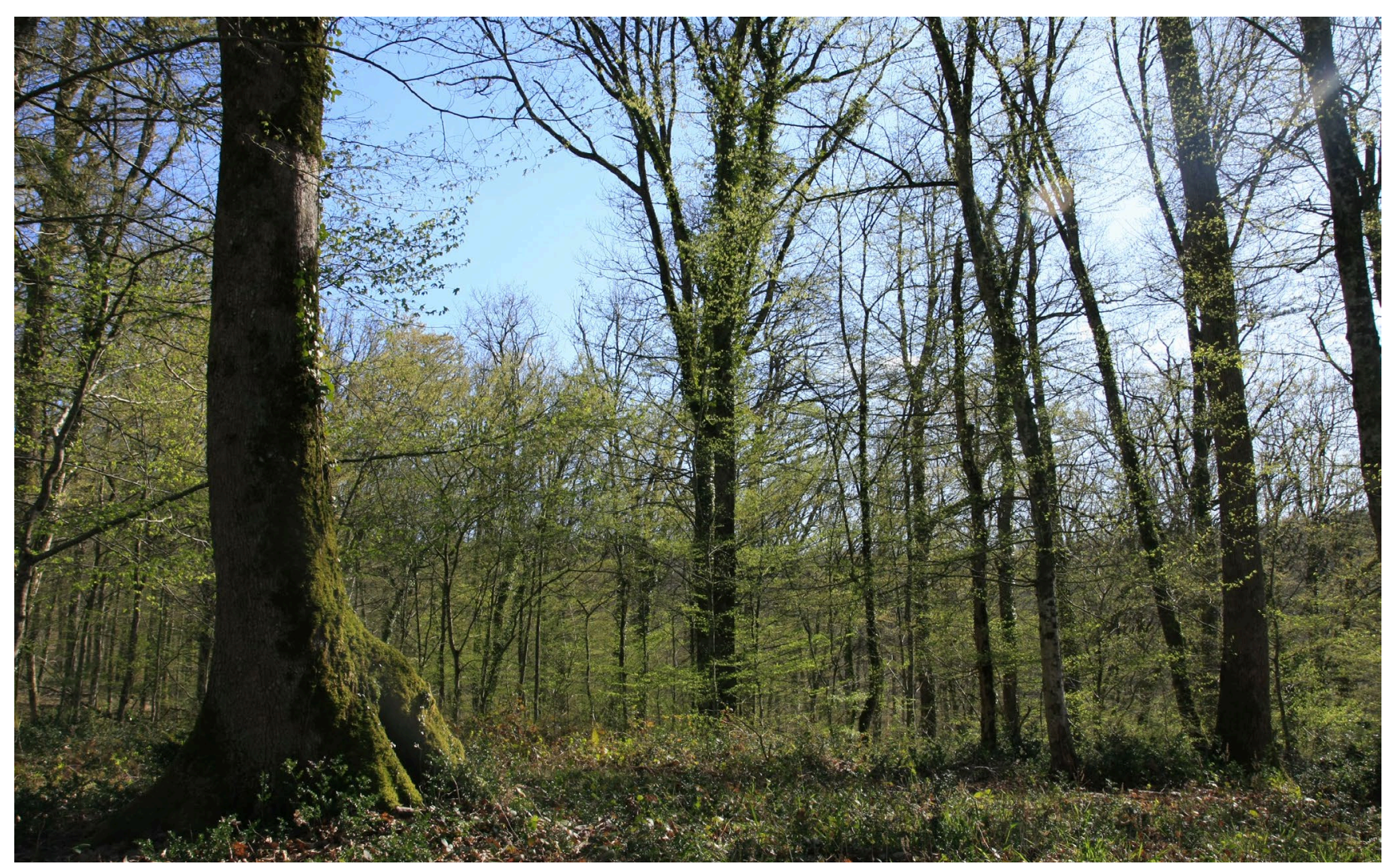

FIG. 5. - Arbre favorable issu d'un taillis recepé et vieilli (jeune futaie sous futaie mature sur souche). Crédit photo: N. Gouix.

Limoniscus violaceus dans la gestion de son site (voir exemple des préconisations de gestion en Grésigne dans Gouix 2011). La capitalisation des données de suivi à l'échelle nationale permettra de fournir des indicateurs pour évaluer les tendances d'évolution de l'habitat d'espèce.

Ce suivi repose sur la caractérisation des cavités à travers deux facteurs simples: circonférence à $30 \mathrm{~cm}$ du sol et stade d'avancement. Il peut être conduit de manière exhaustive sur des sites de petite surface ( $<50$ hectares) ce qui concerne la plupart des localités connues en France. On considèrera un site comme un ensemble d'arbres à cavités favorables situées dans un rayon de $500 \mathrm{~m}$ maximum. Dans des massifs de grande taille comme les forêts de Fontainebleau ou de Tronçais, la présence de l'espèce reste limitée à de petites parcelles qu'il est simple de parcourir sur une ou deux journées. Dans de rares secteurs comme la forêt de Grésigne (Fig. 5) où l'espèce est répartie sur l'ensemble du massif et où les densités de cavités basses peuvent être importantes en lien avec un historique de futaie sur souche, il est nécessaire de mettre en place des placettes d'échantillonnage (permanentes) qui serviront de support au suivi. L'hectare est dans ce cas une unité intéressante par sa facilité de mise en œuvre (placette circulaire de $56 \mathrm{~m}$ environ). Les données acquises dans le cadre de ces suivis permettront d'évaluer l'évolution des densités de cavités favorables à l'espèce entre une situation antérieure et la situation actuelle. Elles permettront également de dégager les tendances d'évolutions futures avec les densités de cavités encore non favorables mais en devenir (densité de cavités aux stades d'avancement 1 et 2, Fig. 4).
Ce travail sur les tendances d'évolution est important pour le gestionnaire qui doit assurer une disponibilité en habitat continue dans le temps sur son site. Il devra ainsi recruter dès les peuplements forestiers les plus jeunes des arbres qui deviendront favorables à l'espèce. Ainsi, dès le stade du perchis, des arbres doivent être conservés dans l'objectif d'obtenir à terme des arbres favorables à Limoniscus violaceus (Gouix 2011). Ces arbres peuvent être des rejets sur souches, ceux-là même qui appelés «loups» sont souvent les premiers à être éliminés par les forestiers dans les conduites en futaie régulière.

Priorités et moyens. La priorité, en raison du faible nombre de localités connues en France est d'affiner la connaissance sur la distribution de l'espèce. La présence de l'espèce dans des arbres isolés des milieux agro-pastoraux indique une potentialité de présence bien plus importante que celle actuellement recensé. Pour comparaison, le Pique-prune dont le niveau d'exigence envers la maturité des cavités d'arbres est comparable est connu dans plus de 300 localités en France. La disparition grandissante de ces vieux arbres en raison des modifications de pratiques agricoles et l'absence de leur renouvellement (Fig. 6) laisse penser que plusieurs populations disparaissent actuellement sans prise en considération car non identifiées préalablement. Les outils proposés hiérarchisent l'organisation des prospections de terrain. Le travail reste considérable pour rattraper un niveau de connaissance similaire à celui acquis depuis ces dernières 


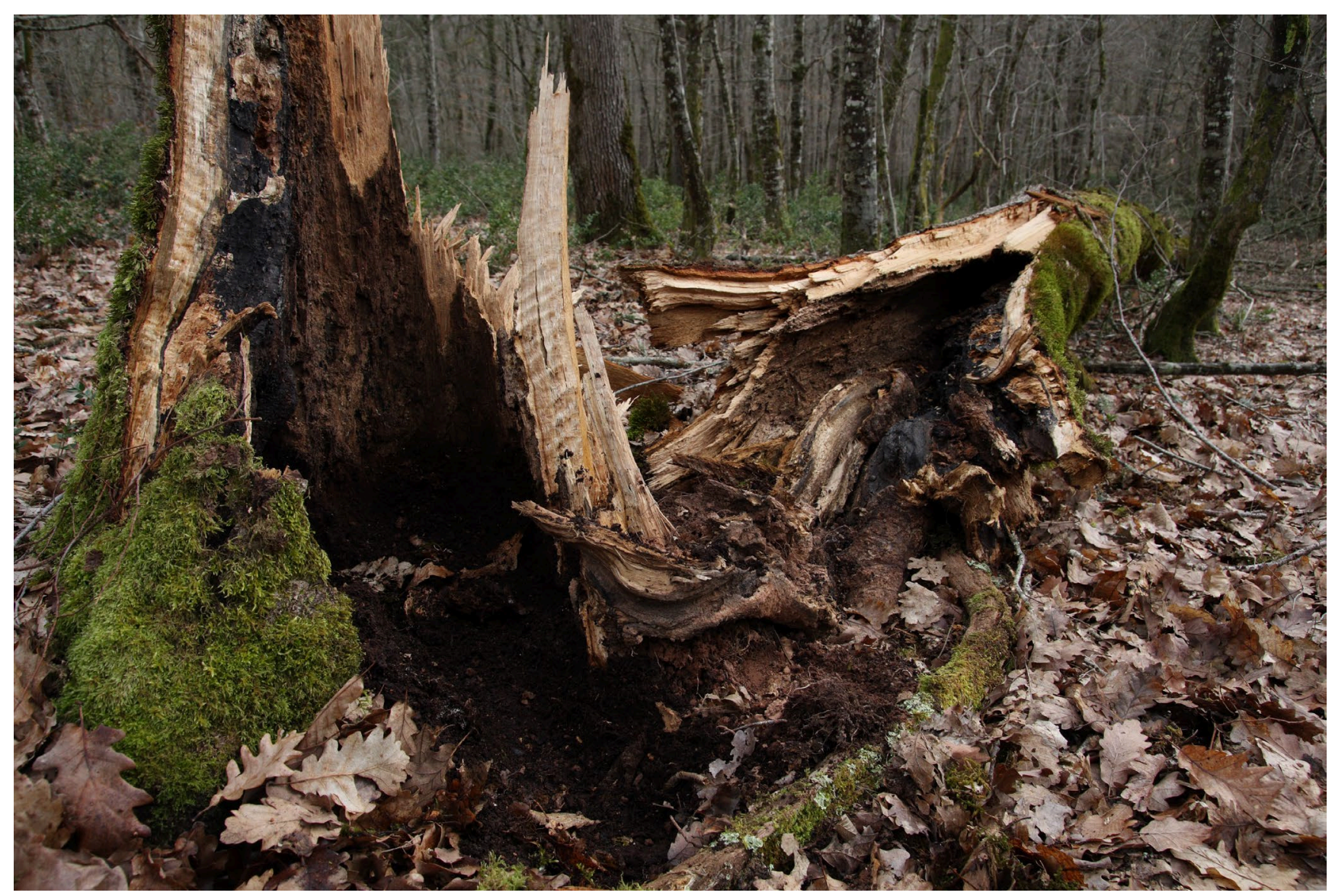

FIG. 6. - Volis d'un arbre habité, fin naturelle d'un habitat à Limoniscus violaceus (P.W.J. Müller, 1821). Crédit photo: N. Gouix.

décennies sur le Pique-prune. Pour atteindre 200 localités en France, ce qui semble plausible à la vue des découvertes par plusieurs entomologistes compétents pour la recherche de Limoniscus violaceus, il faudrait sur 10 ans trouver près de 20 localités par an, soit au moins 30 jours de terrain ciblés sur des secteurs fortement favorables et réalisé par une personne compétente.

Confirmer la présence de l'espèce sur des sites connus est plus simple à organiser. Une vérification tous les 10 ans semble suffisante considérant les vitesses d'évolution des vieux arbres. Cela consiste à vérifier deux sites actuels chaque année pour les dix ans à venir.

La seconde priorité, concerne le suivi de l'habitat d'espèce dans tous les sites où la présence de l'espèce est avérée (Tableau 4). Suivre l'évolution de la disponibilité en cavités basses tous les cinq ans semble pertinent. L'effort nécessaire pour la réalisation des placettes de suivi est d'environ une journée de terrain pour quatre placettes d'un hectare réalisable sur un site localisé (ex: une forêt). Si les sites sont éclatés, 0,5 jours par placette est nécessaire en comptant les trajets.

En résumé, un effort de 40 à 50 jours de terrain par an semble nécessaire pour pouvoir à la fois suivre les sites actuellement connus mais aussi pour tenter de combler les lacunes actuelles sur la distribution de l'espèce en France.
OSMODERMA EREMITA (PIQUE-PRUNE)

\section{Présentation générale}

Le Pique-prune est une espèce cavicole (Fig. 7). L'ensemble de son cycle biologique se déroule dans des cavités à terreau liées à des arbres âgés (Fig. 8).

Il faut beaucoup de temps avant qu'un arbre ne développe une cavité: si des chênes non taillés développent des cavités favorables au Pique-prune entre 150 et 400 ans, il faut entre 70 et 120 ans pour que celui-ci colonise un chêne têtard (Vignon 2006). Forestier, le Pique-prune était une espèce liée aux massifs anciens ayant toujours conservé de vieux arbres. Depuis plusieurs siècles, la gestion des forêts a éliminé les vieux arbres, les arbres blessés et raccourcit l'âge d'exploitabilité. Le Pique-prune a dû sortir de la forêt pour trouver des habitats de substitution et, aujourd'hui, la plupart de ses effectifs se retrouvent en milieu bocager ou en verger, façonnés par l'agriculture traditionnelle (Ranius et al. 2005), surtout en plaine et jusqu'à $1400 \mathrm{~m}$ d'altitude.

L'artificialisation du territoire et les remembrements ont eu pour conséquence la perte et la fragmentation des habitats naturels qui entraine la disparition et l'isolement des populations de Pique-prune. Après la destruction directe de l'habitat, la deuxième cause de disparition de l'espèce est le non renouvellement des arbres à cavités, dont la présence 


\begin{tabular}{llc}
\hline Éléments du protocole & Détection - distribution & Suivi d'habitat - population \\
\hline $\begin{array}{l}\text { 1. Paramètres visés par le protocole et } \\
\text { objectifs détaillés }\end{array}$ & $\begin{array}{c}\text { Nombre de site de présence avérée } \\
\text { de moins de } 10 \text { ans. } \\
\text { Vérifier la présence dans les sites connus et } \\
\text { détecter de nouveaux sites, en cherchant } \\
\text { au moins une cavité occupée par site. }\end{array}$ & $\begin{array}{c}\text { Habitat présent et futur: nombre de cavités } \\
\text { favorables et de futures cavités favorables } \\
\text { dans les sites où l'espèce est connue. }\end{array}$ \\
$\begin{array}{c}\text { Recherche des forêts/bocages avec } \\
\text { des cavités basses favorables } \\
\text { (Gouix et al. 2015): identification des } \\
\text { 2. Plan d'échantillonnage national, }\end{array}$ & $\begin{array}{c}\text { Dans tous les sites connus, dénombrement } \\
\text { choix des sites/placettes }\end{array}$ & $\begin{array}{c}\text { et suivi de la ressource en cavités basses } \\
\text { selon la typologie de Gouix (2011). }\end{array}$ \\
& sitentiels grâce aux inventaires &
\end{tabular}
préexistants (présence d'espèces compagnes).

Vérification du maintien de toutes les stations connues par une recherche ciblée.

3. Unité d'échantillonnage
$\begin{aligned} & \text { Étude de } 20 \text { arbres a priori favorables } \\ & \text { (ou cinq très favorables si moyens limités). }\end{aligned}$

Géoréférencement de tous les arbres à cavités potentiellement favorables (petits sites: échantillonnage en plein); placettes permanentes d'un ha dans les grands sites.

4. Techniques et matériels

a) Examen du terreau sur drap blanc avec précaution sur la remise du terreau dans le cavité (Gouix et al. 2015). Loupe de terrain pour l'identification de la larve.

b) Possibilité de mise en place de pièges à émergence (Gouix 2011).

5. Période(s) favorable(s) $\begin{aligned} & \text { Toute l'année pour la recherche active } \\ & \text { mais préférer la période fin hiver début } \\ & \text { printemps. Avril à mi-juin pour les pièges à } \\ & \text { émergence. }\end{aligned}$

6. Co-variables à relever Diamètre à $30 \mathrm{~cm}$ des arbres et stade d'évolution de la cavité (Gouix 2011) Présence d'autres Taupins et autres coléoptères cavicoles: Cardiophorus, Ischnodes, Megapenthes lugens (W. Redtenbacher, 1842), etc.

7. Technicité/compétence pour effectuer les relevés

Entomologiste confirmé pour la prospection active.

Écologue forestier pour l'identification des secteurs et la relève de pièges à émergence.

8. Possibilité de mobiliser des gestionnaires? Oui pour l'identification des secteurs a priori Suivi à effectuer par les gestionnaires. favorables.

$\begin{array}{ll}\text { 9. Périodicité des relevés } & \begin{array}{c}\text { Tous les } 10 \text { ans (vérification de présence). } \\ \text { Continu pour la recherche de nouvelles } \\ \text { stations. }\end{array} \\ \begin{array}{l}\text { \% de sites où l'espèce est retrouvée. } \\ \text { Évolution du taux d'occupations } \\ \text { des arbres. }\end{array} \\ \begin{array}{l}\text { Une journée par site tous les } 10 \text { ans } \\ \text { (deux journées au minimum avec la } \\ \text { 11. Élémnique d'émergence). }\end{array} \\ \text { 12. Aspect expérimental de coût développer } & \begin{array}{l}\text { Étude des phéromones et/ou kairomones. } \\ \text { Étude de l'ADN environnemental dans le } \\ \text { terreau des cavités. }\end{array}\end{array}$

13. Sites pilotes potentiels
Toute l'année.

visuelle et georéférencement

selon la typologie (Gouix et al. 2015).

Essence, diamètre à $30 \mathrm{~cm}$ et stade d'évolution (Gouix 2011).

Noter la disparition des arbres et la cause (anthropique: coupe, etc., naturel: chablis, volis, etc.).

Compétence en reconnaissance de microhabitats et des essences d'arbres.

Tous les cinq ans.

Densité d'arbres à cavité en formation. Densité d'arbres à cavités favorables.

Courbe d'évolution et tendances.

Une journée de formation du gestionnaire par site, 0,5 jour par site tous les cinq ans (une journée pour les grands sites).

Création d'habitats de substitution. Étude de la distribution spatiale des cavités. était jusqu'alors liée à des pratiques agricoles ancestrales de taille (en têtards ou émonde) aujourd'hui quasi-abandonnées depuis au moins 30 ans. Cette espèce qui disperse peu, en général à quelques centaines de mètres, est très vulnérable à l'éloignement des autres arbres habités ou favorables (Ranius \& Hedin 2001; Hedin 2003; Dubois 2009). Les distances de dispersion mesurées les plus élevées, jusqu'à $1,5 \mathrm{~km}$ (Chiari et al. 2013a), ont été obtenues en milieu méditerranéen et ne sont probablement pas transposables en dehors de cette région, soit sur la majeure partie de l'aire de répartition française de l'espèce qui ne disperse pas ou peu au-delà de $300 \mathrm{~m}$.

Les études sur la capacité de dispersion de l'espèce montrent que seule une faible fraction des adultes (environ 15\% en Suède) d'une même cavité sortent et tentent de se disperser (Ranius \& Hedin 2001). 

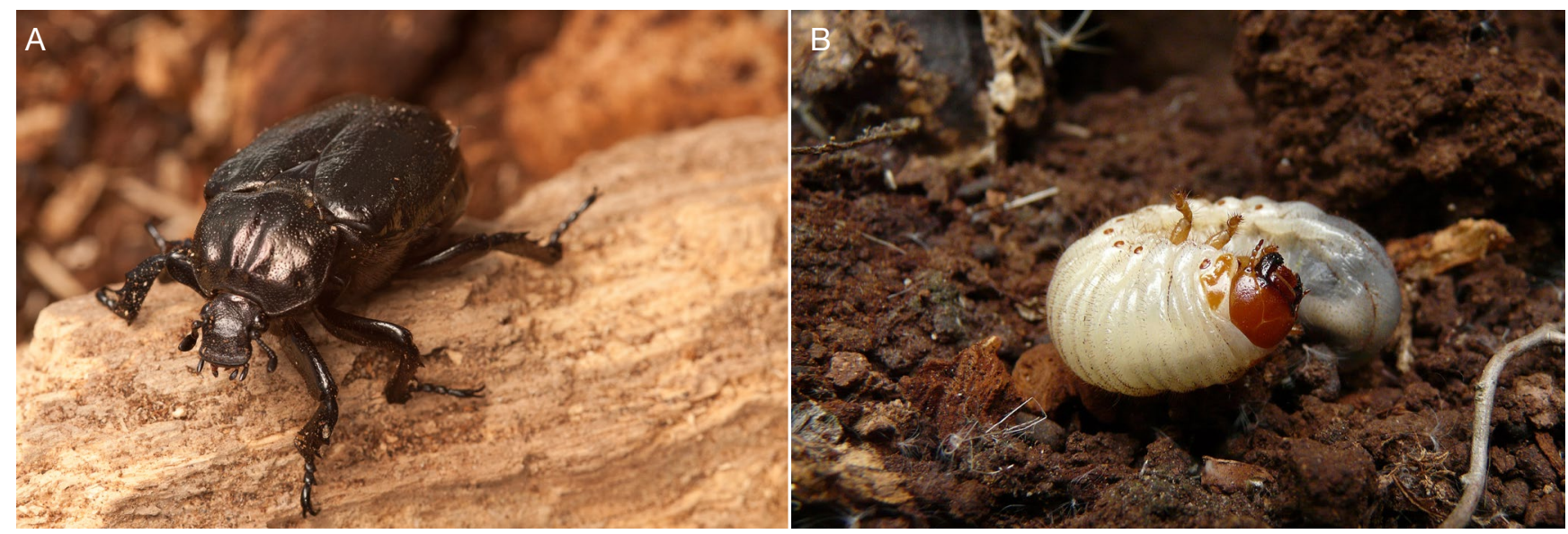

FIG. 7. - Osmoderma eremita (Scopoli, 1763). A, imago; B, larve. Crédits photos: N. Gouix (A) et V. Vignon (B).

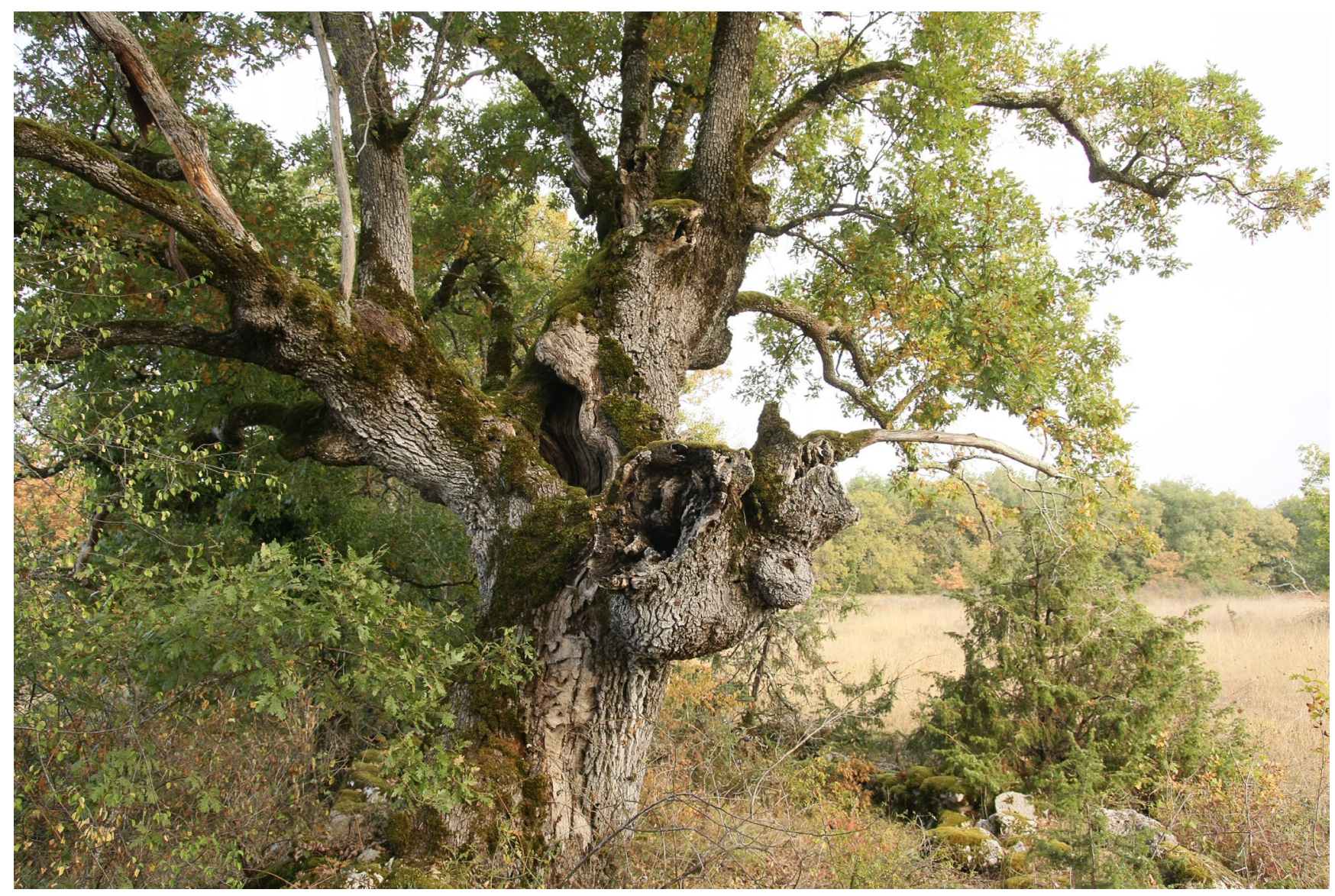

FIG. 8. - Chêne à cavité favorable à une population de Pique-prune, Osmoderma eremita (Scopoli, 1763). Crédit photo: N. Gouix

\section{Paramètres visés par le protocole}

Détection. De nombreux sites sont connus grâce aux inventaires dans le cadre de Natura 2000 et des ZNIEFF, notamment les sites abritant encore de grandes populations. De nouvelles stations, souvent de petite taille (quelques arbres) sont cependant découvertes chaque année.

La détection de nouveaux sites occupés par le Pique-prune peut s'établir en deux phases: un pré-repérage cartographique et/ou un survol aérien de zones a priori favorables qui précède une recherche active sur le terrain d'indices de présence.

L'évaluation des zones jugées favorables se fonde sur la structure du paysage (gros arbres dans le bocage), la présence connue d'espèces cavicoles associées au Pique-prune (comme Elater ferrugineus Linnaeus, 1758, Tenebrio opacus Duftschmid, 1812, etc. ou la Chouette hulotte, Strix aluco Linnaeus, 1758), sur la remontée d'informations citoyennes, etc. 

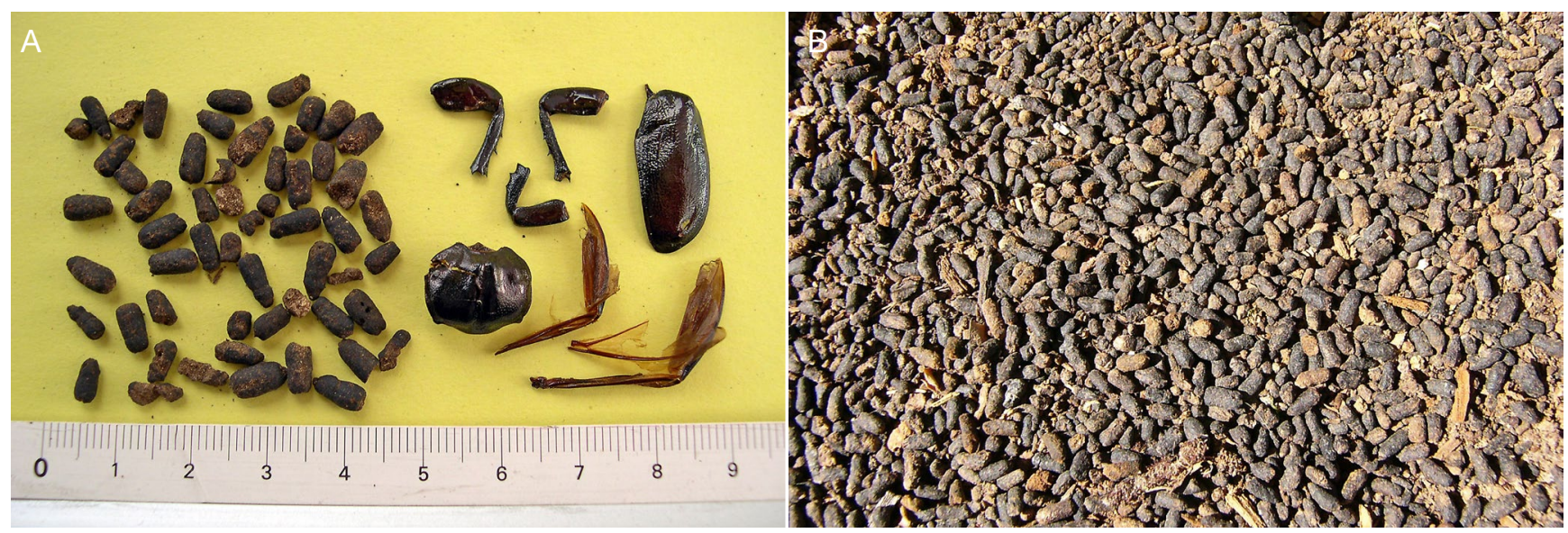

FIG. 9. - A, Fèces des larves et macro-restes d'adultes d'Osmoderma eremita (Scopoli, 1763); B, fèces de larve d'Osmoderma eremita au sol. Crédits photos: V. Vignon.

Le survol aérien doit être effectué lorsque les feuilles sont tombées (en hivers ou en début de printemps pour les forêts à arbres marcescents comme le Chêne pubescent), afin d'avoir une meilleure visibilité (taille des troncs en particulier et forme particulière des arbres taillés en têtard), à une altitude de quelques centaines de mètres (150 à $500 \mathrm{~m}$ ). Il apporte une vision globale du réseau bocager environnant et/ou des secteurs boisés, ainsi que des connexions éventuelles (réseaux de haies). Il permet de s'affranchir du relief, des obstacles à la vue, de l'accessibilité en véhicule, et d'identifier les habitats en vision verticale et oblique jusqu'à $1,5 \mathrm{~km}$ environ (Vignon \& Orabi 2003a). Ce survol permet d'établir un diagnostic et une notation du bocage (Vignon \& Orabi 2002, 2003b) qui permettent ensuite de préparer les prospections de terrain.

Pour une détection rapide, on s'orientera sur les arbres jugés les plus favorables: arbres feuillus de gros diamètre, présentant des cavités avec de grands volumes de terreau (Ranius \& Nilsson 1997).

Nos retours d'expérience suggèrent la nécessité de prospecter une vingtaine d'arbres à cavité favorables pour établir la présence ou l'absence de l'espèce. Le nombre d'arbres à prospecter doit être supérieur dans certains bocages du nord de la France où seulement $1 \%$ des arbres taillés en têtards sont réellement habités par l'espèce (Vignon 2017).

Les observations directes d'individus d'Osmoderma eremita (larves, nymphes ou imagos: Fig. 7) sont difficiles car les larves rongent souvent le bois au plus profond des cavités. Les mâles passent plus de temps en surface que les femelles. Les preuves de présence se résument le plus souvent à la présence des crottes des larves (fèces caractéristiques au dernier stade L3) et de macro-restes d'imagos (pattes, élytres, pronotum, etc.) dans le terreau (Fig. 9). Comme les indices peuvent rester plusieurs années dans une cavité, on peut trouver des indices dans une cavité qui n'est plus occupée par l'espèce.

Les prospections de terrain sont possibles toute l'année. Toutefois des précautions doivent être prises durant les mois d'août et septembre du fait de la présence fréquente de nids de frelons dans les cavités. Des précautions doivent aussi être prises entre octobre et juin lorsque les larves sont en "coque». Celles-ci sont fragiles et peuvent être détruites par une manipulation trop brutale du terreau. L'observation des fèces est possible toute l'année. L'observation des imagos est possible de fin juin à fin août suivant les régions avec un maximum d'activité entre le 10 et le 30 juillet (Vignon 2017). C'est notamment au cours de cette période que les mâles peuvent être détectés à l'odeur au cours des après-midi chaudes.

Si l'entrée de la cavité est située trop haute ou que son accès est trop dangereux, l'utilisation d'une échelle ou de techniques d'escalade des élagueurs voltigeurs (faire appel à un spécialiste formé) sera nécessaire.

Une petite barquette en plastique blanc ou un drap blanc facilitera un tri rapide sur place, des fragments récupérés dans le terreau. Les 10 plus grosses crottes seront gardées pour preuve dans une enveloppe annotée: lieu (point GPS), prospecteur, date. Elles vont se rétracter en séchant.

Suivi des habitats. Pour les sites dont la présence de l'espèce est avérée, un suivi des habitats correspond (équivaut) au suivi de la disponibilité en cavités favorables (habitat de l'espèce) et au suivi du recrutement en cavités (futurs arbres hôtes de l'espèce). Compte tenu du nombre de stations connues (plus de 300), un échantillonnage stratifié par région biogéographiques DHFF et par type de populations est préconisé (Tableau 5). Les stations de suivi seront permanentes.

Compte tenu de la biologie d'Osmoderma eremita, hôte de cavités de feuillus (mais parfois également d'ifs, voire d'autres résineux ailleurs en Europe), c'est à l'échelle de groupes d'arbres favorables que l'étude des populations de cet insecte doit être menée: «L'enjeu majeur consiste aujourd'hui à identifier les sites où les métapopulations sont encore viables et à renouveler les arbres à cavité avant la perte de ceux qui portent les dernières populations" (Vignon 2014). Il conviendra donc de suivre des ensembles d'arbres à cavités entre lesquels des individus peuvent circuler, ceci donnera une indication sur l'état de la métapopulation. De plus les capacités de dispersion de l'espèce semblent limitées: de quelques centaines 


\begin{tabular}{|c|c|}
\hline Éléments du protocole & Détection - distribution \\
\hline $\begin{array}{l}\text { 1. Paramètres visés par le protocole et } \\
\text { objectifs détaillés }\end{array}$ & $\begin{array}{l}\text { Vérification de la présence de l'espèce sur } \\
\text { les sites connus: nombre de sites, taux de } \\
\text { présence, préciser le niveau de certitude } \\
\text { de la présence si la donnée repose sur des } \\
\text { indices de présence. } \\
\text { Recherche ponctuelle de nouveaux sites. }\end{array}$ \\
\hline $\begin{array}{l}\text { 2. Plan d'échantillonnage national, choix des } \\
\text { sites/placettes }\end{array}$ & $\begin{array}{l}\text { Pour la recherche de nouveaux sites: } \\
\text { identification des ZNIEFF avec des } \\
\text { arbres à cavités; présence du cortège de } \\
\text { coléoptères saproxyliques associés ou } \\
\text { d'espèces des arbres matures en milieux } \\
\text { agropastoral (Murin de Bechstein, Myotis } \\
\text { bechsteinii (Kuhl, 1817), Chevêche, Athene } \\
\text { noctua (Scopoli, 1769), etc.). Vérifier les } \\
\text { agrosystèmes en bon état de conservation } \\
\text { où l'espèce était connue même il y a plus } \\
\text { de } 100 \text { ans. }\end{array}$ \\
\hline 3. Unité d'échantillonnage & $\begin{array}{l}20 \text { cavités d'arbres les plus favorables } \\
\text { pouvant être prospectées à pied. }\end{array}$ \\
\hline
\end{tabular}

4. Techniques et matériel

5. Période(s) favorable(s)

6. Variables et co-variables à relever

\section{Suivi d'habitat - population}

Suivi de la ressource en cavités favorables (nombre de cavités, actuelles et futures cavités favorables).

Tirage aléatoire de 30 ZNIEFF ou sites Natura 2000 dans chaque région biogéographique. Stratification en fonction du type de populations: grands sites à nombreux arbres; petits sites; arbres isolés.
Recherche à la main de fèces de larves dans le terreau des arbres à cavité ou sous les cavités. Matériel nécessaire: échelle pliable, GPS-smartphone, enveloppes (prélèvements ou photos d'indices: fèces, débris d'adulte mort).

Période hors feuille (+ survol aérien préalable, To si nécessaire, en hiver également).

Diamètre, essence et stade d'évolution. Point GPS. Photos de l'arbre et des preuves de présence. Faune saproxylique associée (Ranius 2002).
Une station par site: ensemble d'arbres favorables (à cavité ou à future cavité) proches.

Géoréférencement de chaque arbre $(>30 \mathrm{~cm}$ de diamètre) de la station. Observation visuelle des cavités (degré d'avancement de la cavité, volume de terreau estimé). Observation opportuniste de macro-reste (avec photographie et conservation des indices).

Toute l'année avec une préférence pour la période hors feuille.

À l'échelle du site, pour tous les gros arbres (>60 cm de diamètre, ou moins pour les vergers): diamètre, essence et stade d'évolution (pas de cavité/ cavité en formation/cavité favorable): point GPS et photo de l'arbre. De façon occasionnelle, les éléments visibles de la faune associée.

\section{Technicité/compétence pour effectuer les \\ Entomologistes expérimentés pour validation Suivi d'habitat réalisable par le gestionnaire.} relevés des fèces de larves.

Attention à la sécurité: si l'entrée est située trop haute ou que son accès est dangereux avec une échelle, utilisation de technique d'escalade des élagueurs (faire appel à un spécialiste formé).

Oui pour identification des secteurs Oui, après formation préalable.

8. Possibilité de mobiliser des opérateurs gestionnaires?

favorables et repérage des arbres à cavités.

Avis d'un expert pour la détection de l'espèce.

9. Périodicité des relevés $\quad$ Tous les 10 ans. Dén

10. Type d'indicateur et d'analyse

\% de sites où l'espèce est retrouvée.

Évolution du taux d'occupation des arbres (sur 20 arbres).

11. Éléments de coût

Une journée par site tous les 10 ans (à deux personnes si grimpeur + entomologiste). De 10 arbres en milieux escarpés ou embrousaillés à 20 arbres/jours en bocage de plaine.

12. Aspect expérimental à développer

Test des pièges à phéromones (Valainis et al. 2015). Possibilité de détection par ADN environnemental. Descripteur (indicateurs indirects) d'occupation de la cavité.

13. Sites pilotes potentiels

Test dans les sites les mieux répertoriés, où les arbres abritant des populations sont géoréférencés: site Natura 2000 FR7302001, PNR des Boucles de la Seine normande, Parc national des Cévennes, Réserve naturelle de la Massane, Site Natura 2000 FR5202005 des Châtaigneraies de la Sarthe. 


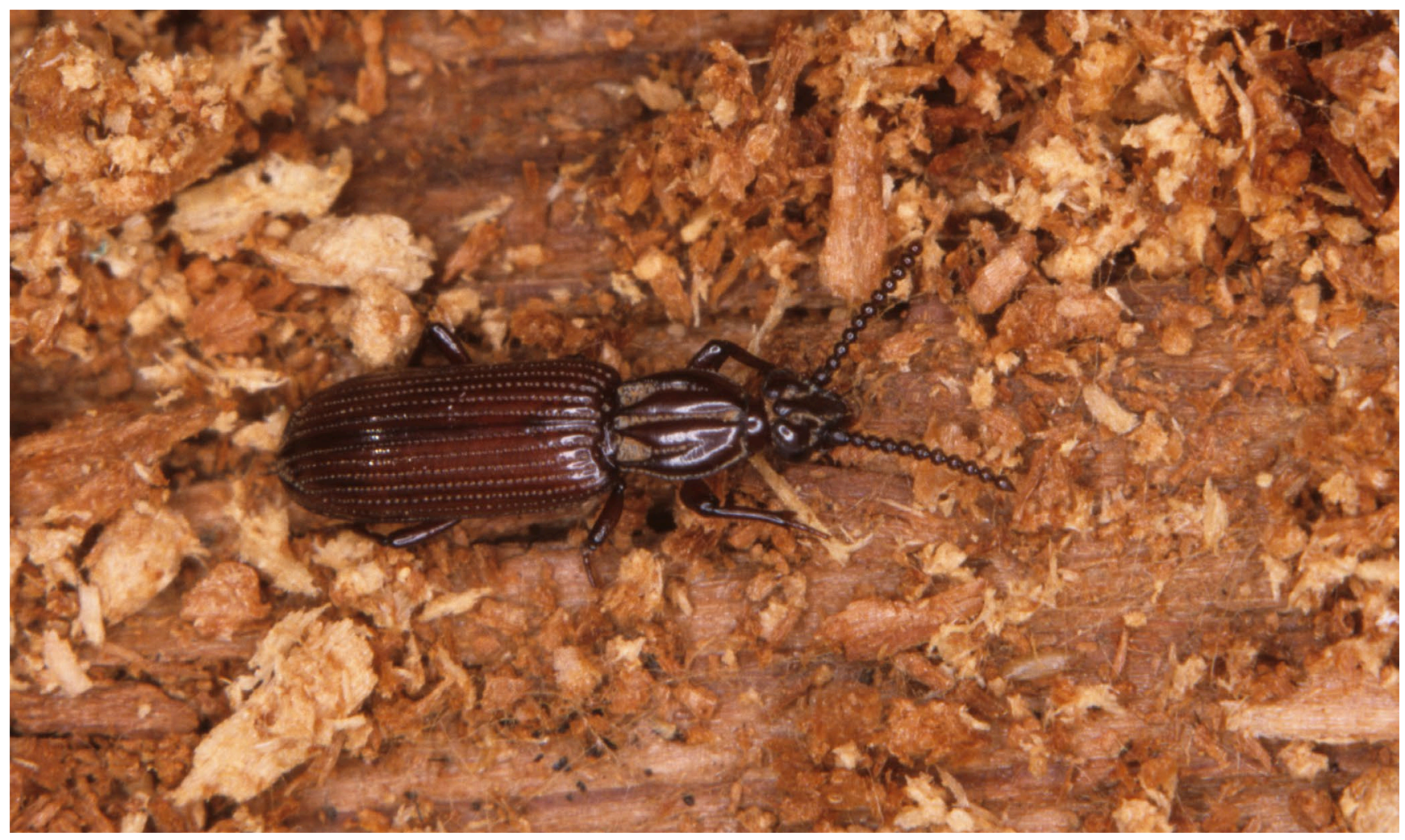

FIG. 10. - Imago de Rhysodes sulcatus (Fabricius, 1787). Crédit photo: H. Bouyon.

de mètres observées en Suède et dans le nord de la France (Ranius \& Hedin 2001; Hedin 2003; Dubois 2009) à 1,5 km en conditions méditerranéennes en Italie (Chiari et al. 2013a). Ces points de connaissance mettent en avant l'importance de chacun des arbres occupés par l'espèce. La notion de station, unité correspondant à une population probable et à un site de suivi, peut être assimilée à un polygone reliant l'ensemble des gros arbres (en général $>60 \mathrm{~cm}$ de diamètre et feuillus, règle à adapter dans les cas de milieux particuliers peu productifs ou pour les pommiers en verger qui peuvent abriter l'espèce dès $30 \mathrm{~cm}$ de diamètre), à cavité formée ou pas, distants les uns des autres de moins de $500 \mathrm{~m}$. En cas de discontinuité de plus de $500 \mathrm{~m}$, il faut considérer deux stations.

Compte tenu de la vitesse de disparition des arbres à cavités dans le bocage, un suivi tous les cinq ans est préconisé (Tableau 5).

\section{Orientations pour la mise en place}

d'une surveillance nationale

De nombreuses stations sont connues et c'est aujourd'hui leur pérennité à court et long terme qui est l'enjeu majeur. C'est ainsi le suivi de l'habitat qui constitue la priorité, par rapport à la détection de nouvelles stations. Les nouvelles stations pourront cependant être recherchées dans le cadre du programme de connaissance ZNIEFF et pourront bénéficier des observations fournies par les entomologistes, y compris de façon marginale par des observations du grand public.

Au-delà d'une approche de surveillance par échantillonnage, le suivi des arbres habitats pourra être mené par les gestionnaires dans l'ensemble des sites de l'espèce.

\section{RHYSODES SULCATUS}

Rhysodes sulcatus (Fig. 10) est une espèce d'Europe du Sud et centrale signalée depuis l'Espagne jusqu'au Caucase et l'Ouest Sibérien. Montagnarde en Europe du Sud, l'espèce est présente en plaine au nord de son aire de répartition. En France, les données récentes établissent que Rhysodes sulcatus prospère dans trois noyaux de population distincts: dans le Massif central dans les gorges de la Rhue (Cantal) et dans les Pyrénées, d'une part autour de la haute vallée de l'Aude (Aude et Pyrénées-Orientales) et d'autre part dans les Pyrénées occidentales depuis la vallée d'Ossau jusqu'aux Aldudes (Pyrénées-Atlantiques) (Brustel \& Gouix 2011; https:// inpn.mnhn.fr/viewer-carto/especes/222951).

Des mentions anciennes au Mont-Pilat (Loire), à Auroux (Lozère) et en forêt de Rhonnes ou Ronne (Savoie) doivent inciter à rechercher cette espèce cryptique dans l'est du Massif central et les Alpes. Ceci est un enjeu de taille car de nouvelles populations peuvent être découvertes comme ce fut le cas récemment dans les gorges de la Rhue.

Dans la majorité des cas, ce sont les grosses pièces cariées de bois de Sapin pectiné (Abies alba Mill.) au sol (mais autres essences possibles) qui intéressent le Rhysodes sulcatus, principalement dans les hêtraies-sapinières (Fig. 11). Dans les secteurs où l'espèce est connue, son observation est à la portée de tous les entomologistes expérimentés s'il y a suffisamment de grumes cariées. Ainsi, il peut être fréquent et abondant dans les chablis réguliers des couloirs d'éboulis de la vallée d'Ossau, sur les grumes abandonnées en forêt d'Iraty, ou dans les cimetières de Sapin pectiné en limite écologique trop sèche et basse des forêts de l'Aude. 


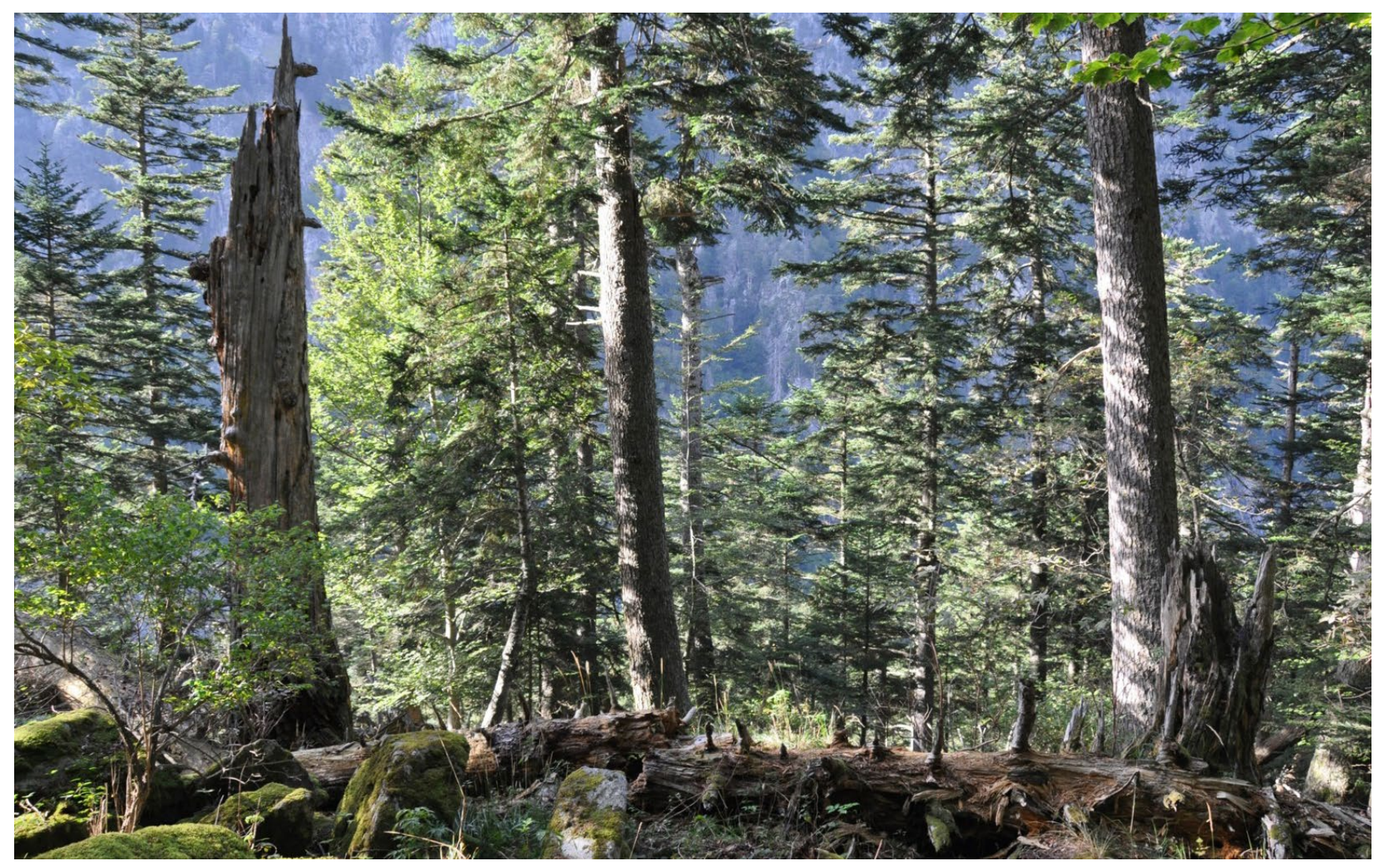

FIG. 11. - Tronc de Sapin pectiné (Abies alba Mill.) au sol, micro-habitat typique de Rhysodes sulcatus (Fabricius, 1787) pouvant être le support d'une autre espèce DHFF: la Buxbaumie verte (Buxbaumia viridis (Moug. ex Lam. \& DC.) Brid. ex Moug. \& Nestl.). Crédit photo: J.-M. Savoie.

\section{Paramètres visés par le protocole}

Détection. Il convient de rechercher cette espèce en zones collinéennes et montagnardes fraiches dans les derniers lambeaux de "vieilles forêts» françaises (= subnaturelles = anciennes et très matures, Cateau et al. 2015) occupés par des essences de dryades (Sapin pectiné, Hêtre Fagus sylvatica L., Chêne sessile Quercus petraea Liebl.) et présentant un stock de bois mort au sol conséquent ( $>80 \mathrm{~m}^{3} \mathrm{ha}$, a priori) : dans ces rares situations la recherche de nouveaux sites à Rhysodes sulcatus présente de réelles chances de succès et mérite d'être tentée. Idéalement il s'agira de forêts non exploitées de plusieurs dizaines d'hectares, sinon des massifs exploités mais alors très raisonnablement, très boisés et de plusieurs centaines d'hectares.

Le Rhysodes sulcatus est visible a priori toute l'année, bien qu'il semble plus profondément enfoncé dans son substrat en hiver, à l'état imaginal et par petites colonies d'individus grégaires le plus souvent. Les grosses grumes au sol de plus de $60 \mathrm{~cm}$ de diamètres humides et très cariées (stade 4 idéalement: Fig. 11) sont à rechercher (Kostanjsek et al. 2018) : l'essence et la nature de la carie importent peu mais d'expérience, ce sont les grosses grumes de Sapin pectiné dont la carie rouge cubique humide a entièrement colonisé qui concentrent la majorité des observations connues en France. Parfois l'espèce peut être sur Hêtre ou d'autres essences, en situation sous-corticale, exceptionnellement sur chandelle et aussi sur grumes de bois morts au sol de plus petites sections (dès $25 \mathrm{~cm}$ ) quand les grosses grumes semblent ponctuellement plus rares (H. B. \& L. V. obs. pers en France). Les larves sont rarement observées bien que le cycle soit de deux ans (Burakowski 1975).

L'espèce présente toutes les caractéristiques d'un taxon à faible pouvoir de dispersion. Les adultes sont nocturnes et déambulent sur leur substrat et ils peuvent voler (Kostanjsek et al. 2018). Comme Rhysodes semble être un piètre foreur, il privilégie le cheminement sous-cortical ou entre les fissures des bois cariés, en particulier celles caractéristiques des caries rouges cubiques. Le piège vitre ou la prospection à la lampe sont possibles mais moins efficaces que de sonder du bois carié au piochon. Cette prospection intrusive est à limiter aux quelques meilleures grumes potentielles d'un site et en se limitant à 1 à $15 \mathrm{mn}$ maximum sur un seul gros tronc en fonction du potentiel et du succès du sondage (Kostanjsek et al. 2018; Fig. 12). Elle doit également se limiter à la recherche sur le tiers du volume de carie. Les recherches "à la belle saison" (mai à début novembre) sont à privilégier car les imagos sont en général moins profondément cachés dans le bois carié et la recherche peut s'avérer moins intrusive. En dehors des caractéristiques a priori favorables de la grume, la présence de certains taxons associés peut orienter les recherches: les Trogossitidae Peltis ferruginea (Linnaeus, 1758), Peltis grossa (Linnaeus, 1758) et Grynocharis oblonga (Linnaeus, 1758); les Elateridae Ampedus melanurus Mulsant \& Guillebeau, 1855, Ampedus elegantulus (Schönherr, 1817) ou Lacon lepidopterus (Panzer, 1800); Prostomis mandibularis (Fabricius, 1801) (Prostomidae); des larves de Xanthochroa spp. (Oedemeridae). 
A

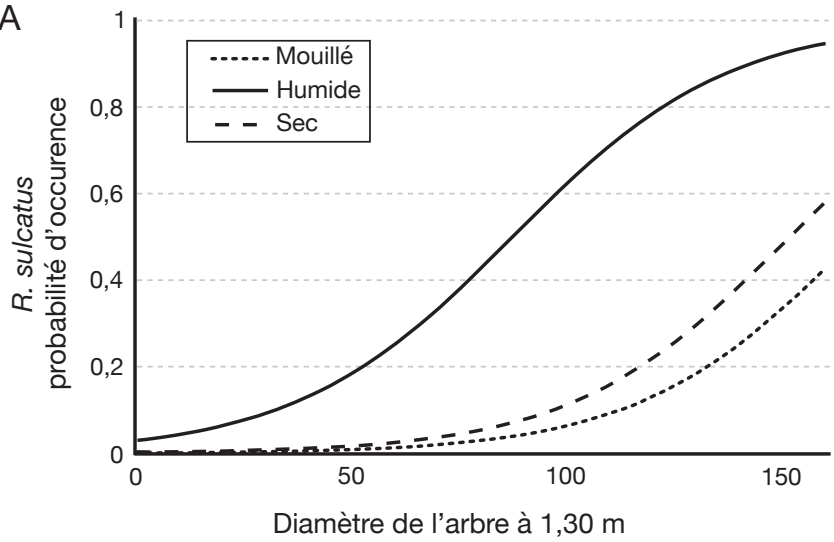

$\mathrm{B}$

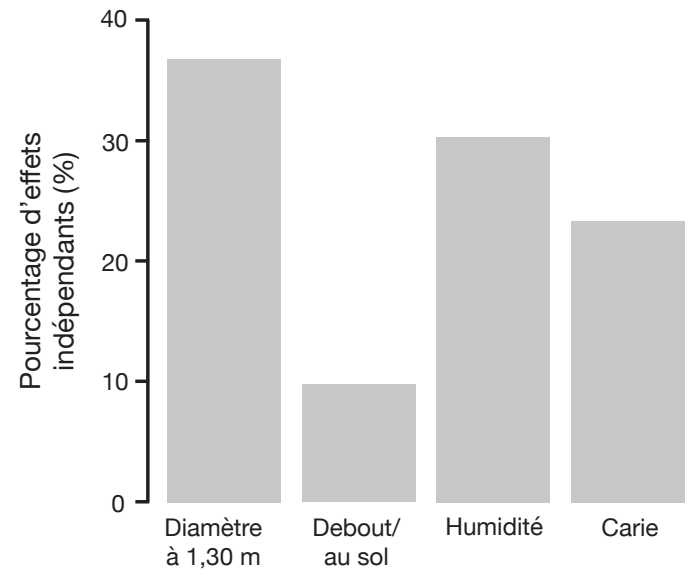

FIG. 12. - A, Probabilité d'occurrence de Rhysodes sulcatus (Fabricius, 1787) dans une pièce de bois mort en fonction de son diamètre et de son humidité; B, contribution indépendante de chaque indicateur à sa probabilité de présence: diamètre, carie, debout/au sol, humidité (repris de Kostanjsek et al. 2018, avec l'autorisation des auteurs).

Suivi des habitats et des populations. Nous ne disposons pas d'assez de connaissances pour parler de dynamiques de populations mais il est clair que les dernières populations viables de l'espèce sont partout en Europe liées à des espaces où le taux de boisement et la part de forêts semi-naturelles en libre évolution sont conséquents. Le plus souvent il s'agit de parcs et de réserves intégrales, sauf en France où l'intensification de la récolte dans les massifs où il est connu place peut-être déjà cette espèce en dette d'extinction (gorges de la Rhue, forêt de Gesse, vallées d'Ossau et d'Aspe, etc.). Dans tous les cas, c'est la continuité des grosses grumes au bon stade qui compte et qu'il faut mesurer dans un protocole de suivi. D’expérience et sans mesure précise, la durée d'intérêt optimal des grosses grumes de sapin d'environ $60 \mathrm{~cm}$ de diamètre est d'au moins 15 ans, peut-être le double si l'on intègre une phase non optimale. Il est donc important que la structure (inéquienne) et la dynamique du peuplement (maturation, succession) permette de générer en continue et suffisamment de gros arbres morts. En forêts en libre évolution, les zones de recrutement réguliers de bois morts (zones soumises aux accidents climatiques: chablis, foudre, couloir d'avalanche), parfois peu boisées sont favorable par l'effet du réchauffement solaire de la concentration et de la continuité des grumes favorables.

Dans un site donné, un cheminement d'au moins une demijournée permet à un entomologiste confirmé de diagnostiquer le potentiel de présence de Rhysodes. La preuve d'occurrence peut demander une journée à deux entomologistes confirmés en fonction de la qualité des grumes, de l'abondance temporelle du Rhysodes et de l'accessibilité (pente, pistes, etc.). Un inventaire des vieux peuplements, des points de mesure d'IBP (Indices de Biodiversité potentielle, Larrieu \& Gonin 2008) et les repérages des secteurs de vieux chablis ou volis de très gros bois par les gestionnaires optimisent par la suite les prospections des spécialistes. L'évolution de la qualité d'accueil d'un boisement (parcelle/forêt/massif) est donc synonyme de quantités de grosses grumes cariées dans le temps. Pour s'en rendre compte, un cheminement défini par le tracé des prospections initiales ou par transects assez longs (au moins $2 \mathrm{~km}$ cumulés, si la topographie le permet) peut être réitéré tous les cinq ans avec caractérisation et dénombrement des pièces de bois favorables (stades 3-4) et à terme favorables (bois morts frais et chandelles). Les temps mis par un observateur expérimenté à trouver chacune des trois premières colonies d'un secteur pourrait permettre d'établir un indice de densité.

Orientation pour la mise en place d'une surveillance nationale L'espèce étant rare et localisée, nous préconisons avant tout un suivi "en plein" de l'ensemble des massifs connus, avec un pas de temps de 10 ans (Tableau 6), pour :

- vérifier le maintien de l'espèce;

- localiser les points de présence et cartographier les secteurs favorables;

- quantifier un proxy de l'abondance, à travers le taux de détection par heure de recherche.

L'approche recommandée serait couplée à un transect de longueur standardisée avec relevé des bois morts au sol et des chandelles (Tableau 6).

Les prospections ciblées de nouvelles stations doivent être réservées à deux cas: des stations proches des localités connues et des sites répondant aux caractéristiques d'habitat décrites précédemment.

\section{STEPHANOPACHYS SPP.}

Trois espèces de ce genre sont présentes en France, toutes liées aux résineux. Une d'entre-elles, Stephanopachys quadricollis (Marseul, 1878), plus commune que les deux autres, distribuée dans le sud de l'Europe et le pourtour méditerranéen, ne bénéficie pas d'une inscription en annexe de la DHFF. Cette dernière existe jusque dans des secteurs plus frais des Alpes et se retrouve dans l'aire de distribution des deux autres espèces présentées ci-après. Le recours à un spécialiste pour distinguer ces espèces est souhaitable bien que la détermination soit à la portée de tout naturaliste disposant d'une loupe binoculaire et des clés de détermination (Brustel et al. 2013).

Stephanopachys linearis (Kugelann, 1792) (synonyme de S. elongatus Paykull, 1800) et Stephanopachys substriatus (Paykull, 1800) sont deux espèces montagnardes qui évoluent dans différents résineux (Lesne 1904, 1909; Leseigneur 1951; 
TABLEAU 6. - Synthèse des protocoles recommandés pour une surveillance nationale de Rhysodes sulcatus (Fabricius, 1787).

\begin{tabular}{|c|c|c|}
\hline Éléments du protocole & Détection - distribution - population & Suivi d'habitat \\
\hline $\begin{array}{l}\text { 1. Paramètres visés par le protocole et } \\
\text { objectifs détaillés }\end{array}$ & $\begin{array}{l}\text { Nombre de sites de présence avérée } \\
\text { (confirmation et nouveaux sites). } \\
\text { Taux d'occupation dans les habitats } \\
\text { favorables. }\end{array}$ & $\begin{array}{l}\text { Quantité d'habitat de qualité: nombre (et } \\
\text { volume) de grosses grumes cariées au sol, } \\
\text { dans un rayon proche }(<1 \mathrm{~km}) \text { des points } \\
\text { de présence avérés. }\end{array}$ \\
\hline $\begin{array}{l}\text { 2. Plan d'échantillonnage national, } \\
\text { choix des sites/placettes }\end{array}$ & $\begin{array}{l}\text { Tous les sites potentiels. } \\
\text { Tous les massifs contenant ou proches des } \\
\text { sites avérés (rayon } 20 \mathrm{~km} \text {, parcelles à IBP > } \\
40 \text {, inventaires vieilles forêts). } \\
\text { Prospection de nouveaux sites selon logique } \\
\text { opportuniste. }\end{array}$ & $\begin{array}{l}\text { Tous les massifs où l'espèce est présente. } \\
\text { Une station dans chaque parcelle forestière } \\
\text { de présence avérée. Un transect de } \\
\text { mesures dendrométriques par station. }\end{array}$ \\
\hline 3. Unité d'échantillonnage & $\begin{array}{l}\text { Massif, recherche sur } 20 \text { grumes au sol } \\
\text { favorables. }\end{array}$ & $\begin{array}{l}\text { Transect géoréférencé (permanent): } \\
\text { cheminement adapté à la pente et aux } \\
\text { accidents topographique) de } 1 \mathrm{~km} \text { avec } \\
\text { relevé de l'ensemble des grumes et } \\
\text { chandelles dans le champ de vision. }\end{array}$ \\
\hline 4. Techniques et matériel & $\begin{array}{l}\text { Recherche au piochon sur toute l'épaisseur } \\
\text { de la carie (15' max par grume; arrêt dès } \\
\text { découverte d'un individu). }\end{array}$ & $\begin{array}{l}\text { Observation visuelle et avec un compas } \\
\text { forestier. }\end{array}$ \\
\hline 5. Période(s) favorable(s) & $\begin{array}{l}\text { Toute l'année (conseil de mai à novembre, } \\
\text { hors grumes gelées ou neige). }\end{array}$ & Toute l'année hors neige. \\
\hline 6. Variables et co-variables à relever & $\begin{array}{l}\text { Présence/non détection du Rhysodes. } \\
\text { Diamètre, essence et ouverture du milieu. } \\
\text { Point GPS. Espèces saproxyliques } \\
\text { associées. } \\
\text { Temps passé, temps pour découvrir chaque } \\
\text { colonie. }\end{array}$ & $\begin{array}{l}\text { GB, TGB, TTGB morts: essence, diamètre, au } \\
\text { sol /debout, stade de dégradation, degré } \\
\text { d'ouverture (troué vs fermé) autour de la } \\
\text { pièce de bois. }\end{array}$ \\
\hline $\begin{array}{l}\text { 7. Technicité/compétence } \\
\text { pour effectuer les relevés }\end{array}$ & $\begin{array}{l}\text { Entomologiste confirmé. Écologue forestier } \\
\text { pour l'identification des secteurs à } \\
\text { prospecter. }\end{array}$ & Compétences forestières. \\
\hline $\begin{array}{l}\text { 8. Possibilité de mobiliser } \\
\text { des opérateurs gestionnaires? }\end{array}$ & $\begin{array}{l}\text { Oui pour l'identification des secteurs } \\
\text { favorables. }\end{array}$ & $\begin{array}{l}\text { Oui: forestier et gestionnaires d'espaces } \\
\text { naturels. }\end{array}$ \\
\hline 9. Périodicité des relevés & $\begin{array}{l}\text { Confirmation tous les } 12 \text { ans des sites avérés } \\
+ \text { programme glissant, annuel permettant } \\
\text { de tester des nouvelles stations. }\end{array}$ & Tous les 10 ans. \\
\hline 10. Type d'indicateur et d'analyse & $\begin{array}{l}\text { Découverte ou pas. } \\
\text { Taux d'arbres occupés (/20 arbres). } \\
\text { Temps pour trouver la première colonie et les } \\
\text { trois premières. }\end{array}$ & $\begin{array}{l}\text { Abondance et volume/transect (grumes au sol } \\
\text { et chandelles par types de diamètre, caries, } \\
\text { debout/au sol, etc.). }\end{array}$ \\
\hline 11. Éléments de coût & $\begin{array}{l}\text { Une journée par massif à deux } \\
\text { entomologistes confirmés. GPS ou } \\
\text { smartphone. }\end{array}$ & $\begin{array}{l}\text { Temps de terrain (à coupler avec révision } \\
\text { d'aménagement ou de plan de gestion, ou } \\
\text { avec la phase de détection) + exploitation } \\
\text { des résultats GPS. } \\
\text { Si des données dendrométriques préexistent } \\
\text { = temps d'analyse. } \\
\text { Compter deux jours par massif tous les } 10 \text { ans. }\end{array}$ \\
\hline 12. Aspect expérimental à développer & $\begin{array}{l}\text { Recherches génétiques de taxons } \\
\text { co-occurents (bactéries, champignons, } \\
\text { etc.) avec Rhysodes sulcatus sur substrats } \\
\text { habités ou pas. }\end{array}$ & $\begin{array}{l}\text { Tester les relations entre IBP, surfaces } \\
\text { terrières (bois debout/bois au sol), grumes } \\
\text { potentielles, cheminement optimum. }\end{array}$ \\
\hline 13. Sites pilotes potentiels & Vallée d'Ossau et vallée de Gesse. & \\
\hline
\end{tabular}

Simon 1952; Brustel et al. 2013). Ces deux espèces sont inscrites à l'annexe II de la DHFF depuis 2004 et seront traitées ici ensemble en raison de leurs distributions et biologies relativement semblables. Ce sont deux espèces encore très méconnues en France. Cette méconnaissance s'explique en grande partie par leur caractère cryptique, parce que l'intérêt suscité par leur statut est relativement récent et surtout parce qu'il s'agit d'espèces peu spectaculaires (Fig. 13).

Ces deux espèces boréoalpines sont largement répandues dans le nord de l'aire paléarctique et sont représentées en France par des populations en limite sud d'aire de répartition. Stephanopachys linearis est connue de moins de dix communes françaises: dans les Hautes-Alpes (cinq communes dans le massif du Queyras et Freissinières en haute Durance: Braud et al. 2017), les Pyrénées-Orientales (Vernet-les Bains) et la Corse (Brustel et al. 2013). Stephanopachys substriatus, plus commune, est connue en France seulement dans deux départements alpins, les Alpes-de-Haute-Provence et les Hautes-Alpes, depuis Villard-Saint-Pancrace (Briançonnais) au nord jusqu'à Colmars-les-Alpes (Haut-Verdon) au sud, et de Chorges (Gapençais) à l'ouest jusque dans le Queyras et La Condamine-Chatelard (Ubaye) à l'est (Braud et al. 2017). Les données connues sont visibles et mises à jour en ligne dans l'INPN. 
A

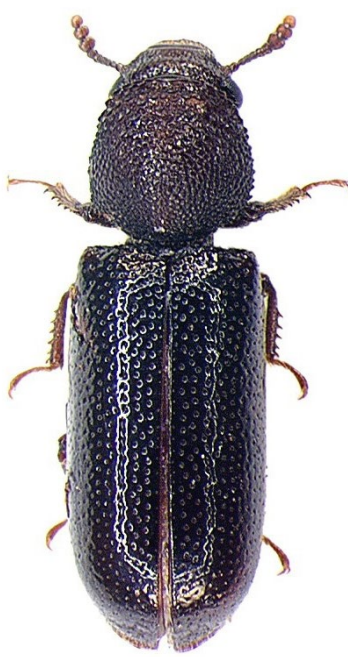

B

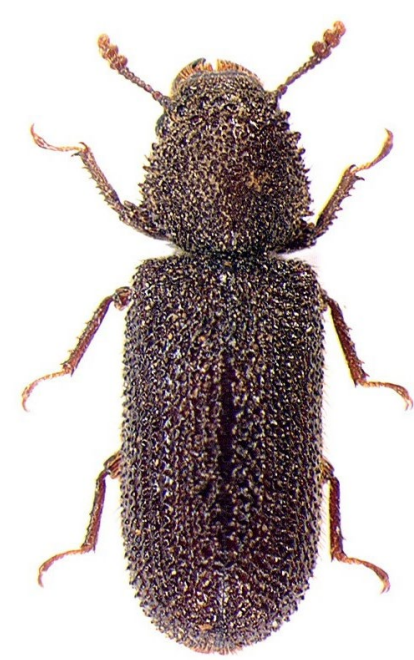

FIG. 13. - A, Habitus de Stephanopachys linearis (Kugelann, 1792) (4,8 mm); B, habitus de Stephanopachys substriatus (Paykull, 1800$)$ (5 mm). Crédits photos: P. Zagatti.

De mœurs saproxyliques, les Stephanopachys spp. sont inféodés aux assises cambiales mortes des écorces déhiscentes de résineux, préférentiellement le Mélèze (Larix decidua Mill.). Les dendro-microhabitats favorables sont rares et éphémères, ce qui rend la détection d'autant plus difficile. Les récents efforts de prospections focalisés sur ce genre dans les Alpes (2015-2017) ont permis de découvrir de nouvelles stations pour Stephanopachys substriatus, dans son habitat sous-corticole ou en activité imaginale (vol, marche) sur ou à proximité de tas de grumes exploitées. Stephanopachys linearis est encore plus exceptionnellement observé ce qui tend à confirmer son statut d'espèce plus rare ou davantage sténoèce, avec un caractère pyrophile prononcé (Brustel et al. 2013; Müller et al. 2013; Braud et al. 2017).

\section{Paramètres visés par le protocole}

L'enjeu prioritaire est de les connaitre mieux et de les faire connaitre. Il s'agit de préciser la distribution par la découverte de nouvelles stations et de préciser l'autécologie, par l'étude de la réponse de ces deux espèces à l'offre de dendro-microhabitats créés artificiellement. Les connaissances actuelles, aires de distribution et exigences écologiques connues, ne permettent pas réellement de suivi des populations. Au mieux, les peuplements a priori favorables peuvent donner lieu à des tentatives de délimitation, de qualification et de quantification (surfaces).

Détection. Les Stephanopachys sont très strictement inféodés aux coniferes, plutôt sur de gros bois qui offrent une épaisseur d'écorce suffisante.

- Stephanopachys linearis sur Pin sylvestre (Pinus sylvestris L.), Épicéa (Picea abies (L.) H.Karst.), Sapin pectiné, Mélèze et probablement aussi sur Pin noir laricio (Pinus nigra subsp. laricio Maire) (en Corse);

- Stephanopachys substriatus sur Pin sylvestre, Pin cembro (Pinus cembra L.), Épicéa et surtout Mélèze.
En Scandinavie, Stephanopachys substriatus est avant tout lié à l'Épicéa alors que Stephanopachys linearis préfère le Pin sylvestre, dans la très grande majorité des observations sur bois brûlés, bois pouvant être attractifs plusieurs années consécutives (Ehnström \& Axelsson 2002). En France, ces deux Stephanopachys préferent clairement les Mélèzes, dans les écorces épaisses sur arbres coupés et endommagés accidentellement. Dans tous les cas, seules les chandelles sont attractives (les grumes au sol le sont seulement très peu de temps). Ces espèces ont les traits de taxons à bonne capacité de dispersion.

Deux types de conditions d'observation représentent la quasi-totalité des données acquises sur ces deux espèces en France et à l'étranger (les pièges vitres semblent inopérants sinon trop aléatoires):

- la découverte de petites populations à l'état larvaire, imaginal ou à différents stades dans l'épaisseur cambiale et corticale de résineux morts debout (chandelles) ou blessés, brulés ou pas, à n’importe quelles périodes de l'année;

- l'observation d'adultes actifs, volant ou marchant, sur ou à proximité d'arbres fraichement coupés (le plus souvent des tas de bois exploités parfois distants des peuplements sources) de juin à août.

Les trois espèces françaises montrent des signatures communes dans l'exploitation de leur substrat de développement (Fig. 14). Elles se développent toutes dans le cambium sous l'écorce de bois morts, ou déficients et encore vivants: sous écorces déhiscentes, dans ou proche de blessures occasionnées par les travaux d'exploitation forestière, le dévalement de blocs rocheux, les chablis ou la foudre, etc. Les attaques sont très caractéristiques et ne sont visibles que sur la face interne de l'écorce. Parfois, quelques exsudats extérieurs de sciures caractéristiques trahissent l'activité corticale de ces espèces.

Ces traces laissées par l'activité cambiophage de ces deux espèces (galeries, trous d'émergence, Fig. 14) peut éventuellement inspirer quelques spécialistes. Cependant, il est 


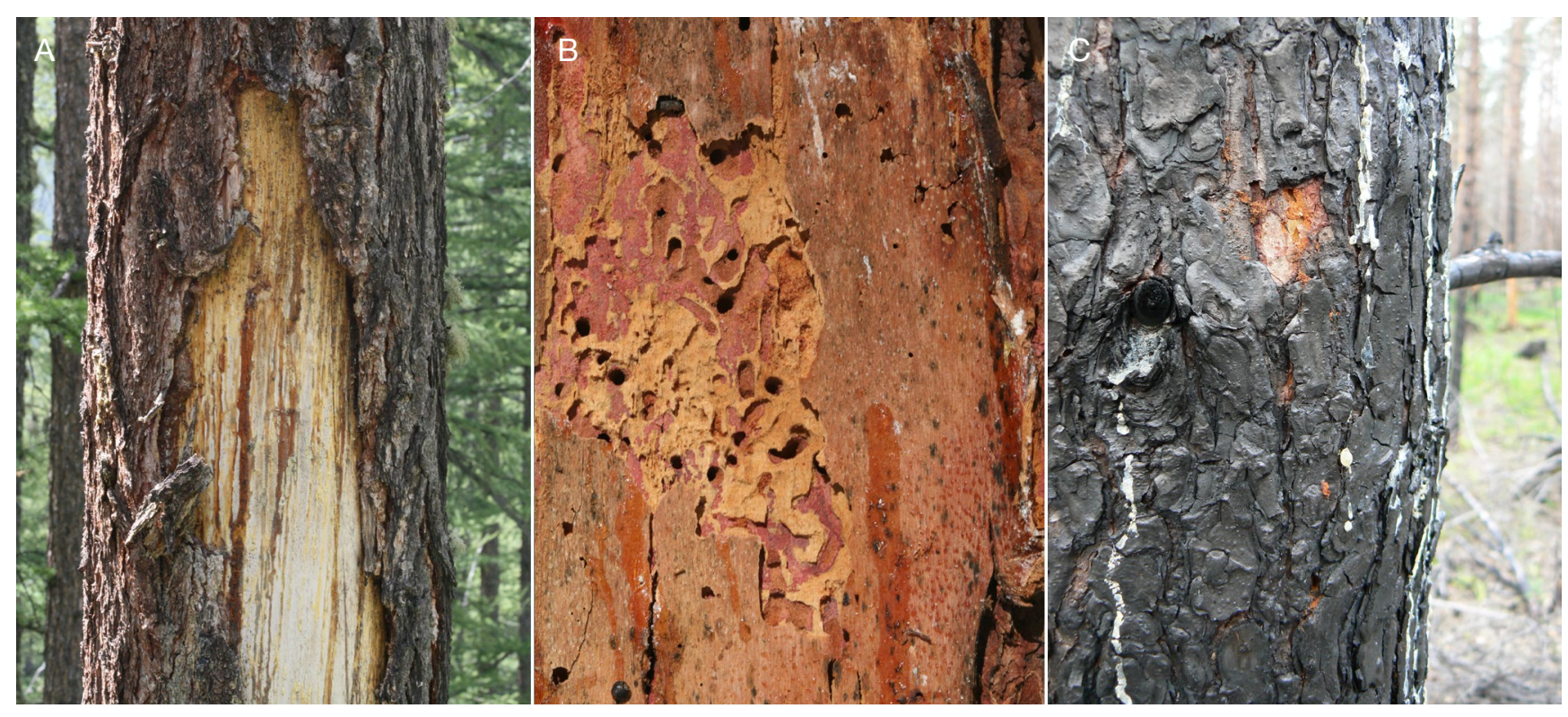

FIG. 14. - Traces et micro-habitats de Stephanopachys linearis (Kugelann, 1792) et de Stephanopachys substriatus (Paykull, 1800): A, habitat sur Mélèze (Larix decidua Mill.) dans le Queyras; B, traces de S. substriatus sous écorce de Mélèze dans le Queyras; C, traces de S. linearis sur Pin sylvestre (Pinus sylvestris L.) brûlé en Finlande. Crédits photos: H. Bouyon (A et B) et $\mathrm{H}$. Brustel (C).

impossible de fonder un diagnostic fiable sans observer des macro-restes d'adultes ou des individus vivants, car d'autres xylophages (Ernobius spp., Nothorhina muricata (Dalman, 1817), Scolytes, etc.) peuvent occuper des niches écologiques semblables et laisser des traces comparables. Les larves des Stephanopachys spp. présentent des pattes bien visibles à la différence des larves des Scolytes qui peuvent coloniser les mêmes arbres (Ehnström \& Axelsson 2002).

Perspectives de suivi des habitats et des populations. Les Stephanopachys semblent être caractéristiques des vieilles futaies de conifères, en particulier les mélézins, avec des arbres qui présentent une épaisseur d'écorce suffisantes (à partir de $20 \mathrm{~cm}$ de diamètre pour le Mélèze, au moins le double pour les autres essences).

Biologiquement, ce sont des espèces qui répondent bien à l'offre d'habitats et qui en toute logique pourraient devenir plus abondantes et plus largement répandues dans nos montagnes avec une meilleure offre d'habitats favorables et davantage d'observateurs expérimentés. La fréquence des arbres favorables semble actuellement faible dans nos forêts car l'habitat reste éphémère et les forêts trop exploitées et exagérément "toilettées" donc trop jeunes et trop entretenues.

Les habitats occupés comprennent les habitats d'intérêt communautaires suivants:

- UE 9410 Forêts acidophiles à Picea des étages montagnards à alpin (Vaccinio-Piceetea);

- UE 9420 Forêts alpines à Larix decidua et/ou Pinus cembra; - UE 9530-2* pinèdes (sub) méditerranéennes de pins noirs endémiques: Pinus nigra subsp. laricio var. corsicana;

- Pinède basophile, méso- à xérophile des adrets et des ubacs à Pin à crochets (Pinus uncinata) habitat rattaché au UE 9430*.
Soit les habitats Corine Biotopes suivants: «CB 42.3 (bois de Mélèzes et d'Ayrolles) » (a priori le plus favorable); “CB 42.1 (Sapinières) » et "CB 42.5 (Pessières)». La tranche altitudinale 1100-2200 m concentre toutes les données liées à un développement larvaire avéré connues à ce jour en France.

Potentiellement, toutes les Alpes sont susceptibles d'accueillir ces deux espèces. Toutes les montagnes corses et l'est des Pyrénées pourraient aussi héberger Stephanopachys linearis. Ce sont ces zones, ces habitats et ces plages d'altitude qu'il faut délimiter, quantifier et ensuite prospecter davantage. Une modélisation de niche à partir de l'ensemble des données précises devrait permettre d'affiner l'enveloppe de présence potentielle de ces deux espèces.

Orientation pour la mise en place d'une surveillance nationale L'objectif est de mieux cerner la distribution de ces espèces et l'évolution de l'occupation du territoire, par une vérification périodique du maintien de l'espèce dans les stations connues (nombre de stations avérées, probabilité de détection, cartographie des surfaces favorables).

Les aléas à rencontrer des dendro-microhabitats favorables (occurrences rares et d'intérêt fugace dans le temps) obligent à expérimenter la création de dendro-microhabitats attractifs de ces espèces par génie écologique à des fins de détection et de suivi à long terme. La réalisation de ce protocole par échantillonnages à l'échelle d'un massif forestier pourrait également fournir des indications en termes de densité et d'occupation spatiale (capacité à saturer les niches offertes).

Le cahier des charges de création et le design de ces habitats artificiels est le suivant (Fig. 15):

- accord/participation du propriétaire/gestionnaire;

- choix d'au moins deux Mélèzes proches, de 20 à $50 \mathrm{~cm}$ de diamètre, si possible bien exposés et mal conformés; ces 


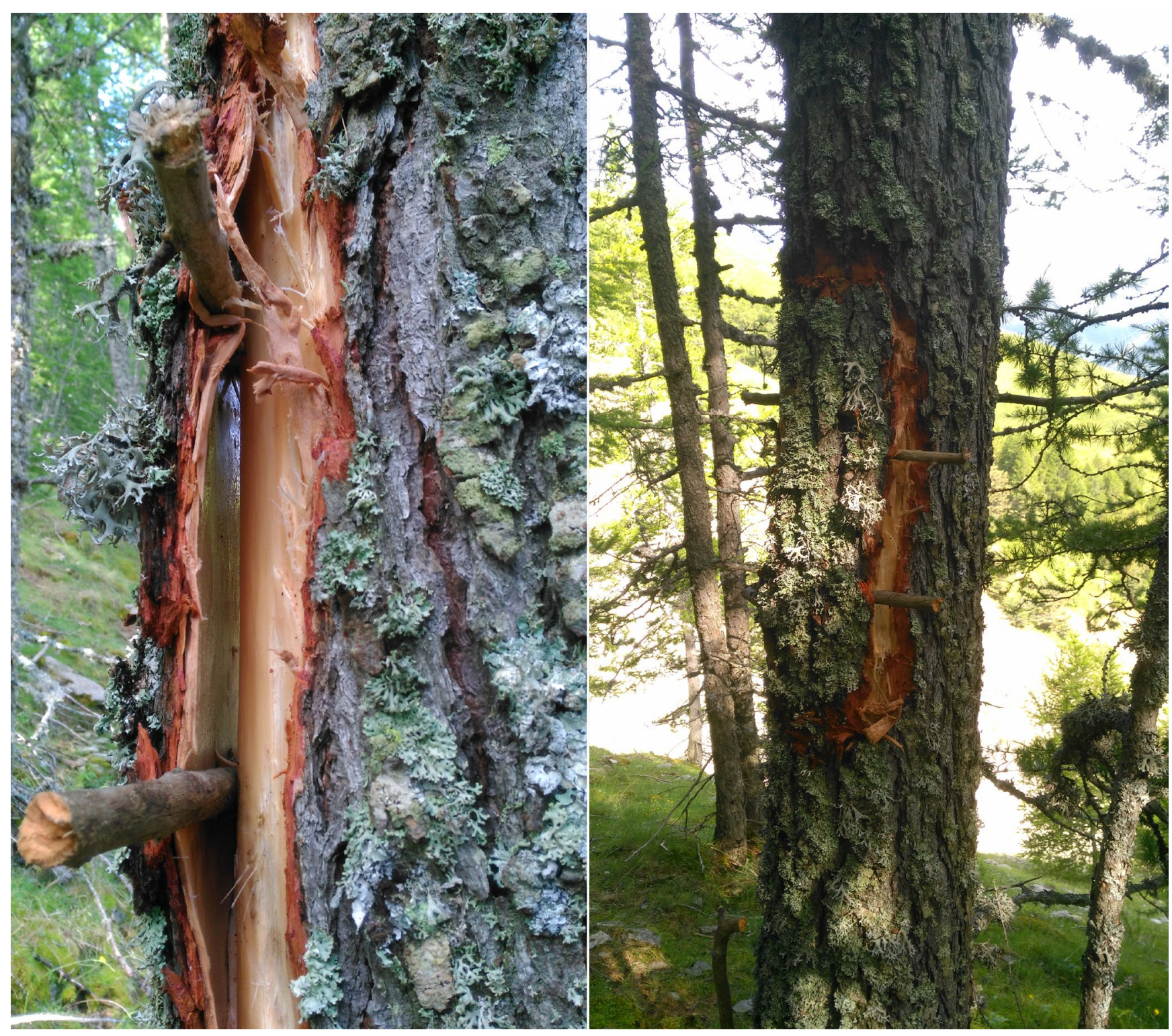

FIG. 15. - Dendromicrohabitat artificiel pour Stephanopachys spp. Crédit photos: Y. Braud.

espèces étant relativement mobiles, les arbres peuvent être choisis très accessibles pour l'opérateur;

- entailles longitudinales de $50 \mathrm{~cm}$ à hauteur d'homme pour créer une lanière de 20 à $25 \mathrm{~cm}$ de large, solidaire à l'arbre en haut et en bas mais démasclée sur toute sa hauteur et maintenue décollée par des bouts de bois (un par arbre échantillon).

La période idéale dans l'année, le délai de retour sur site pour contrôler l'éventuelle colonisation et la durée d'intérêt de l'habitat créé restent à optimiser par expérimentation sur un site favorable (par exemple dans le Queyras où les deux espèces sont connues).

Lors du contrôle des habitats artificiels (Fig. 16), on cherchera la présence d'adultes vivants ou de restes chitineux. À défaut, les éventuelles larves et nymphes pourront être prises en élevage pour identification ultérieure à l'état adulte.

À la façon finlandaise, il serait intéressant de conduire des expérimentations dans les Alpes françaises: des îlots d'arbres (<1ha chacun) coupés et brulés au sein de peuplements jeunes et en déficit de micro-habitats pour saproxyliques, ou des îlots d'arbres adultes, brulés ou pas, laissés après coupe rase de fin de cycle sylvicole en futaie régulière. Ces expériences boréales ont permis de revoir dans une certaine abondance les Stephanopachys qui avaient déserté depuis des années les peuplements exploités (Hyvärinen et al. 2006).

Pour le suivi à large échelle de l'habitat d'espèce, nous préconisons de s'appuyer sur les statistiques issues des inventaires standardisés de l'inventaire forestier national (Benest et al. 2016). Il s'agit d'analyser, dans l'aire de distribution favorable, l'évolution des surfaces des types d'habitats naturels que fréquentent ces espèces et d'extraire également des informations de structures pertinentes (densité d'arbres résineux morts sur pieds).

L'ensemble des recommandations est synthétisé dans le Tableau 7. 


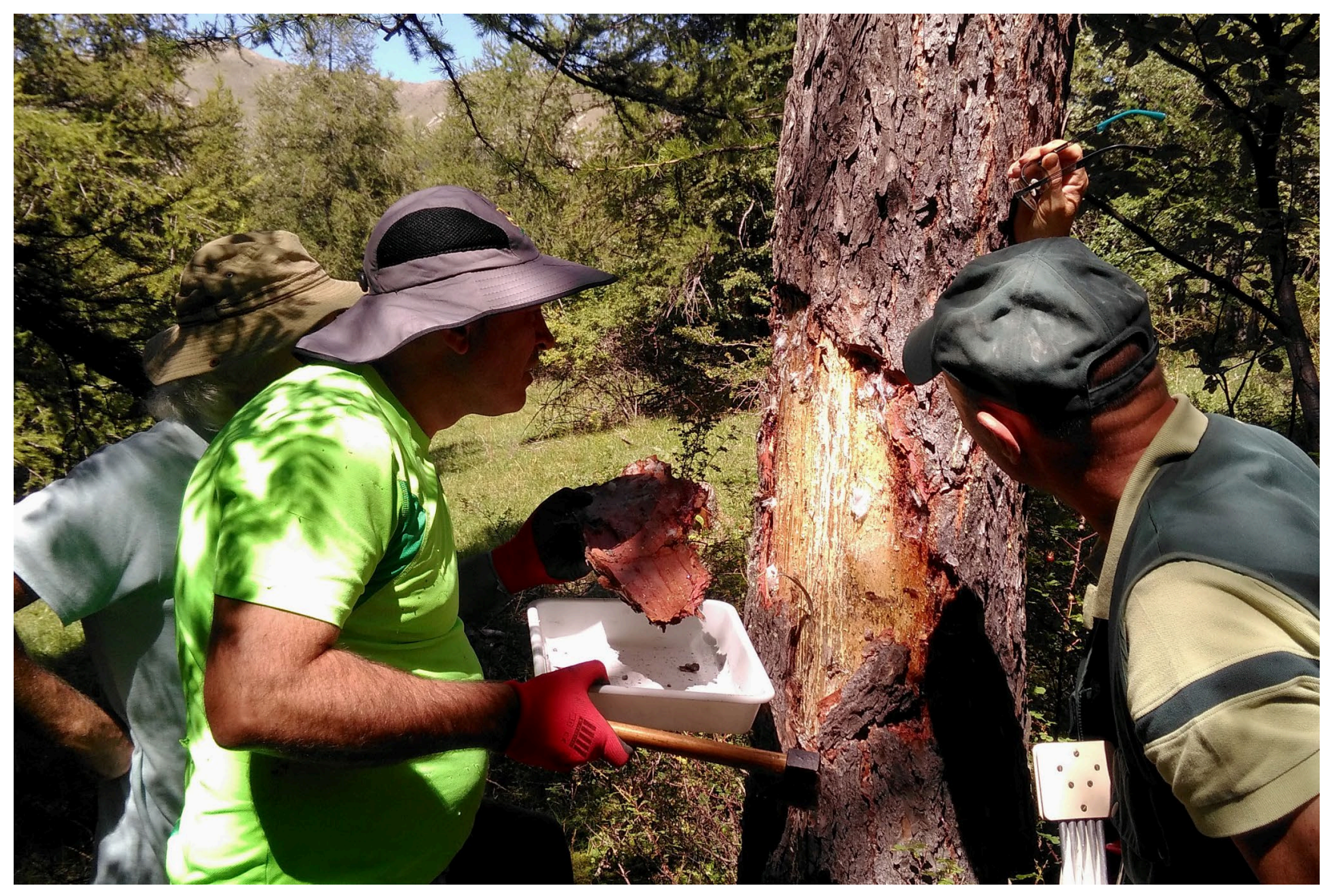

FIG. 16. - Contrôle d'un habitat artificiel sur Mélèze, deux ans après sa création (Saint-Martin-d'Entraunes, 06). Crédit photo: Y. Braud

\section{CERAMBYX CERDO (GRAND CAPRICORNE)}

Le Grand Capricorne du chêne (Fig. 17) est une espèce distribuée dans toute l'Europe, jusqu'au Moyen-Orient et au nord de l'Iran. En France, elle est potentiellement présente sur tout le territoire mais s'avère nettement plus commune dans le Sud, notamment en zone méditerranéenne. Elle est aujourd'hui considérée comme absente ou très localisée du Nord de la France avec quelques observations connues en Alsace et en Belgique (Berger 2012; Drumont et al. 2012). L'espèce se développe préférentiellement dans les vieux chênes dépérissant de gros diamètre et ensoleillés mais dans la région méditerranéenne, elle se développe sur des chênes de diamètres beaucoup plus faibles. On la trouverait également, beaucoup plus rarement, dans le Frêne (Acer negundo L.), le Saule (Salix alba L.) ou même le Châtaignier (Castanea sativa Mill.)(Sama 2002). La larve se développe dans le bois encore vivant (ou fraichement mort) durant au moins trois ans avant d'émerger durant l'été (pic du 20 juin au 10 août, d'après 5500 données disponibles dans l'INPN). C'est à ce moment qu'il est alors possible de détecter sa présence, soit par observation directe au crépuscule en vol ou sur l'écorce des arbres, soit par observation des traces larvaires et/ou d'émergence («trou de sortie») ou de débris d'adultes morts.

Cette espèce est relativement simple à détecter dans le sud de la France en raison de sa relative abondance, même si les trous de sorties dans le sud ont aussi plus de chances d'être d'autres espèces de Cerambycidae. En revanche, elle s'avère rare et localisée à des massifs forestiers avec de très vieux arbres ou des milieux ouverts avec de vieux arbres de tradition agropastorale ou de parcs arborés dans le nord et le centre de la France.

La réflexion autour de la mise en œuvre d'une surveillance nationale de l'espèce doit donc prendre en considération cette variabilité géographique quant à l'exigence de l'espèce envers son habitat et à sa rareté toute relative suivant les régions.

\section{Paramètres visés par les protocoles}

Détection de nouvelles stations et confirmation de présence. La détection du Grand Capricorne du chêne est assez simple par la reconnaissance de marques importantes infligées à l'arbre lors du développement larvaire et l'émergence de l'adulte. La recherche de "trous de sortie» (Fig. 17) caractéristiques de l'espèce constitue le moyen le plus simple pour détecter sa présence (Buse et al. 2007; Redolfi De Zan et al. 2017). La couleur claire beige-roussâtre des bords du trou d'émergence (grise après quelques mois) et la présence de sciure fraiche permettent également d'évaluer si l'émergence est de l'année en cours ou plus ancienne. Il est impossible de différencier un trou de Cerambyx cerdo des autres grandes espèces de 
TABLEAU 7. - Synthèse des protocoles recommandés pour une surveillance nationale des Stephanopachys spp.

\begin{tabular}{|c|c|}
\hline Éléments du protocole & Détection - distribution \\
\hline $\begin{array}{l}\text { 1. Paramètres visés par le protocole } \\
\text { et objectifs détaillés }\end{array}$ & $\begin{array}{l}\text { Établir et suivre la distribution: } \\
\text { Confirmation régulière des mailles } 10 \times 10 \mathrm{~km} \\
\text { occupées par l'espèce. } \\
\text { Recherche complémentaire à proximité des } \\
\text { mailles connues. } \\
\text { Recherche exploratoire au-delà des massifs } \\
\text { connus (opportuniste et ciblée en fonction } \\
\text { de la distribution probable obtenue par } \\
\text { modélisation). }\end{array}$ \\
\hline $\begin{array}{l}\text { 2. Plan d'échantillonnage national, } \\
\text { choix des sites/placettes }\end{array}$ & $\begin{array}{l}\text { Dans chaque maille de } 10 \times 10 \text { favorable } \\
\text { d'après le modèle et secteurs de présence } \\
\text { avérée: } \\
\text { 1) rechercher directement des individus sur des } \\
\text { résineux avec blessures d'exploitation ou } \\
\text { naturelles (chocs de roches ou chablis) et/ou, } \\
\text { 2) tenter une recherche standardisée par création } \\
\text { du micro-habitat. }\end{array}$ \\
\hline 3. Unité d'échantillonnage & $\begin{array}{l}\text { Maille } 10 \times 10 \mathrm{~km} \text { de présence avérée et de } \\
\text { présence potentielle. } \\
\text { 1) Examen de cinq résineux avec des blessures } \\
\text { 2) Création de micro-habitats sur deux Mélèzes } \\
\text { (Larix decidua Mill.). }\end{array}$ \\
\hline 4. Techniques et matériel & $\begin{array}{l}\text { 1) Observation visuelle à l'aide d'un piochon et } \\
\text { bac } \\
\text { 2) Décollement d'écorce vivante en lanière d'env. } \\
20-25 \mathrm{~cm} \text { de large sur } 50 \mathrm{~cm} \text { de haut, au } \\
\text { niveau de l'assise subérophéllodermique. } \\
\text { À l'aide du piochon (éventuellement hache). }\end{array}$ \\
\hline
\end{tabular}

\section{Période(s) favorable(s)}

6. Variables et co-variables à relever

7. Technicité/compétence pour effectuer les relevés

8. Possibilité de mobiliser des opérateurs gestionnaires?

9. Périodicité des relevés

10. Type d'indicateur et d'analyse

11. Éléments de coût

12. Aspect expérimental à développer

13. Sites pilotes potentiels
Probablement toute l'année.

Par sécurité, mai-août. Pour la création de micro-habitats: année n-2 (printemps); passage année $n$ : avril-août.

Variables de structure: présence de coupes récente induisant blessures en bord de pistes ( $<5$ ans) et leur distance; présence de zones incendiées et leur distance.

Recherche active: expert. Installation d'habitats artificiels et relevé: simple mais détermination délicate (ressemblance avec Scolytinae); un gestionnaire s'il collecte tous les Coléoptères sous l'écorce et met l'ensemble en tube d'alcool. La mise en élevage de larves est plus délicate.

Possibilité de mobiliser les gestionnaires pour Valorisation des cartographies d'habitats. créer les habitats: explication sur photo.

ONF et gestionnaires Natura 2000.

Échantillonnage réparti sur un pas de temps d'environ 10 ans (pour les sites connus et mailles proches)

Taux d'occupation des habitats.

Nombre moyen de spécimens par habitat occupé.

Nombre et taux de maille où l'espèce est détectée dans l'aire favorable.

Matériel: faible (piochon, bac); installation 0,5 jour par maille (tenant compte déplacement, recherche mélézin, etc.); contrôle 0,5 jour par maille.

Traitement $=30$ min par maille.

Création d'habitats variés (essences, tailles, exposition, brulage dirigé, etc.), à l'échelle de quelques arbres ou de petits patchs, puis bilan de présence les années $n+1, n+2$, voire $n+3$. Modèles pour expliquer la présence en fonction de variables paysagères.

Queyras prioritairement (présence des deux espèces) et autres massifs où les Stephanopachys sont connus. Toute l'année.

Voir dans les statistiques forestières les surfaces incendiées ou autres perturbations (tempêtes, etc.) dans les mélézins et leur date.

mpétences fortes en traitement modélisation.

Actualisation des données IGN = 10 ans modélisations à actualiser avec les nouvelles données d'observations (tous les cinq ans).

Évolution de la surface d'habitats favorables.

Évolution de la densité moyenne de chandelles résineuses dans les habitats favorables.

Pour actualiser les enveloppes de distribution et statistiques sur les peuplements: deux mois d'un biostatisticien (tous les cinq ans).

\section{Suivi d'habitat - population}

Suivre la quantité et la qualité d'habitat. Analyse de données d'inventaire forestie (IGN) et modélisation pour connaître

- la surface potentiellement occupée:

- la surface totale favorable;

- la densité de chandelles de résineux dans les surfaces favorables.

\section{Modélisation et données d'inventaires}

forestiers existantes (selon grille d'inventaire IGN).

des chandelles, utilisation des données "bois mort » sur pied des placettes régulières du protocole d'inventaire forestier national de l'IGN.

Les sylvoécorégions dans l'enveloppe de distribution de l'espèce.

Traitement SIG et modélisation statistique: Macro-habitat favorable prioritaire: Mélézin + climat/altitude + restriction au Sud-Est de la France (+ buffer autour des stations connues)

cocro-habitat favorable secondaire: resineux (Pin sylvestre (Pinus sylvestris L.), Épicéa (Picea abies (L.) H.Karst.), Sapin pectiné (Abies alba Mill.)) + climat/altitude. 


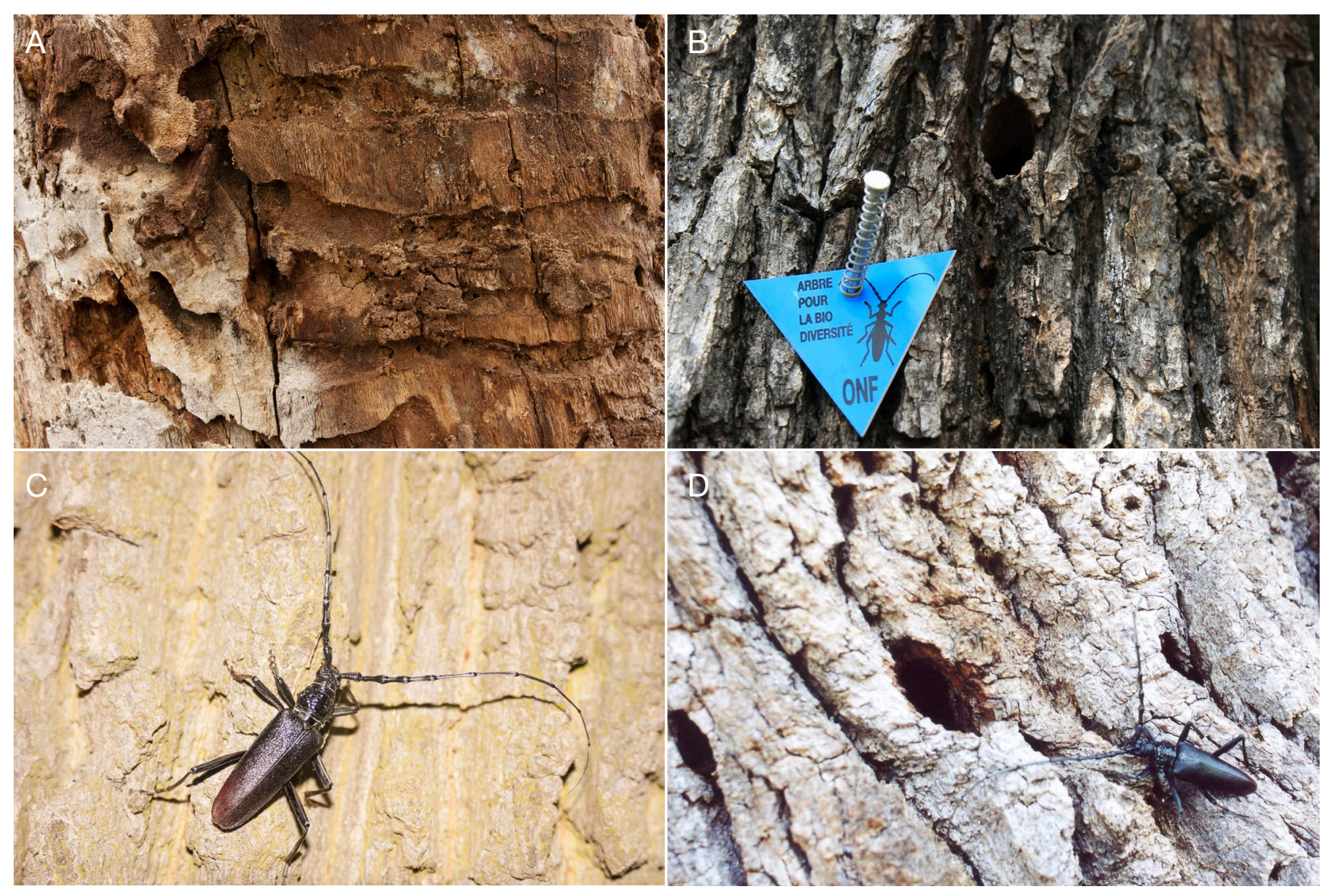

FIG. 17. - A, Tronc de chêne attaqué par les larves de Cerambyx cerdo Linnaeus, 1758; B, marquage d'un arbre conservé pour la biodiversité; C, mâle sur un tronc de chêne; $\mathbf{D}$, idem à proximité d'un orifice d'émergence. Crédits photos: N. Gouix (A,B et C) et L. Valladares (D).

Cerambyx (Cerambyx welensii (Küster, 1845); Cerambyx miles Bonelli, 1812) et difficile parfois avec Prinobius myardi Mulsant, 1842 ou Aegosoma scabricorne (Scopoli, 1763). Ces autres espèces peuvent d'ailleurs partager le même arbre que C. cerdo, notamment dans le Sud de la France et en Corse. D'un point de vue de la conservation, ces espèces présentent un intérêt patrimonial et une fonction écologiques équivalents (voire supérieurs) à $C$. cerdo et peuvent être inventoriées au même titre et les risques de confusion ne faussent pas le diagnostic patrimonial.

Pour valider la présence de l'espèce sur des secteurs où des trous de sortie ont été observés, nous conseillons donc la recherche d'imagos en début de nuit directement sur les troncs d'arbre ou la recherche de débris d'imagos le long de transects durant la période d'émergence, à savoir entre fin juin et fin août (Redolfi De Zan et al. 2017).

Dans les secteurs où l'espèce est rare (ou discrète), il peut toutefois s'avérer difficile de découvrir l'arbre avec un ou deux trous de sortie de l'espèce parmi l'ensemble d'un boisement. L'utilisation de technique de piégeage attractive est alors pertinente pour capturer l'imago. Le "piège à bière (ou à vin)» disposé dans les arbres est certainement le piège le plus largement utilisé par les entomologistes. Il s'avère particulièrement pertinent pour la capture de Cerambyx cerdo. L'attractif le plus efficace pour l'espèce se compose d'un mélange de bière ou de vin, de banane et de sucre (odeur de fermentation riche en alcools et acétates, à la manière d'une plaie suintante d'arbre; Döring 1955). Si l'efficacité de ce type de piège n'est plus à démontrer, son inconvénient est qu'un individu attiré se noie dans le dispositif si une grille n'est pas disposée en protection. La présence d'une grille de protection permet de sauver les individus collectés à condition que le piège soit contrôlé régulièrement (maximum tous les trois jours, idéalement tous les jours pour éviter les mutilations) pour relâcher les individus capturés.

Les conditions d'utilisation optimale de ce type de piège pour la capture de Cerambyx cerdo sont précisées par Redolfi De Zan et al. (2017). Pour un site donné, ils conseillent la mise en place de 20 pièges sur 10 arbres (1 à environ 1,5-2 $\mathrm{m}$ de haut et l'autre à environ $10 \mathrm{~m}$ de haut) en conservant une distance minimale de $100 \mathrm{~m}$ entre chaque arbre. La période de piégeage peut se restreindre aux cinq semaines les plus favorables (juin-juillet) à raison de 15 relèves sur la période. La réussite de ce dispositif sera augmentée par la sélection des arbres les plus favorables du site, et une exposition ensoleillée des pièges.

Les observatoires participatifs, en particulier ceux qui associent une photographie à l'observation (i-Naturalist, INPN-Espèces), peuvent apporter de nombreuses données 


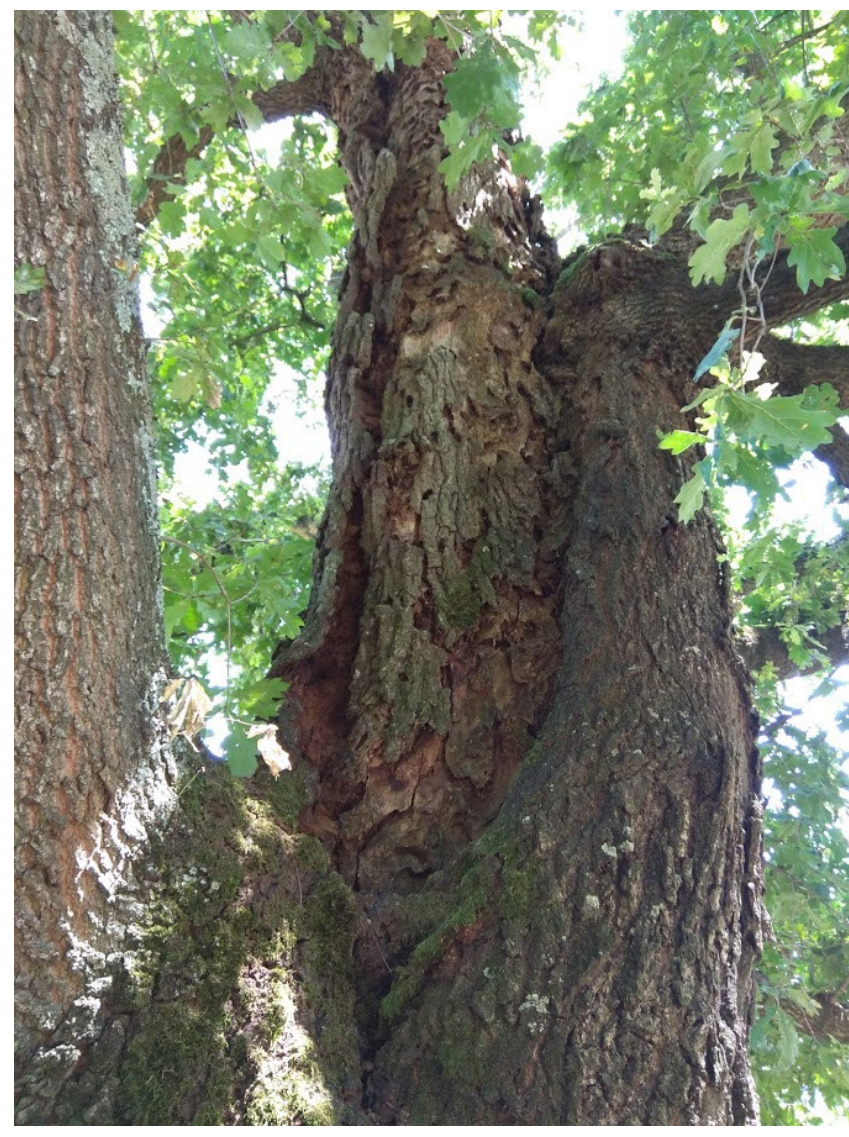

FIG. 18. - Tronc de chêne colonisé par Cerambyx cerdo Linnaeus, 1758, traces caractéristiques de galeries sous l'écorce. Crédit photo: L. Valladares.

de distribution et sont une source à considérer pour cette espèce. Les photographies des critères de reconnaissance morphologique sont particulièrement importantes (confusions fréquentes avec les deux autres Cerambyx de grande taille dans le Sud).

Suivi des habitats. Pour les sites où l'espèce est connue, suivre la ressource en arbres favorables permet à partir d'un état initial de projeter une évolution en habitats disponibles. Les besoins écologiques de l'espèce ont été particulièrement étudiés en Allemagne (Buse et al. 2007, 2008). Ces résultats montrent que les vieux arbres déficients et bien exposés au soleil sont particulièrement favorables à l'espèce (Fig. 18). Le diamètre de l'arbre est un autre facteur mis en avant dans la probabilité de capturer l'espèce (Redolfi De Zan et al. 2017). Dans les sites où l'on veut suivre l'évolution de la ressource en habitat disponible pour le Grand Capricorne, il est donc recommandé de repérer l'ensemble des arbres de gros diamètre (diamètre de plus de $50 \mathrm{~cm}$, sauf en chênaie verte où $30 \mathrm{~cm}$ est suffisant) et de caractériser leur vitalité et leur ensoleillement selon les critères de Buse et al. (2007). La présence de trous de sortie permet sur cette ressource de valider le nombre d'arbres occupés et le nombre d'arbres potentiels sur un site. Tout gros chêne qui présente des signes de dépérissement (descente de cime, exsudats sur le tronc) peut être déjà occupé par Cerambyx cerdo.
TABLEAU 8. - Proposition de critères à suivre pour évaluer la potentialité d'accueil des arbres favorables au Grand Capricorne (Cerambyx cerdo Linnaeus, 1758), en dehors de la région méditerranéenne.

\begin{tabular}{llll}
\hline $\begin{array}{l}\text { Diamètre de } \\
\text { l'arbre }\end{array}$ & Vitalité & Ensoleillement & $\begin{array}{c}\text { Présence de } \\
\text { trou de sortie }\end{array}$ \\
\hline 1: $\mathrm{DBH}<50$ & 1: Arbre vivant & 1: Ombre & 1: Oui \\
2: $0<\mathrm{DBH}<100$ & 2 : Arbre & 2: Partiellement $2:$ Non \\
dépérissant & $\begin{array}{c}\text { ensoleillé } \\
\text { 3: } \mathrm{DBH}>100\end{array}$ & $\begin{array}{l}\text { 3: Arbre mort } \\
\text { 3: Totalement } \\
\text { ensoleillé }\end{array}$ \\
\hline
\end{tabular}

Aucun suivi à l'échelle nationale n'a encore été conduit en suivant ces critères (Tableau 8). La proposition de relever ces paramètres par tous les opérateurs entomologistes ou gestionnaires de sites étudiant le Grand Capricorne pourrait permettre rapidement de créer une "règle de décision " simple comme celle élaborée pour Limoniscus violaceus (Gouix 2011).

Si ce schéma est particulièrement vrai dans le Nord et le centre de la France, il est à nuancer dans le Sud où des arbres de petit diamètre en contexte peu ensoleillé sont régulièrement occupés par l'espèce. Si l'espèce est plus facile à détecter en raison d'une plus grande abondance au fur à mesure que l'on se rapproche du contexte méditerranéen, la mise en place d'un suivi d'habitat devient elle plus compliquée par la quantité de milieux potentiellement favorables. Dans ce contexte particulier, les statistiques forestières nationales (inventaire forestier de l'IGN, Benest et al. 2016) devraient permettre d'obtenir des informations sur la surface et la structure de l'habitat de cette espèce en zone méditerranéenne.

Orientations pour la mise en place d'une surveillance nationale La mise en place d'une surveillance nationale de Cerambyx cerdo poursuit deux objectifs principaux (Tableau 9):

- affiner notre connaissance des sites où l'espèce est présente, ce qui passe par la détection de nouveaux sites avérés et des secteurs de présence potentielle, et la confirmation périodique (10 ans) de la présence sur les sites connus;

- suivre les habitats présents et en devenir de l'espèce pour leur prise en considération dans la gestion et pour mesurer des tendances d'évolution.

Compte tenu de la large distribution de l'espèce et des menaces qui pèsent sur les vieux arbres, c'est l'aspect suivi des habitats qui apparaît comme prioritaire.

La distribution sera approchée par modélisation dans la partie sud de son aire de répartition (climat + bocage ou chênaie). De nouvelles stations seront aussi obtenue par les observatoires naturalistes (entomologistes et grand public, sur photo: Casula 2017), ainsi que par le partage des données d'étude d'impact dans le SINP. Dans les secteurs où l'espèce est plus abondante (Sud de la France) une enquête participative sur la détection d'arbres avec trous de sortie "où l'on peut passer deux bouts de doigts " pourrait être envisagée.

La recherche de nouveaux sites par la mise en œuvre d'un protocole de détection doit être limitée et priorisée sur le tiers nord de la France, en ciblant des vieux peuplements forestiers et les secteurs de vieux arbres feuillus de type bocage, alignements, 
TABLEAU 9. - Synthèse des protocoles recommandés pour une surveillance nationale de Cerambyx cerdo Linnaeus, 1758. Abréviations: G, Global, c'est-à-dire concernant toutes les populations; MED, région biogéographique méditerranéenne; N, Nord, à savoir le reste de la France; SO, Sud-Ouest (jusqu'à la Loire).

\begin{tabular}{|c|c|c|}
\hline Éléments du protocole & Détection - distribution & Suivi d'habitat - population \\
\hline $\begin{array}{l}\text { 1. Paramètres visés par le protocole et } \\
\text { objectifs détaillés }\end{array}$ & $\begin{array}{l}\text { G: Mieux définir la distribution de l'espèce et } \\
\text { suivre cette distribution dans le temps. } \\
\mathrm{N} \text { : identifier de nouvelles stations. } \\
\mathrm{N} \text { : confirmer régulièrement le maintien de } \\
\text { l'espèce. }\end{array}$ & $\begin{array}{l}\text { G: Suivre les surfaces d'habitat potentiel et } \\
\text { les caractéristiques de structure favorables } \\
\text { à l'espèce (variable selon les régions). }\end{array}$ \\
\hline $\begin{array}{l}\text { 2. Plan d'échantillonnage national, choix des } \\
\text { sites/placettes }\end{array}$ & $\begin{array}{l}\text { G: non structuré, sur tout le territoire, } \\
\text { modélisation de la distribution probable } \\
\text { en utilisant l'ensemble des données } \\
\text { disponibles; } \\
\mathrm{N} \text { : échantillonnage aléatoire des sites connus } \\
\text { (par ex. } 50 \% \text { des sites pour le domaine } \\
\text { continental). }\end{array}$ & $\begin{array}{l}\text { N: échantillonnage aléatoire des sites connus } \\
\text { (par ex. } 50 \% \text { des sites pour le domaine } \\
\text { continental). } \\
\text { MED, SO: plan d'échantillonnage de } \\
\text { l'inventaire forestier national (grille de } \\
1 \times 1 \mathrm{~km} \text { ). } \\
\text { SO: tirage aléatoire de } 50 \text { ZNIEFF } \\
\text { mentionnant l'espèce. }\end{array}$ \\
\hline 3. Unité d'échantillonnage & $\begin{array}{l}\text { G: France entière (modélisation, données } \\
\text { opportunistes). } \\
\mathrm{N} \text { : la station, permanente (ensemble de } \\
\text { vieux chênes séparés par moins de } 500 \mathrm{~m} \\
\text { les uns des autres). }\end{array}$ & $\begin{array}{l}\text { N: la station, permanente (cf. colonne } \\
\text { détection). } \\
\text { MED, SO: placettes (non permanentes) } \\
\text { de l'inventaire forestier national, dans la } \\
\text { distribution et avec du chêne. } \\
\text { SO: placette permanente de } 50 \text { chênes } \\
\text { proches (tous diamètres). }\end{array}$ \\
\hline 4. Techniques et matériel & $\begin{array}{l}\text { G: outils de saisie, observatoires, bases de } \\
\text { données SINP. } \\
\text { N: observation visuelle de macro-restes } \\
\text { et trous d'émergence; éventuellement } \\
\text { piégeage ciblé ( } 20 \text { pièges). }\end{array}$ & $\begin{array}{l}\text { N, SO: Observation visuelle des arbres } \\
\text { (observation opportuniste des macro-restes } \\
\text { et individus actifs). } \\
\text { MED: réalisé par l'IGN. }\end{array}$ \\
\hline 5. Période(s) favorable(s) & $\begin{array}{l}\text { G: pic du } 20 \text { juin au } 10 \text { août pour observer } \\
\text { l'adulte. } \\
\text { N: juillet-septembre pour les macro-restes; } \\
10 \text { juillet-10 août pour le piégeage. }\end{array}$ & Toute l'année. Possible en hiver \\
\hline 6. Variables et co-variables à relever & $\begin{array}{l}\text { N: position de l'arbre, essence, diamètre, } \\
\text { éclairement, photo du macro-reste } \\
\text { Noter les autres espèces observées } \\
\text { (Lucanus, Dorcus, Aegosoma, cétoines } \\
\text { etc.). }\end{array}$ & $\begin{array}{l}\mathrm{N} \text { : position de l'arbre, essence, diamètre, } \\
\text { éclairement. } \\
\text { Noter et photographier les macro-restes et } \\
\text { autres espèces observées (opportuniste). }\end{array}$ \\
\hline $\begin{array}{l}\text { 7. Technicité/compétence pour effectuer } \\
\text { les relevés }\end{array}$ & $\begin{array}{l}\text { G: forte compétence en traitement de } \\
\text { données. } \\
\text { N: Technicité pour les reconnaissances } \\
\text { des macro-restes et la reconnaissance } \\
\text { des arbres favorables (une journée de } \\
\text { formation); pose de pièges simples. }\end{array}$ & $\begin{array}{l}\text { MED: forte compétence en traitement de } \\
\text { données. } \\
\mathrm{N}, \mathrm{SO}: \text { Technicité d'écologue pour les critères } \\
\text { à noter. }\end{array}$ \\
\hline $\begin{array}{l}\text { 8. Possibilité de mobiliser des opérateurs } \\
\text { gestionnaires? }\end{array}$ & Oui & Oui \\
\hline 9. Périodicité des relevés & $\begin{array}{l}\text { G: sans objet. Analyse tous les six ans. } \\
\mathrm{N} \text { : détection tous les } 10 \text { ans. } \\
\text { Si piégeage, relève tous les trois jours. }\end{array}$ & $\begin{array}{l}\text { MED: sans objet. Analyse tous les six ans. } \\
\mathrm{N}, \mathrm{SO} \text { : suivi des stations et placettes tous les } \\
\text { cinq ans. }\end{array}$ \\
\hline 10. Type d'indicateur et d'analyse & $\begin{array}{l}\text { G: Nombre de stations, nombre de mailles } \\
\text { connues (avant données }<10 \text { ans), surface } \\
\text { favorable d'après le modèle. } \\
N \text { : nombre d'arbres colonisés, taux d'arbres } \\
\text { favorables par station. }\end{array}$ & $\begin{array}{l}\text { MED: surface de chênaies, densité de tiges } \\
>30 \mathrm{~cm} \text {; SO idem mais diamètre }>60 \mathrm{~cm} \\
\text { et } 80 \mathrm{~cm} \text {. } \\
\text { N, SO: évolution de la densité d'arbres } \\
\text { favorables (Tableau 8). }\end{array}$ \\
\hline 11. Éléments de coût & $\begin{array}{l}\text { Animation du SINP et des programmes } \\
\text { participatifs avec photo (déjà existants). } \\
\text { Traitement et analyse de données } \\
\text { périodiquement. }\end{array}$ & $\begin{array}{l}\text { MED, SO: temps d'analyse de données. } \\
\mathrm{N}, \mathrm{SO}: 0,5 \text { jour par station/placette } \\
\text { (cheminement compris). }\end{array}$ \\
\hline 12. Aspect expérimental à développer & $\begin{array}{l}\text { Capacité de déplacement selon la } \\
\text { température. Estimer les densités } \\
\text { d'individus dans les différents types } \\
\text { d'habitats; tester les règles de notation de } \\
\text { l'arbre permettant d'estimer la probabilité } \\
\text { de présence de l'espèce. }\end{array}$ & \\
\hline 13. Sites pilotes potentiels & $\begin{array}{l}\text { Privilégier des sites suivis pour les autres } \\
\text { espèces. }\end{array}$ & \\
\hline
\end{tabular}


etc. Au niveau local, la détection doit aussi concerner des sites protégés qui abritent certainement l'espèce sans que cette information ne soit connue et prise en compte par le gestionnaire.

Confirmer la présence de l'espèce sur des sites connus est plus simple à organiser. Une vérification tous les 10 ans semble suffisante considérant les vitesses d'évolution des vieux arbres. Cela est facilement réalisable par des opérateurs non entomologistes après une formation succincte.

Suivi de l'habitat. Le suivi de l'habitat peut concerner tous les sites où l'espèce est avérée avec une logique d'échantillonnage. Il repose sur la caractérisation des arbres potentiels et des arbres occupés par l'espèce.

Dans les milieux ouverts ou semi-ouverts (bocage, « devèze», "plantades», etc.), le travail de repérage des gros arbres favorables à l'espèce est plus facile. Par exemple, sur tracé routier d'environ $10 \mathrm{~km}$, près de 200 arbres fortement potentiels ou occupés par l'espèce ont ainsi pu être marqués en trois jours de terrain (Gouix obs. pers). Pour comparaison, le repérage d'un nombre d'arbre équivalent dans un boisement de 70 ha a pris environ cinq jours de terrain pour un observateur expérimenté (Gouix obs. pers).

Dans le tiers nord de la France où l'espèce est rare et peu abondante le suivi d'une majorité des sites peut être envisagé. Dans le cadre d'un suivi mis au point en réponse à une logique de gestion, suivre l'évolution de l'habitat de l'espèce tous les cinq ans semble pertinent. L'effort nécessaire pour la réalisation de ce type de suivi sera variable en fonction de la taille du site mais on peut considérer un temps compris entre deux et six jours comme satisfaisant, sachant qu'un système de placette de suivi peut être mis en place pour les sites les plus grands.

Dans le Sud de la France, l'exercice est plus compliqué en raison du nombre d'arbres potentiels important. Dans ce cas, on peut envisager un tirage aléatoire de 50 sites par région biogéographique et la réalisation de placettes représentatives du peuplement (une placette pourrait être définie comme 50 chênes de $50 \mathrm{~cm}$ de diamètre et plus). Cette approche est pertinente pour les secteurs hors forêt (Sud-Ouest de la France).

L'autre option, que nous recommandons pour la zone méditerranéenne, serait d'utiliser les statistiques forestières de l'IGN (Benest et al. 2016), concernant les surfaces de chênaie (chênes vert, pubescent, tauzin, sessile et pédonculé) intersectées avec la distribution de l'espèce (modélisée ou avérée) et de calculer des variables de structure comme la densité de gros et très gros bois vivant à l'hectare. Pour les arbres de bocage et arbres isolés en milieu agricole, les données de l'enquête de Teruti-Lucas du Service de la Statistique et de la Prospective (SSP) du ministère en charge de l'agriculture croisées avec la distribution de l'espèce, permettraient une quantification des éléments favorables et de leur évolution (en s'inspirant de l'indicateur de l'observatoire national de la biodiversité: http:// indicateurs-biodiversite.naturefrance. $\mathrm{fr} / \mathrm{fr} /$ indicateurs/ haies-bois-et-landes-dans-les-territoires-agricoles, dernière consultation le 14 août 2019).
Rosalia alpina (Rosalie Des Alpes)

La Rosalie des Alpes est une espèce de longicorne xylophage, spectaculaire par sa taille et surtout sa coloration, et ainsi très simple à reconnaître (Fig. 19). Sa larve consomme du bois mort en cours de dégradation. L'espèce est principalement montagnarde et liée au Hêtre dans ce contexte (Russo et al. 2011). En plaine, on la retrouve principalement sur les feuillus de ripisylve et sur le Frêne (Cizek et al. 2009; Berger 2012). Considéré souvent comme un habitat de substitution, les enquêtes participatives (Rabinovitch et al. 2017) montrent que la distribution en plaine (façade atlantique en particulier) s'avère beaucoup plus dense et étendue qu'on ne le croyait jusqu'à maintenant.

Drag et al. (2011) ont étudié les capacités de déplacement de l'espèce et démontré une mobilité importante (plus de $1,6 \mathrm{~km}$ ). Ils mettent toutefois en évidence la nécessité de conserver des îlots de vieux Hêtres (îlots de sénescence) en condition ensoleillée pour préserver les populations de l'espèce sur le long terme.

Conserver des vieux Hêtres sur pied dans les peuplements au cours des martelages est utile pour maintenir les connexions entre les différentes populations. Le choix peut s'orienter en priorité sur des arbres présentant des «défauts» pour le forestier mais un fort intérêt pour la biodiversité.

En forêt de Hêtres, une des principales sources de diminution des effectifs de Rosalie des Alpes résiderait dans l'exportation des pontes (Adamski et al. 2016, 2018). Les arbres ou tas de bois stockés en bordure de routes en été sont très attractifs et de très nombreuses femelles viennent y pondre. Ces bois partent en scierie emportant avec eux une partie non négligeable des œufs et larves de l'espèce ainsi que de nombreux insectes. Dans les zones à Rosalie des Alpes, le Hêtre (grume et bois de chauffage) doit être enlevé avant le 1er juillet pour éviter ce phénomène (Noblecourt 2005). Pour les forêts bénéficiant du Régime forestier, il existe à l'Office National des Forêts, une prescription nationale concernant la Rosalie des Alpes et libellée comme suit: "Tous travaux interdits du 15/06 au 15/08. Enlever en forêt et sur places de dépôt tous produits de Hêtre avant le 15/06». Elle concerne les exploitations et est appliquée dès que la présence de Rosalie des Alpes sur le secteur est avérée.

\section{Paramètres visés par les protocoles}

Détection de nouvelles stations et confirmation de présence. Les populations de cette espèce n'ont été que récemment étudiées malgré son caractère remarquable et protégé. Une étude démontre une taille de population importante ( 42 à 84 adultes par hectare et par an) et met en évidence d'importantes capacités de dispersion (plus de 1,6 km) (Drag et al. 2011). Ces résultats expliquent les abondances importantes qui peuvent être observées pour cette espèce en montagne dans les vieilles hêtraies lorsque l'offre d'habitat est importante et le rétablissement des populations en Suisse suite à une meilleure prise en compte des stades forestiers âgés (Lachat et al. 2013). En plaine, les abondances restent faibles et les observations sporadiques. Certains auteurs l'imputent à une expansion de l'espèce dans un modèle écologique moins favorable à 


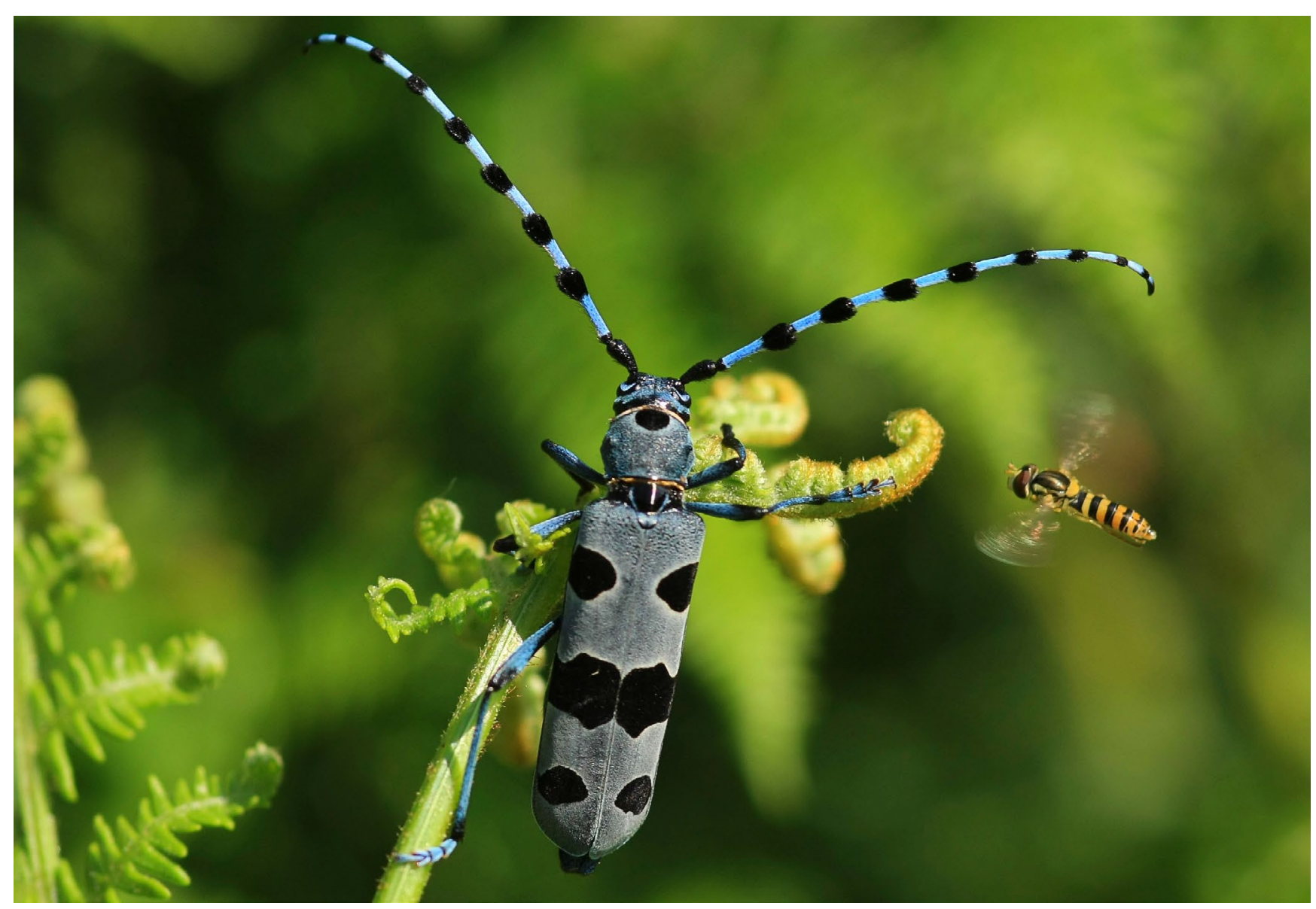

FIG. 19. - La Rosalie des Alpes, Rosalia alpina (Linnaeus, 1758), un Coléoptère qui peut être reconnu par un large public. Crédit photo: N. Gouix.

sa présence (Cizek et al. 2009; Michalcewicz et al. 2011; Michalcewicz \& Ciach 2012). Ce point mériterait d'être étudié en France, où les populations de plaine semblent discrètes mais largement distribuées.

Les adultes vivent quelques semaines tout au plus et se rencontrent de fin juin - début juillet à septembre. On les observe en général sur les très vieux arbres avec de grosses branches mortes (Fig. 20), les chandelles, les tas de grumes et sur les tas de bois de chauffages. Berger (2012) précise que cette espèce aime la chaleur et le soleil.

Dans le cadre de prospections, il convient donc de rechercher les vieux arbres et en particulier en montagne, les tas de grumes de Hêtres stockées au soleil.

Dans les secteurs favorables, des indices de présence (trous de sortie, cadavres, larves) peuvent être recherchés, bien que cette technique ne soit pas évidente pour cette espèce. Le trou de sortie de la Rosalie est assez caractéristique. Il présente une forme aplatie, en général orientée dans le sens des fibres du bois et perpendiculaire à l'axe du tronc. Il est en outre d'assez grande taille $(10 \mathrm{~mm})$ et les galeries de cette espèce sont remplies de sciure. Ils peuvent toutefois être confondus avec ceux de Cerambyx scopolii Fuessly, 1775 (dont les trous sont semblables mais dont la galerie est oblique par rapport à la surface du tronc) voire de grosses leptures ou Rhagium mordax (De Geer, 1775).
En montagne, la recherche d'adultes sur les grumes coupées et sur les troncs des chandelles peut être réalisée aux heures les plus chaudes de la journée et préférentiellement dans les situations bien exposées au soleil, par exemple en contextes forestiers semi-ouverts et en clairières où les bois morts seront préférentiellement colonisés (Russo et al. 2011 ; Berger 2012; Castro \& Fernández 2016).

En plaine, pour valider la présence de l'espèce sur des zones de ripisylves, la mise en place de piège attractif non destructeur durant la période d'émergence, à savoir entre début juillet et mi-août (Sama 2002) peut être envisagée. Le "piège à bière» disposé dans les arbres est certainement le piège le plus largement utilisé par les entomologistes. Il s'avère particulièrement pertinent pour la capture de Rosalia alpina dans les mêmes conditions d'utilisation que pour Cerambyx cerdo.

Comme pour le Lucane cerf-volant, Zapponi et al. (2017) ont démontré tout l'intérêt des enquêtes participatives pour préciser rapidement la distribution de cette espèce. Il s'agit en effet d'une espèce que tout citoyen peut remarquer et photographier avec un smartphone pour ensuite la transmettre dans une enquête dédiée ou via un outil de signalement participatif (i-Naturalist et INPN-Espèces par exemple). En France, l'enquête menée depuis 2014 par l'Opie et le GRETIA a rapidement apporté de nombreuses localités nouvelles et confirmé des sites connus. 


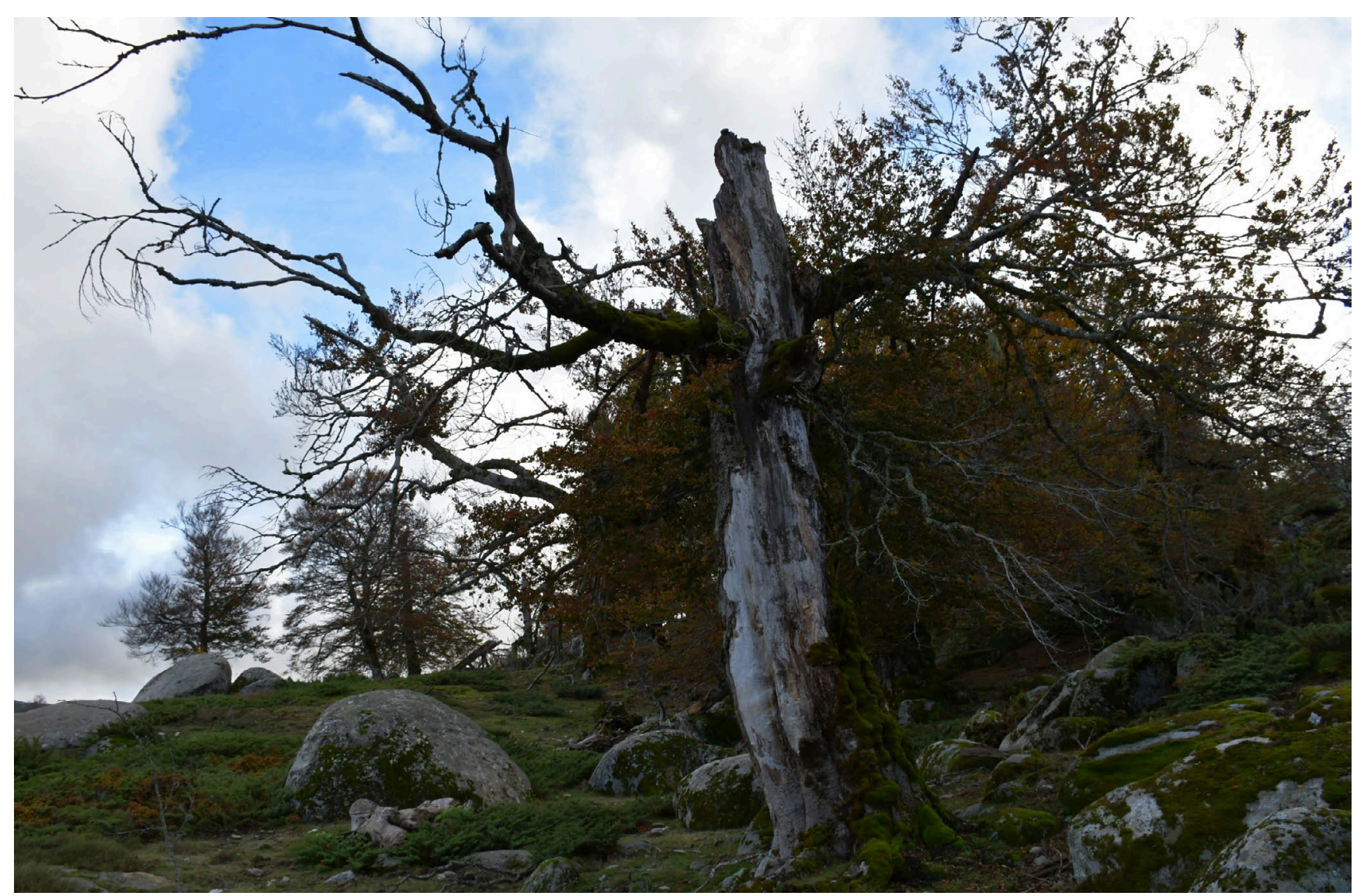

FIG. 20. - Hêtraie de montagne, habitat de Rosalia alpina (Linnaeus, 1758) en Corse. Crédit photo: J. Touroult.

Suivi des habitats. Pour les sites où l'espèce est connue, suivre la ressource en arbres favorables à l'espèce permet de dégager à partir d'un état initial une évolution en habitat disponible sur un site (Tableau 10).

En plaine, c'est l'évolution de l'état de conservation des corridors écologiques, des haies bocagères et des ripisylves en particuliers qu'il faut suivre.

En montagne, il s'agit de suivre le maintien de la hêtraie avec du bois mort, en particulier des chandelles, et également le niveau d'ouverture des peuplements.

\section{Orientations pour la mise en place d'une surveillance nationale} Pour la distribution, les remontées par les programmes participatifs nous semblent de loin la meilleure façon de trouver de nouvelles stations et de confirmer les stations connues. La recherche ciblée par des entomologistes ne devra s'envisager que dans le cadre d'un enjeu local comme la confirmation de présence dans un espace protégé.

Concernant le suivi d'habitat, les relevés statistiques de l'inventaire forestier national (Benest et al. 2016) devraient permettre de connaître l'évolution des habitats naturels utilisés par l'espèce.

En montagne, il s'agit de calculer et suivre la surface d'habitats naturels de hêtraies dans la zone de présence de la Rosalie des Alpes et de suivre la densité de gros bois morts sur pied dans ces placettes. Il est également intéressant d'extraire un indicateur sur l'ouverture des peuplements matures de hêtraies (surface terrière moyenne des peuplements avec gros bois de Hêtre par exemple).

En plaine, dans son aire de distribution, ce sont les statistiques sur les linéaires de haies qui doivent être analysées. Les placettes de l'inventaire forestier en ripisylve (aulnaie-frênaies notamment) peuvent aussi être analysées, en termes de surface et de gros bois morts sur pied.

\section{DISCUSSION-CONCLUSION}

COMMENT METTRE UNE TELLE SURVEILLANCE EN PLACE ?

Les propositions faites dans la partie précédente nécessitent pour la plupart un travail préalable à partir des données disponibles (SINP et couches de variables environnementales) pour modéliser l'aire de distribution potentielle et définir plus précisément le plan d'échantillonnage (tirer au sort les sites, etc.).

Un accompagnement des gestionnaires, avec des formations, des fiches pratiques et des modèles de tableur pour les variables relatives aux habitats, sera nécessaire.

Avec l'approche "réaliste» volontairement adoptée, qui s'appuie sur la valorisation de dispositifs existants comme les placettes de suivi des habitats forestiers (Benest et al. 2016) ou sur les programmes d'observatoires participatifs, le besoin en production de données spécifiques est maîtrisé. Par ailleurs, les suivis d'habitats peuvent en partie être réa- 
TABLEAU 10. - Synthèse des protocoles recommandés pour une surveillance nationale de Rosalia alpina (Linnaeus, 1758).

\begin{tabular}{|c|c|c|}
\hline Éléments du protocole & Détection - distribution & Suivi d'habitat - population \\
\hline $\begin{array}{l}\text { 1. Paramètres visés par le protocole et } \\
\text { objectifs détaillés }\end{array}$ & $\begin{array}{l}\text { Mieux définir la distribution de l'espèce et } \\
\text { suivre cette distribution dans le temps. }\end{array}$ & $\begin{array}{l}\text { Suivre les surfaces d'habitat potentiel et les } \\
\text { caractéristiques de structure favorable à } \\
\text { l'espèce (chandelles, boisement ouverts). }\end{array}$ \\
\hline $\begin{array}{l}\text { 2. Plan d'échantillonnage national, choix des } \\
\text { sites/placettes }\end{array}$ & $\begin{array}{l}\text { Non structuré. Approche par observatoire } \\
\text { participatif (grand public, professionnels } \\
\text { de la nature, naturalistes) et modélisation } \\
\text { de la distribution potentielle. }\end{array}$ & $\begin{array}{l}\text { Plan d'échantillonnage national de } \\
\text { l'inventaire forestier national (dispositif } \\
\text { placettes IGN: grille de } 1 \times 1 \mathrm{~km} \text {, pour } \\
\text { les points correspondant à des milieux } \\
\text { forestiers). }\end{array}$ \\
\hline 3. Unité d'échantillonnage & France entière & $\begin{array}{l}\text { Placette forestière de l'inventaire forestier } \\
\text { national. Post-stratification pour retenir } \\
\text { les placettes de hêtraie en montage } \\
\text { et de ripisylve en plaine (dans l'aire de } \\
\text { distribution de l'espèce). }\end{array}$ \\
\hline 4. Techniques et matériel & $\begin{array}{l}\text { Remontée des observations par les outils } \\
\text { de saisie en ligne (avec photo) et partage } \\
\text { dans le SINP. }\end{array}$ & $\begin{array}{l}\text { Celui mis en œuvre par l'inventaire forestier } \\
\text { (Benest et al. 2016). }\end{array}$ \\
\hline 5. Période(s) favorable(s) & $\begin{array}{l}\text { Observations d'adultes: mi-juin (plaine) à } \\
\text { mi-août (montagne). }\end{array}$ & Toute l'année. \\
\hline 6. Variables et co-variables à relever & $\begin{array}{l}\text { Photo de l'individu comme preuve } \\
\text { permettant la validation de l'observation. } \\
\text { Inciter à noter l'essence d'arbre et la } \\
\text { situation (exposition, grume ou chandelle, } \\
\text { etc.). }\end{array}$ & $\begin{array}{l}\text { Sélection des variables correspondant à } \\
\text { des traits de l'espèce: gros bois mort sur } \\
\text { pied; ouverture du peuplement (surface } \\
\text { terrière). }\end{array}$ \\
\hline $\begin{array}{l}\text { 7. Technicité/compétence pour effectuer les } \\
\text { relevés }\end{array}$ & $\begin{array}{l}\text { Observation opportuniste. Connaissance } \\
\text { des enquêtes participatives et systèmes } \\
\text { de saisie (enquête Opie, INPN-Espèces, } \\
\text { i-Naturalist pour le public; Carnat, } \\
\text { Géonature pour les naturalistes). }\end{array}$ & Sans objet (porté par l'inventaire forestier). \\
\hline $\begin{array}{l}\text { 8. Possibilité de mobiliser des opérateurs } \\
\text { gestionnaires? }\end{array}$ & $\begin{array}{l}\text { Oui pour le signalement de toutes les } \\
\text { observations dans les bases de données } \\
\text { de leur structure. }\end{array}$ & Sans objet. \\
\hline 9. Périodicité des relevés & Sans objet. & Cycle de 10 ans pour l'inventaire forestier. \\
\hline 10. Type d'indicateur et d'analyse & $\begin{array}{l}\text { Nombre de sites avec observation, nombre } \\
\text { de mailles connues (avec données de } \\
\text { moins de } 10 \text { ans), surface favorable } \\
\text { d'après un modèle de niche. }\end{array}$ & $\begin{array}{l}\text { Densité de gros bois morts dans les } \\
\text { habitats, en distinguant les hêtraies en } \\
\text { montagne et les ripisylves en plaine. } \\
\text { Degré d'ouverture des peuplements }\end{array}$ \\
\hline 11. Éléments de coût & $\begin{array}{l}\text { Animation de programmes participatif } \\
\text { (existent déjà, à maintenir/renforcer). } \\
\text { Traitement et analyse de données } \\
\text { périodiquement (deux ans). }\end{array}$ & $\begin{array}{l}\text { Traitement spécifique des données (tous } \\
\text { les six ans, un an avant les évaluations } \\
\text { DHFF). }\end{array}$ \\
\hline 12. Aspect expérimental à développer & \multicolumn{2}{|c|}{$\begin{array}{l}\text { Recherche sur les populations de plaine de l'Ouest de la France: génétique pour connaître leur } \\
\text { origine et le lien avec les autres populations; niche écologique, densité dans les habitats } \\
\text { de bocage et ripisylve, etc. }\end{array}$} \\
\hline 13. Sites pilotes potentiels & Sans objet. & \\
\hline
\end{tabular}

lisés avec ou par les gestionnaires d'espaces naturels, pour les stations qui sont dans ces espaces. Une des approches possibles serait une petite équipe dédiée à la coordination du suivi de ces espèces (et éventuellement de quelques autres taxons saproxyliques patrimoniaux), à la réalisation des prospections de terrain nécessitant une expertise, à la formation des gestionnaires, à l'animation des acteurs concernés, à la gestion des données et aux premiers niveaux de traitement et d'analyse. Nous estimons qu'une équipe de trois personnes devrait permettre d'assurer cette mission (moyennant des renforts ponctuels, l'appui des réseaux de gestionnaires et de déléguer certaines analyses poussées). Il faut noter un autre facteur favorable: pour plusieurs espèces, il est possible de faire du terrain en dehors du pic d'activité principal de l'entomofaune (mai-juillet), ce qui laisse beaucoup plus de possibilités d'organisation des campagnes de prospection.
Contrairement à la tendance actuelle à la décentralisation des politiques de connaissance de la biodiversité, nous recommandons une approche avec une équipe intervenant sur l'ensemble du territoire, pour une économie d'échelle et pour limiter les sources d'hétérogénéité dans les données. Pour des disciplines très spécialisées comme l'entomologie forestière, cette approche a déjà fait ses preuves (exemple du pôle national d'entomologie forestière de l'ONF basé à Quillan)

\section{OPPORTUNITÉS ET LIMITES DU DÉVELOPPEMENT}

DE LA SURVEILLANCE DES SAPROXYLIQUES DHFF

En allant au-delà du fait que c'est une obligation de la France dans le cadre de ses engagements européens, on peut noter plusieurs opportunités:

- la première est le fait que les sept espèces concernées n'ont pour l'instant fait l'objet de pratiquement aucun effort de suivi à l'échelle des régions biogéographiques. Comparati- 
vement à un même effort financier investi pour des groupes d'espèces déjà en partie suivis, le gain de connaissance serait donc relativement plus fort;

- le nombre limité de spécialistes et l'absence de dispositif préexistant rend également la mise en place plus aisée. Ceci dit, le peu d'experts disponibles explique les lacunes de connaissances actuelles et restera un facteur limitant à l'accélération des découvertes ou des suivis, même en cas de crédits alloués significativement augmentés;

- le rapport coût/espèce est relativement favorable, tout en permettant d'aborder des espèces parapluies pour la conservation de stades particuliers du cycle sylvigénétique.

L'approche structurelle via les "habitats d'espèce» (présence et densité de dendromicrohabitats; diversité et volumes de bois morts : à ne pas confondre avec les « habitats " de nature phytoécologique définis dans l'annexe I de la DHFF) nous semble plus réaliste pour un suivi en routine et moins coûteuse que les approches par dénombrement des individus testées par nos collègues italiens (ex: Campanaro et al. 2017b pour la Rosalie des Alpes). De plus, cette approche «habitats d'espèce" devrait rendre les résultats exploitables pour la gestion conservatoire et faciliter l'appropriation par les acteurs, puisqu'elle s'adresse aux facteurs sur lesquels l'Homme intervient directement.

L'accent mis sur les habitats d'espèces (proxy ou indicateur indirect de présence des espèces) a cependant des limites. Il faut notamment s'assurer du lien fort entre les variables d'habitats et la dynamique des populations d'espèces (indicateurs directs). Déjà en partie démontrée dans la littérature, souvent de manière corrélative, cette approche requiert cependant des recherches approfondies sur quelques sites. Il faudrait notamment une approche expérimentale démontrant l'effet de l'offre d'habitat sur les effectifs.

Par ailleurs, les habitats favorables peuvent être occupés ou pas: ceci nécessite de repréciser que l'on aura affaire dans certains cas à des dettes d'extinction (populations présentes mais habitats de qualité médiocre, en décroissance ou en trop faible quantité) mais également parfois à des crédits de colonisation (habitats favorables plus importants que les populations présentes, voire habitat non encore colonisé).

Notre approche est pensée dans une logique de socle de suivi à l'échelle des régions biogéographiques de la DHFF et donc optimisée par une logique d'échantillonnage. Ceci ne couvre pas tous les besoins de suivi pour la gestion de sites Natura 2000 et des autres espaces protégés. Nous recommandons que les arbres habitats et leur recrutement soient suivis dans l'ensemble des espaces qui abritent ces espèces.

Il faut noter qu'il n'est pas techniquement possible de regrouper dans un plan d'échantillonnage unique toutes ces espèces qui occupent souvent des secteurs différents. Il n'est pas non plus possible de coupler ces suivis d'espèces particulières (par leur rareté et leur biologie) avec des suivis représentatifs des cortèges saproxyliques (où la technique du piège d'interception Polytrap ${ }^{\mathrm{TM}}$ est la technique la plus classique et recommandée [Bouget \& Brustel 2010a, 2010b]). C'est une limitation classique dans le suivi des espèces rares qui s'avère complémentaire d'approches représentatives des cortèges.
En conclusion, les protocoles proposés dans cet article apportent une pierre à l'édifice d'un programme ambitieux de suivi de la biodiversité et font écho à une obligation communautaire et à un besoin sociétal rappelé par exemple dans le plan d'action présenté en 2018 par le ministre en charge de l'écologie.

\section{Remerciements}

La présente étude découle d'une demande de Ministère en charge de l'écologie (direction de l'eau et de la biodiversité) au Muséum national d'Histoire naturel et à l'Agence française pour la Biodiversité afin de développer la surveillance de la biodiversité terrestre. Nous remercions les deux relecteurs anonymes qui ont contribué à améliorer cet article.

\section{RÉFÉRENCES}

Adamski P., Michalcewicz J., Bohdan A. \& Ciach M. 2018. Potential range of impact of an ecological trap network: the case of timber stacks and the Rosalia longicorn. Journal of Insect Conservation 22 (2): 209-219. https://doi.org/10.1007/s10841018-0054-z

Adamski P., Bohdan A., Michalcewicz J., Ciach M., WitKOWSKI Z. 2016. - Timber stacks: potential ecological traps for an endangered saproxylic beetle, the Rosalia longicorn Rosalia alpina. Journal of Insect Conservation 20 (6): 1099-1105. https:// doi.org/10.1007/s10841-016-9932-4

Bardiani M., Chiari S., Maurizi E., Tini M., Toni I., Zauli A., Campanaro A., Carpaneto G.M., Audisio P. 2017. — Guidelines for the monitoring of Lucanus cervus, in CARPANETO G. M., Audisio P., Bologna M. A., Roversi P. F. \& Mason F. (éds), Guidelines for the Monitoring of the Saproxylic Beetles protected in Europe. Nature Conservation 20: 37-78. https://doi. org/10.3897/natureconservation.20.12687

Benest F., Lalanne A., Gauberville C. \& Drapier J. 2016. Premiers éléments pour un dispositif de surveillance de l'état de conservation des habitats forestiers en France. Revue forestière française 118 (5): 409-425.

BensetTiti F. \& Puissauve R. 2015. - Résultats de l'évaluation de l'état de conservation des habitats et des espèces dans le cadre de la directive Habitats-Faune-Flore en France. Rapportage article 17 (Période 2007-2012). SPN-MNHN, Paris, 204 p.

Berger P. 2012. - Coléoptères Cerambycidae de la faune de France continentale et de Corse - Actualisation de l'ouvrage d'André Villiers, 1978. Association Roussillonnaise d'Entomologie, Perpignan, $664 \mathrm{p}$.

BOUGET C. 2004. - Chablis et diversité des coléoptères en forêt feuillue de plaine: impact à court terme de la trouée, de sa surface et de son contexte paysager. Thèse, Cemagref, ENESAD, Muséum national d'Histoire naturelle (Paris), $452 \mathrm{p}$.

Bouget C. \& Brustel H. 2010a. - Chapitre 4: Les groupes d'insectes cibles en forêt tempérée - II Les Coléoptères saproxyliques, in Nageleisen L. M. \& Bouget C. (éds), L'étude des insectes en forêt: méthodes et techniques, éléments essentiels pour une standardisation. Synthèse des réflexions menées par le groupe de travail "Inventaires Entomologiques en Forêt" (Inv.Ent.For). Les Dossiers Forestiers no. 19, Office National des Forêts, 99-110

BOUGET C. \& BRUSTEL H. 2010b. - Chapitre 2: Les pièges vitres, in NAGeleisen L. M. \& Bouget C. (éds), L'étude des insectes en forêt: méthodes et techniques, éléments essentiels pour une standardisation. Synthèse des réflexions menées par le groupe de travail "Inventaires Entomologiques en Forêt" (Inv.Ent.For). Les Dossiers Forestiers no. 19, Office National des Forêts, 58-62. 
Braud Y., Brustel H., Valladares L. \& Cuvelier J. 2016. Bilan des connaissances, inventaire et cartographie de deux coléoptères d'intérêt communautaire (Stephanopachys linearis et S. substriatus) dans les sites Natura 2000 de la région PACA. Résultats 2015. Rapport ENTOMIA pour la DREAL PACA, ENTOMIA, Thèze, 40 p. [non publié]

Braud Y., Brustel H., Valladares L., Bence S., Gouix N. \& Cuvelier J. 2017. - Bilan des connaissances, inventaires 2016 et cartographie de cinq coléoptères saproxyliques d'intérêt communautaire dans les sites Natura 2000 de la région PACA. Stephanopachys linearis, Stephanopachys substriatus, Limoniscus violaceus, Osmoderma eremita et Rosalia alpina. Rapport ENTOMIA pour la DREAL PACA. ENTOMIA, Thèze, 39 p.

BRUSTEL H. 2002 - Coléoptères saproxyliques et valeur biologique des forêts françaises. Perspectives pour la conservation du patrimoine naturel. Thèse de Doctorat de l'Institut National Polytechnique de Toulouse (Spécialité: Sciences Agronomiques), 327 p.

Brustel H. \& Gouix N. 2011. - Coléoptères Rhysodidae en France: données complémentaires pour Rhysodes sulcatus (F., 1787) et incitation à la recherche d'Omoglymmius (s. s.) germari (Ganglbauer, 1892). L'Entomologiste 67 (6): 321-325.

BRUSTEL H. \& Gouix N. 2012. — La chasse aux mythes! Petite contribution cryptoentomologique sur les coléoptères de la Directive Habitats à rechercher en France. Le Coléoptériste 15 (1): 26-37.

Brustel H., Gouix N., Bouyon H. \& Rogé J. 2013. - Les Stephanopachys spp. de la faune ouest-paléarctique (Coleoptera, Bostrichidae). Distribution et reconnaissance des trois espèces françaises au service de l'application de la Directive Habitats. L'Entomologiste 69 (1): 41-50.

BURAKOWSKI B. 1975. - Descriptions of larva and pupa of Rhysodes sulcatus (F.) (Coleoptera, Rhysodidae) and notes on the bionomy of this species. Annales zoologici 32 (12): 271-287.

BUSE J., SCHRÖDER B. \& ASSMANN T. 2007. — Modelling habitat and spatial distribution of an endangered longhorn beetle - A case study for saproxylic insect conservation. Biological Conservation 137: 372-381. https://doi.org/10.1016/j.biocon.2007.02.025

Buse J., RANiUs T. \& ASSMANN T. 2008. - An endangered longhorn beetle associated with old oaks and its possible role as an ecosystem engineer. Conservation Biology 22 (2): 329-337. https:// doi.org/10.1111/j.1523-1739.2007.00880.x

Campanaro A., Hardersen S., Sabbatini Peverieri G. \& CarPANETO G. M. (ÉDS) 2017a. - Monitoring of saproxylic beetles and other insects protected in the European Union. Nature Conservation 19: 1-243.

Campanaro A., Redolfi De Zan L., Hardersen S., Antonini G., Chiari S., Cini A., Mancini E., Mosconi F., Rossi de Gasperis S., Solano E., Bologna M. A. \& Sabbatini Peverieri G. 2017b. - Guidelines for the monitoring of Rosalia alpina, in Carpaneto G. M., Audisio P., Bologna M. A., Roversi P. F. \& MASON F. (éds), Guidelines for the Monitoring of the Saproxylic Beetles protected in Europe. Nature Conservation 20: 165-203. https://doi.org/10.3897/natureconservation.20.12728

CARDOSO P. 2012. - Habitats Directive species lists: urgent need of revision. Insect Conservation and Diversity 5: 169-174. https:// doi.org/10.1111/j.1752-4598.2011.00140.x

Carpaneto G. M., Audisio P., Bologna M.A., Roversi P. F. \& MASON F. (éds) 2017. — Guidelines for the Monitoring of the Saproxylic Beetles protected in Europe. Nature Conservation 20: 1-297.

CAstro A. \& Fernández J. 2016. - Tree selection by the endangered beetle Rosalia alpina in a lapsed pollard beech forest. Journal of insect conservation 20 (2): 201-214. https://doi.org/10.1007/ s10841-016-9854-1

Casula P. 2017. - Monitoring and management of Cerambyx cerdo in the Mediterranean region - a review and the potential role of citizen science, in Campanaro A., Hardersen S., Sabbatini Peverieri G. \& Carpaneto G. M. (éds.), Monitoring of saproxylic beetles and other insects protected in the European
Union. Nature Conservation 19: 97-110. https://doi.org/10.3897/ natureconservation.19.12637

Cateau E., Larrieu L., Vallauri D., Savoie J.-M., Touroult J. \& BRUSTel H. 2015. - Ancienneté et maturité: deux qualités complémentaires d'un écosystème forestier. Comptes Rendus Biologies 338: 58-73. https://doi.org/10.1016/j.crvi.2014.10.004

Chiari S., Carpaneto G. M., Zauli A., Zirpoli G. M., Audisio P. \& RANIUS T. 2013a. - Dispersal patterns of a saproxylic beetle, Osmoderma eremita, in Mediterranean woodlands. Insect conservation and Diversity 6 (3): 309-318. https://doi.org/10.1111/j.17524598.2012.00215.x

Chiari T., Zauli A., Mazziotta A., Luiselli L., Audisio P. \& Carpaneto G. M. 2013b. - Surveying an endangered saproxylic beetle, Osmoderma eremita, in Mediterranean woodlands: a comparison between different capture methods. Journal of Insect Conservation 17 (1): 171-181.

CizeK L., SCHLAghamerskÝ J., BoŘUCKÝ J., HaUCK D. \& Helešic J. 2009. - Range expansion of an endangered beetle: Alpine Longhorn Rosalia alpina (Coleoptera: Cerambycidae) spreads to the lowlands of Central Europe. Entomologica Fennica 20 (3): 200-206.

DG ENVIRONMENT 2017. - Reporting under Article 17 of the Habitats Directive: Explanatory notes and guidelines for the period 2013-2018. Brussels, 188 p. http://cdr.eionet.europa.eu/help/ habitats_art17

DÖRING E. 1955. - Zur Biologie des grossen Eichenbockkäfers (Cerambyx cerdo L.) unter besonderer Berücksichtigung der Populationsbewegungen im Areal. Zeitschrift Angewandte Zoologie 42: 251-373.

Drag L., Hauck D., PoKluda P., Zimmermann K. \& Cizek L. 2011. - Demography and dispersal ability of a threatened saproxylic beetle: a mark-recapture study of the Rosalia Longicorn (Rosalia alpina). PLoS One 6 (6): e21345. https://doi. org/10.1371/journal.pone.0021345

Drumont A., Cammaerts R., Van Nuffels C. \& Navez P. 2012. - Cerambyx cerdo Linnaeus 1758 en Belgique (Coleoptera, Cerambycidae). Lambillionea 112: 61-73.

DuBOIS G. 2009. - Ecologie des coléoptères saproxyliques: Biologie des populations et conservation d'Osmoderma eremita (Coleoptera: Cetoniidae). Thèse de l'Université de Rennes 1, École doctorale Vie-Agro-Santé, 218 p.

EHNSTRÖM B. \& AXELSSON R. 2002. - Insektsgnag i bar koch ved. Uppsala, ArtDatabanken, SLU, 130 p.

EVANS D. \& ARVELA M. 2011. - Assessment and reporting under Article 17 of the Habitats Directive: Explanatory Notes \& Guidelines for the period 2007 - 2012. European Topic Centre on Biological Diversity, 123 p.

Flores M. DE \& Sueur A. 2015. — La Rosalie des Alpes. Premiers résultats d'enquête. Insectes 176 (1): 37-38.

Fuchs L., Callot H., Godinat G. \& BRustel H. 2014. — Cucujus cinnaberinus (Scopoli, 1763), nouvelle espèce pour la faune de France (Coleoptera Cucujidae). L'Entomologiste 70 (4): 279-287.

GouIX N. 2011. - Gestion forestière et biodiversité, les enjeux de conservation d'une espèce parapluie: Limoniscus violaceus (Coleoptera). Thèse de doctorat de l'université Pierre et Marie Curie, École Doctorale de la Diversité du Vivant, Paris, 258 p.

GouiX N. \& BRUSTEL H. 2012. - Emergence trap, a new method to survey Limoniscus violaceus (Coleoptera: Elateridae) from hollow trees. Biodiversity and Conservation 21: 421-436. https://doi. org/10.1007/s10531-011-0190-1

GouiX N. \& VAlladares L. 2015. — Prise en compte des coléoptères dans le réseau Natura 2000 en Midi-Pyrénées. Rapport DREAL, Toulouse, $49 \mathrm{p}$.

Gouix N., Mertlik J., Jarzabek-Muller A., Nemeth T. \& BRUSTEL H. 2012. - Known status of the endangered western Palaearctic violet click beetle (Limoniscus violaceus) (Coleoptera). Journal of Natural History 46: 796-802. https://doi.org/10.1080 /00222933.2011.651639

Gouix N., Sebek P., Valladares L., Brustel H. \& Brin A. 
2015. - Habitat requirements of the violet click beetle (Limoniscus violaceus), an endangered umbrella species of basal hollow trees. Insect Conservation and Diversity 8 (5): 418-427. https:// doi.org/10.1111/icad.12119

Harvey D. J., Gange A. C., Hawes C. J. \& Rink M. 2011. Bionomics and distribution of the stag beetle, Lucanus cervus (L.) across Europe. Insect Conservation and Diversity 4 (1): 23-38. https://doi.org/10.1111/j.1752-4598.2010.00107.x

HEDIN J. 2003. - Metapopulation ecology of Osmoderma eremitadispersal, habitat quality and habitat history. Thesis, Department of Zoology. Lund University, Sweden, 138 p.

Houard X., Mériguet B. \& Merlet F. 2013. - Enquête Lucane. Jamais deux sans trois... Insectes 169 (2): 33-34.

Hyvärinen E., Kouki J., Martikainen P. 2006. — Fire and green-tree retention in conservation of red-listed and rare deadwood-dependent beetles in finnish boreal forests. Conservation Biology 20(6): 1711-1719. https://doi.org/10.1111/j.15231739.2006.00511.x

IChter J., Poncet L. \& Touroult J. 2014. - Catalogues des méthodes et des protocoles. Phase 1: Étude de définition et proposition d'une démarche. Service du patrimoine naturel, Muséum national d'Histoire naturelle, Paris. SPN 2014-52, 30 p.

Kostanjsek F., Sebek P., Baranova B., Seric Jelaska L., Riedi V. \& CizeK L. 2018. - Size matters! Habitat preferences of the wrinkled bark beetle, Rhysodes sulcatus, the relict species of European primeval forests. Insect Conservation and Diversity 11 (6): 545-553. https://doi.org/10.1111/icad.12295

Lachat T., Ecker K., Duelli P. \& Wermelinger B. 2013. Population trends of Rosalia alpina (L.) in Switzerland: a lasting turnaround? Journal of Insect Conservation 17: 653-662. https:// doi.org/10.1007/s10841-013-9549-9

LARRIEU L. 2014. - Les dendro-microhabitats: facteurs clés de leur occurrence dans les peuplements forestiers, impact de la gestion et relations avec la biodiversité taxonomique. Doctorat de l'Université de Toulouse, 333 p. + annexes

LARRIEU L. \& GONIN P. 2008. — L'indice de biodiversité potentielle (IBP) : une méthode simple et rapide pour évaluer la biodiversité potentielle des peuplements forestiers. Revue forestière française 110: 727-748.

Larrieu L., Paillet Y., Winter S., Bütler R., Kraus D., Krumm F. Lachat T., Michel A.K., Régnery B. \& VANDEKerKhove K. 2018. - Tree related microhabitats in temperate and Mediterranean European forests: A hierarchical typology for inventory standardization. Ecolological Indicators 84: 194-207. https://doi. org/10.1016/j.ecolind.2017.08.051

LESEIGNEUR L. 1951. - Capture de deux coléoptères rares, à Abriès (H.A.). L'Entomologiste 7 (2-3): 87.

LESNE P. 1904. - Synopsis des Brostrychides paléarctiques. L'Abeille 30: $153-168$.

LESNE P. 1909. - Communication: Stephanopachys substriatus Paykull, Bostrychide nouveau pour la faune française. Bulletin de la Société entomologique de France, 102-103.

LuCE J. M. 1996. - Limoniscus violaceus (Müller P.W., 1821), in Helsdingen P. J., Willems L. \& Speight M. C. D. (éds.), Background Information on Invertebrates of the Habitats Directive and the Bern Convention. Part I - Crustacea, Coleoptera and Lepidoptera, Nature and Environment. Council of Europe, Strasbourg: 49-52.

MichalCEWICZ J. \& CiACH M. 2012. - Rosalia longicorn Rosalia alpina (L.) (Coleoptera: Cerambycidae) uses roadside European ash trees Fraxinus excelsior L. - an unexpected habitat of an endangered species. Polish Journal of Entomology/Polskie Pismo Entomologiczne 81 (1): 49-56.

Michalcewicz J., Ciach M. \& BodziarczyK J. 2011. - The unknown natural habitat of Rosalia alpina (L.) (Coleoptera: Cerambycidae) and its trophic association with the mountain elm Ulmus glabra in Poland-a change of habitat and host plant. Polish Journal of Entomology/Polskie Pismo Entomologiczne 80 (1): 23-31.
Müller J., JarzabeK-Müller A. \& Bussler H. 2013. — Some of the rarest European saproxylic beetles are common in the wilderness of Northern Mongolia. Journal of Insect Conservation 17 (5): 989-1001. https://doi.org/10.1007/s10841-013-9581-9

Noblecourt T. 2005. - Recommandations sylvicoles pour la conservation de Rosalia alpina (Linné) (Insecta, Coleoptera, Cerambycidae). Les Rendez-vous techniques de l'ONF 9: 46-49.

PAILlET Y. 2017. - Suivis nationaux de biodiversité en forêt en France: une lecture au travers des variables essentielles de Biodiversité. Naturae 2017 (6): 1-11.

Puissauve R., GaZay C. \& Touroult J. 2017. — Suivi et surveillance des espèces «protégées» (Directives, code environnement...), in Touroult J., Chaumet S., Poncet L. \& Siblet J.-P. (éds), Diagnostic et recommandations pour une stratégie d'acquisition de connaissances naturalistes continentales. Tome I: Analyse des besoins et des dispositifs existants. Rapport MNHN-SPN/UMS2006-PatriNat, $n^{\circ}$ 2017-10, Paris: 137-149.

Rabinovitch A., Flores M. De \& Houard X. 2017. — Lucane et Rosalie, l'enquête avance. Insectes 185 (2): 29-30.

Ranius T. 2002. - Osmoderma eremita as an indicator of species richness of beetles in tree hallows. Biodiversity and conservation 11: 931-941.

Ranius T. \& Nilsson S. G. 1997. — Habitat of Osmoderma eremita Scop. (Coleoptera: Scarabaeidae), a beetle living in hollow trees. Journal of Insect Conservation 1: 193-204

RANiUS T. \& HEDIN J. 2001. - The dispersal rate of a beetle, Osmoderma eremita, living in tree hollows. Oecologia 126 (3) 363-370. https://doi.org/10.1007/s004420000529

Ranius T., NikLasson M. \& BERG N. 2009. - Development of tree hollows in pedunculate oak (Quercus robur). Forest Ecology and management 257 (1): 303-310. https://doi.org/10.1016/j. foreco.2008.09.007

Ranius T., Aguado L. O., ANTONSSON K., Audisio P., Ballerio A., Carpaneto G. M., Chobot K., Guurašin B., Hanssen O., Huijbregts H., Lakatos F., Martin O., Neculiseanu Z., Nikitsky N. B., Paill W., Pirnat A., Rizun V., Ruicănescu A., Stegner J., Süda I., Szwalko P., Tamutis V., Telnov D., TSINKEVICH V., VERSTEIRT V., VignON V., VÖGELI M. \& ZACH P. 2005. - Osmoderma eremita (Coleoptera, Scarabaeidae, Cetoniinae) in Europe. Animal biodiversity and conservation 28 (1): 1-44.

REDOlFi De ZAN L., BARDiani M., ANTONINI G., CAMPANARO A., Chiari S., Mancini E., Maura M., Sabatelli S., Solano E., Zauli A., Peverieri G. S. \& Roversi P.F. 2017. - Guidelines for the monitoring of Cerambyx cerdo. Nature Conservation 20: 129-164.

Rudolph V. K. 1974. — Beitrag zur Kenntnis der Elateridenlarven der Fauna der DDR und der BRD. Zoologische Jahrbücher: Abteilung für Systematik, Okologie und Geographie der Tiere 101: 1-151.

Russo D., Cistrone L. \& GARONNA A. P. 2011. - Habitat selection by the highly endangered long-horned beetle Rosalia alpina in Southern Europe: a multiple spatial scale assessment. Journal of Insect Conservation 15 (5): 685-693.

SAMA G. 2002. - Atlas of the Cerambycidae of Europe and the Mediterranean Area. Volume 1: Northern, Western, Central and Eastern Europe, British Isles and Continental Europe from France (excl. Corsica) to Scandinavia and Urals. Nakladatelstvi Kabourek, Zlin, $173 \mathrm{p}$.

SimON A. 1952. - Trois coléoptères rares dans la vallée du Queyras (05). L'Entomologiste 8 (2): 64.

SpeIGHT M. C. D. 1989. - Les invertébrés saproxyliques et leur protection. Collection Sauvegarde de la nature, $\mathrm{N}^{\circ} 42$, Conseil de l'Europe, Strasbourg, $77 \mathrm{p}$.

Touroult J., Chaumet S., Poncet L. \& Siblet J.-P. (éds) 2017. Diagnostic et recommandations pour une stratégie d'acquisition de connaissances naturalistes continentales. Tome II: Synthèse et propositions d'actions. Rapport MNHN-SPN/UMS-2006-PatriNat, $\mathrm{n}^{\circ}$ 2017-11, $79 \mathrm{p}$.

VAlainis U., Nitcis M., AKsJuta K., BARŠEVsKis A., Cibul,Skis R., 
Balalaikins M., Avgin S. S. 2015. - Results of using pheromone-baited traps for investigations of Osmoderma barnabita Motschulsky, 1845 (Coleoptera: Scarabaeidae: Cetoniinae) in Latvia. Baltic Journal of Coleopterology 15 (1): 37-45.

VignON V. 2005. — Le rôle des habitats de substitution (bocage, parcs, arbres d'alignement) pour la survie des espèces cavicoles et saproxyliques - Implications et synergie avec la conservation en milieu forestier: 293-300, in VALLAURi D., ANDrÉ J., Dodelin B., Eymard-Machet R. \& Rambaud D. (éds.), Bois mort et à cavités: une clé pour des forêts vivantes. Lavoisier Tec et Doc, Paris: 293-300.

VignON V. 2006. - Le pique-prune, histoire d'une sauvegarde. Catiche production, Nohanent, O.G.E. - Cofiroute Ed., 32 p.

VignON V. 2014. - Le Pique-Prune: une histoire liée à celle des vieux arbres à cavités. Le Courrier de la Nature 285: 27-33.

Vignon V. 2017. — Osmoderma eremita (Scopoli, 1763), le Piqueprune, et la construction de l'autoroute A28 dans la Sarthe et dans l'Orne: une prise en compte riche d'enseignements, in GOUIX N. \& MARC D. (éds), Les invertébrés dans la conservation et la gestion des espaces naturels. Actes du colloque de Toulouse du 13 au 16 mai 2015. Muséum national d'Histoire naturelle, Paris. (Patrimoine naturel ; 76): 65-69.

VignON V. \& ORABI P. 2002. — Note de synthèse sur l'inventaire aérien des habitats de l'Osmoderma eremita dans un secteur du département de l'Aveyron, in BRUSTEL H. (éd.), Inventaire d'Osmoderma eremita en Aveyron. (Commande DIREN Midi-Pyrénées, 5 février 2002). Document de synthèse, ESAP, 18 p. + Annexes

Vignon V. \& ORABI P. 2003a. — Une recherche par avion pour trouver les sites de conservation des insectes du bocage. Le Courrier de la Nature 205: 32-35.

VigNON V. \& ORABI P. 2003b. - Exploring the hedgerows network in the west of France for the conservation of saproxylic beetles (Osmoderma eremita, Gnorimus variabilis, Lucanus cervus, Cerambyx cerdo): 36-38, in BOWEN C. P. (éd.), Proceedings of the Second pan-European Conference on Saproxylic Beetles. People's trust for Endangered species, London, $77 \mathrm{p}$.

VILliers A. 1978. - Faune des Coléoptères de France I-Cerambycidae. Encyclopédie Entomologique - XLII. Editions Lechevalier, Paris, $611 \mathrm{p}$

Zapponi L., Cini A., Bardiani M., Hardersen S., Maura M., Maurizi E., Redolfi De Zan L., Audisio P., Bologna M. A., Carpaneto G. M., Roversi P. F., Sabbatini Peverieri G., Mason F. \& CAmpanaro A. 2017. - Citizen science data as an efficient tool for mapping protected saproxylic beetles. Biological Conservation 208: 139-145. https://doi.org/10.1016/j. biocon.2016.04.035

Soumis le 12 février 2019; accepté le 7 juin 2019 publié le 10 septembre 2019. 